\title{
WestVirginiaUniversity
}

THE RESEARCH REPOSITORY @ WVU

Graduate Theses, Dissertations, and Problem Reports

2005

\section{Functional MRI of rat kidney using BOLD \& ASL techniques}

\author{
Rajesh K. Garugu \\ West Virginia University
}

Follow this and additional works at: https://researchrepository.wvu.edu/etd

\section{Recommended Citation}

Garugu, Rajesh K., "Functional MRI of rat kidney using BOLD \& ASL techniques" (2005). Graduate Theses, Dissertations, and Problem Reports. 2233.

https://researchrepository.wvu.edu/etd/2233

This Thesis is protected by copyright and/or related rights. It has been brought to you by the The Research Repository @ WVU with permission from the rights-holder(s). You are free to use this Thesis in any way that is permitted by the copyright and related rights legislation that applies to your use. For other uses you must obtain permission from the rights-holder(s) directly, unless additional rights are indicated by a Creative Commons license in the record and/ or on the work itself. This Thesis has been accepted for inclusion in WVU Graduate Theses, Dissertations, and Problem Reports collection by an authorized administrator of The Research Repository @ WVU. For more information, please contact researchrepository@mail.wvu.edu. 
Functional MRI of Rat Kidney using BOLD \& ASL Techniques

\author{
Rajesh K. Garugu
}

Thesis submitted to the

College of Engineering and Mineral Resources

at West Virginia University

in partial fulfillment of the requirements

for the degree of

Master of Science

in

Electrical Engineering

Dr. Susan Lemieux, Ph.D., Co-Chair

Dr. L. A. Hornak, Ph. D., Chair

Dr. Xin Li, Ph.D.

Lane Department of Computer Science and Electrical Engineering

Center for Advanced Imaging, WVU School of Medicine

\author{
Morgantown, West Virginia \\ 2005
}

\begin{abstract}
Keywords:
Magnetic Resonance Imaging, Blood Oxygenation Level Dependent, Arterial Spin Labelling, laser Doppler flowmetry, fluorescence quenching, perfusion imaging, tissue oxygenation, blood flow.
\end{abstract}




\section{ABSTRACT \\ Functional MRI of Rat Kidney Using BOLD \& ASL Techniques}

\section{Rajesh K Garugu}

Noninvasive functional magnetic resonance imaging (fMRI) methods may provide new ways to detect and track renal hemodynamic changes in-vivo. In this thesis, two fMRI methods were correlated with simultaneous invasive hemodynamic measurements. A particular goal of this thesis was to measure the relative contributions of oxygenation and perfusion changes to changes in the relaxation rate, $\mathrm{T}_{2} *$. Also, an MRI compatible motion detector was built and along with the invasive probes was interfaced to a data acquisition system for use during scanning. Drug-induced changes in renal oxygenation and blood flow were measured by BOLD- \& ASL-MRI noninvasively, while a dual oxygenation/perfusion optical-probe was used for the invasive measurements. Six sets of results were obtained. Values of $\mathrm{T}_{2}{ }^{*}, \mathrm{LDF}$ and $\mathrm{pO}_{2}$ correlated in four of the data sets while the other two were discrepant. BOLD images were of high quality while ASL perfusion maps were of inadequate spatial resolution and poor quality. 


\section{ACKNOWLEDGEMENTS}

I first express my sincere thanks to Dr. Susan Lemieux for providing me an opportunity to work with her, and for being my advisor and friend. Her guidance and comments have guided me all through my college career. I would like to thank Dr. Aina Puce and Dr. Ray Raylman, for taking special interest in this project and also for providing valuable comments.

I put forward my gratitude to my committee members, Dr. L. A. Hornak and Dr. Xin $\mathrm{Li}$, for their support and contribution in the course of this study.

I gratefully acknowledge the cooperation and support of Dr. Chris Baylis and Dr. Mary Davis from the Department of Pharmacology and Physiology, WVU. I would also like to thank Mr. Kevin Engels for all his help with the experiments and data acquisition.

Also, I would like to thank the members of Center for Advanced Imaging / Department of Radiology for all their support and guidance.

I thank all my family members, relatives and friends for their well wishes and continuing support. 


\section{DEDICATIONS}

I am honored to dedicate this publication to the members of my family who have encouraged me and guided me during my career. I express my love and gratefulness to my parents, Narayana Rao and Naga Lakshmi, and my sisters Sunitha and Virtha, who have inspired and guided me through out my life. Without their endless efforts, I could never have fulfilled my dream of pursuing my MS.

Also, I would like to thank all my friends who have helped me directly or indirectly at every stage of my life. 


\section{TABLE OF CONTENTS}

Title page

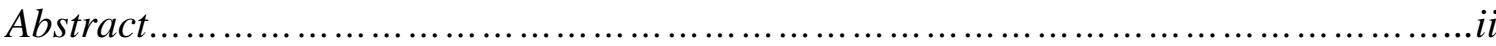

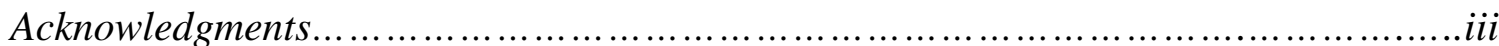

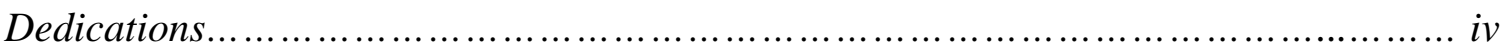

Table of Contents..............................................................................

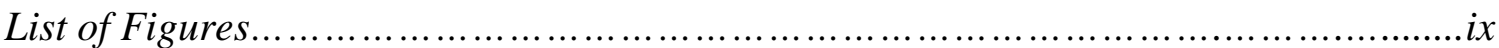

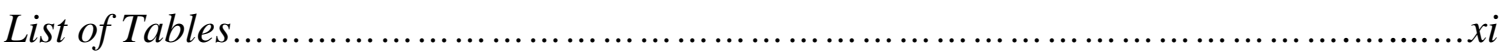

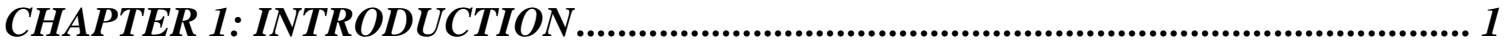

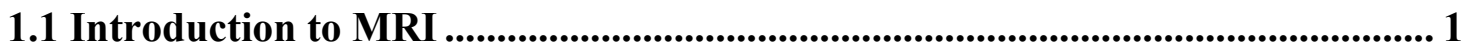

1.2 Motivation and Objective of this thesis..................................................................... 2

1.3 Functional Anatomy of the Kidney .................................................................. 3

1.3.1 Renal Blood Flow and Oxygenation............................................................ 4

1.4 Potential Application of this study .............................................................. 5

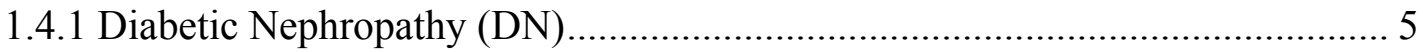

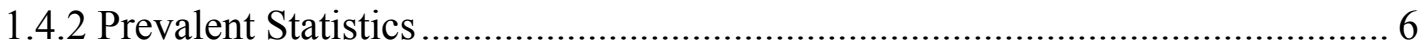

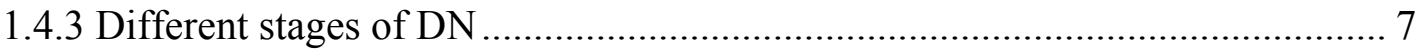

1.4.4 Need for Early Detection and Diagnosis .................................................. 7

CHAPTER 2: METHODS AND DETAILS ........................................................... 8

2.1 Blood Oxygenation and Level Dependent (BOLD) MRI .................................. 8

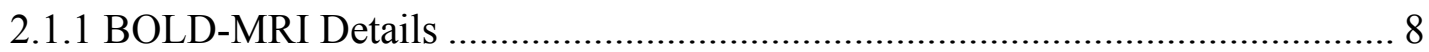

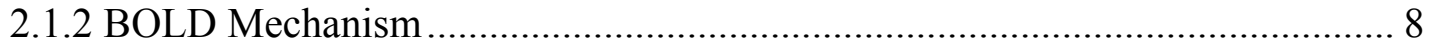

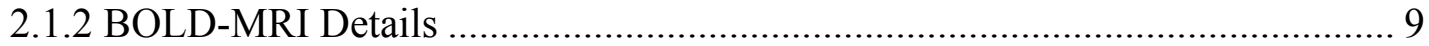

2.2 Arterial Spin Labeling ............................................................................ 11

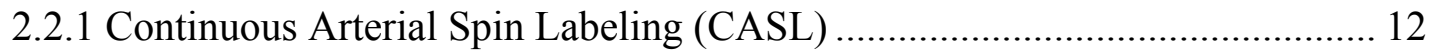

2.2.2 Pulsed Arterial Spin Labeling (PASL) .................................................... 13 


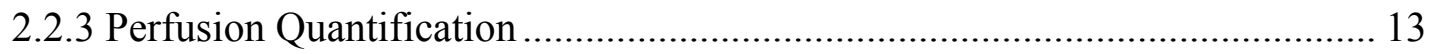

2.2.4 Flow-sensitive Alternating Inversion Recovery (FAIR)................................. 15

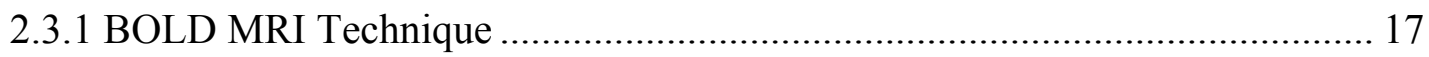

2.3.2 Arterial Spin Labeling (ASL) MRI Technique ............................................. 18

2.3.3 Validation of MRI with Invasive Techniques.................................................. 19

2.4 Approach to the problem ........................................................................................... 19

2.4.1 Optical Probe Oxygenation and Blood Flow Measurement Methods ............. 20

2.4.2 Fluorescence Quenching Oximetry............................................................. 20

2.4.3 Laser Doppler Flowmetry (LDF) ............................................................ 21

CHAPTER 3: EXPERIMENTAL DESIGN AND SPECIFICATIONS......................... 23

3.1 Technical Specifications ............................................................................................. 23

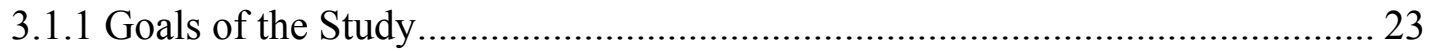

3.1.2 MR Imaging Pulse Sequences ................................................................ 24

3.1.3 Fast Spin Echo (FSE) Imaging ................................................................. 24

3.1.4 Spoiled Gradient Echo (SPGR) …………………….............................. 25

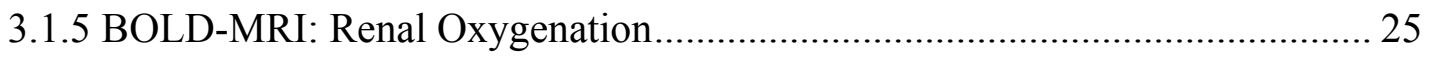

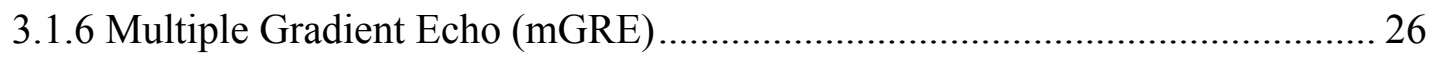

3.1.7 Perfusion MRI using Arterial Spin Labeling .................................................. 27

3.1.8 Dual Probe OxyLab/OxyFlo System …………………………………....... 27

3.1.9 OxyLab Fluorescence Quenching Oximetry ................................................. 28

3.1.10 OxyLab Laser Doppler Flowmetry .............................................................. 29

3.1.11 MR Compatible Motion Detector ................................................................. 31

3.2 MRI \& Probe Experimental Design \& Procedures .................................................. 33

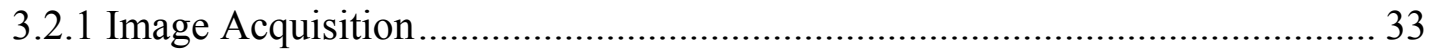

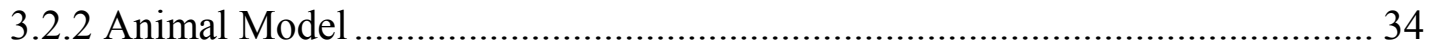

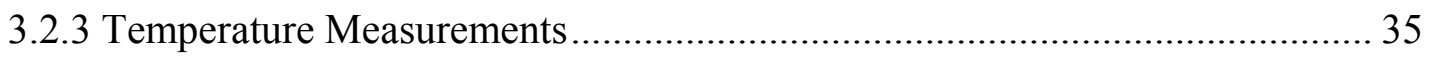

3.2.4 Animal Preparation \& Experimental Set-up ................................................... 36

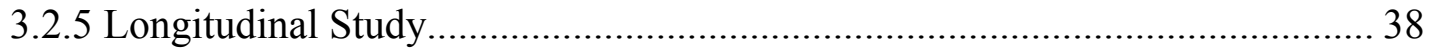

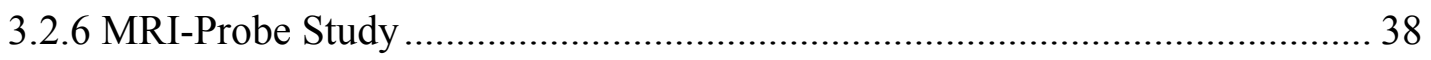

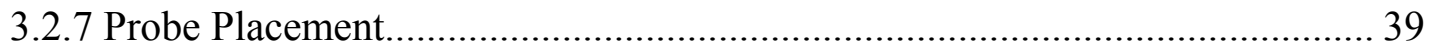




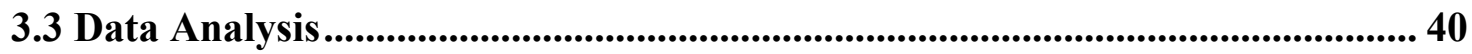

3.3.1 MRI-Oxygenation Data Analysis ................................................................ 40

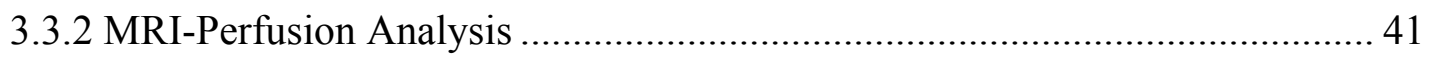

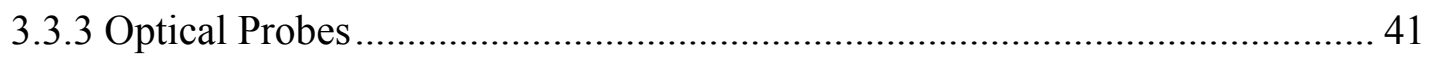

3.3.4 Comparing the Invasive and Noninvasive Methods ....................................... 42

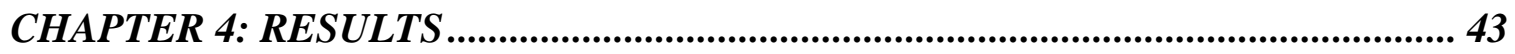

4.1 Physiological Monitoring.............................................................................................. 43

4.1.1 Temperature Measurements............................................................................. 43

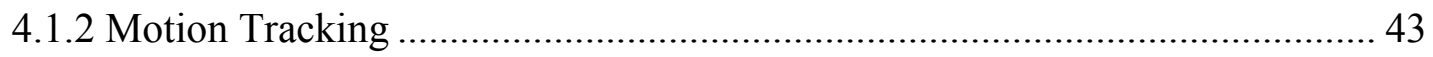

4.2 Longitudinal Study ................................................................................................. 45

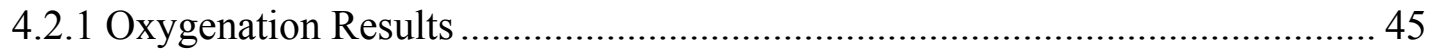

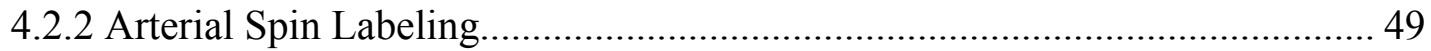

4.3 MRI-Probe Study ............................................................................................................ 53

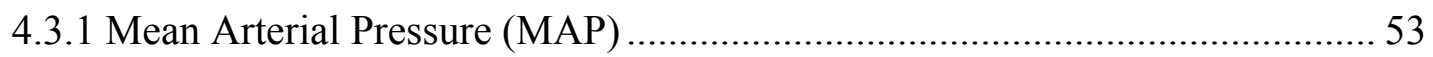

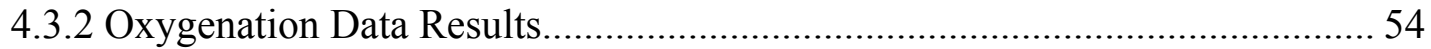

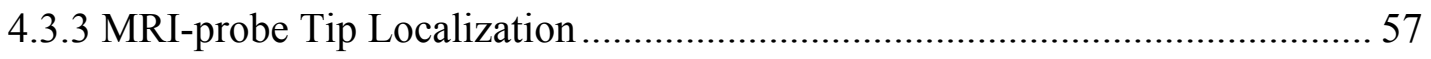

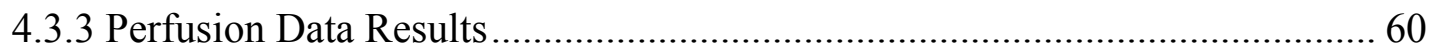

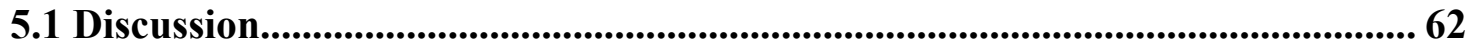

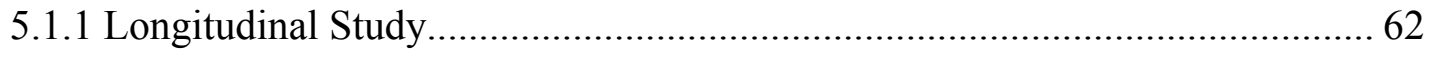

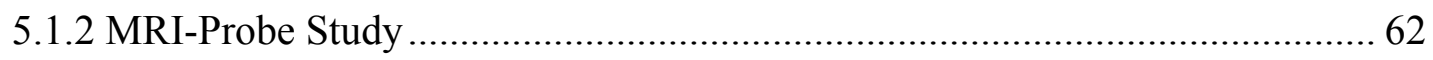

5.2 Conclusion ............................................................................................................................... 64

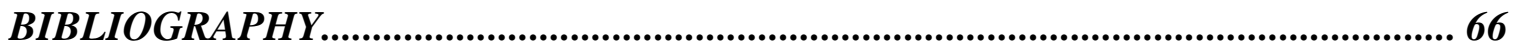

APPENDIX ..................................................................................................................... 69

A.1 Fundamentals of MRI................................................................................................ 69

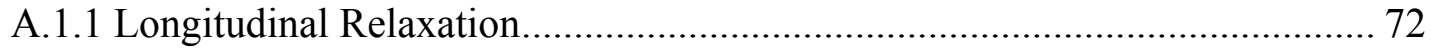

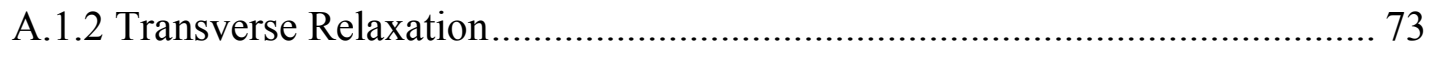

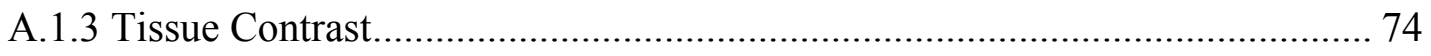

A.2 Localization of the MR signal .................................................................................. 74 


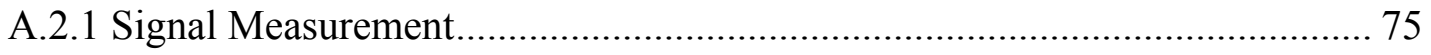

A.2.2 Signal Localization using Gradients ........................................................ 76

A.3 MR Pulse Sequences ................................................................................ 77

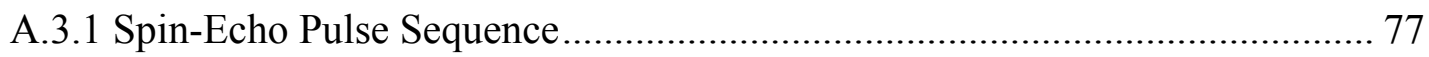

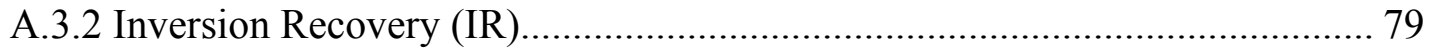

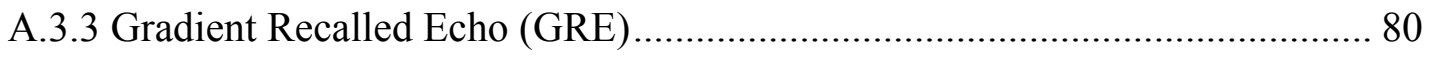

$* * *$ 


\section{LIST OF FIGURES}

Figure 1.1: Kidney anatomy and functioning.......................................................... 3

Figure 1.2: Plot indicating kidney failure over time in $D N$. ....................................... 7

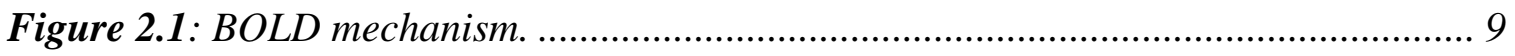

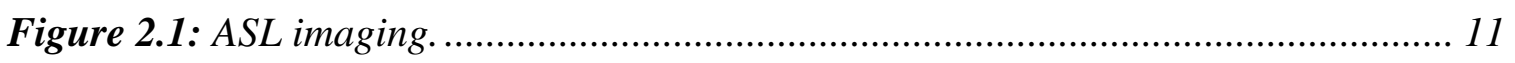

Figure 2.2: Continuous tagging and simultaneous acquisition. ................................... 12

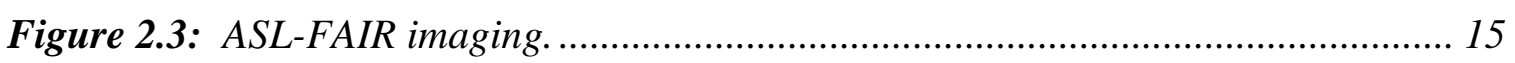

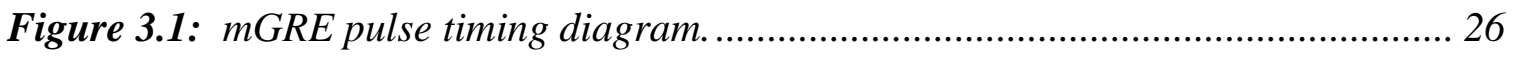

Figure 3.2: Plot between $\ln (\mathrm{SI})$ and echo time indicating the $T_{2}{ }^{*}$ decay....................... 26

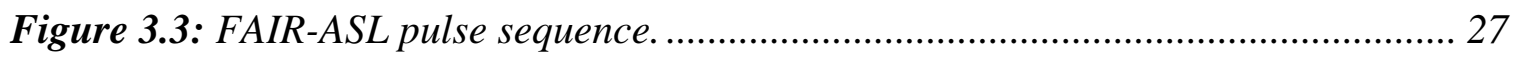

Figure 3.5 Set up for real-time invasive oxygenation and perfusion measurements. ...... 28

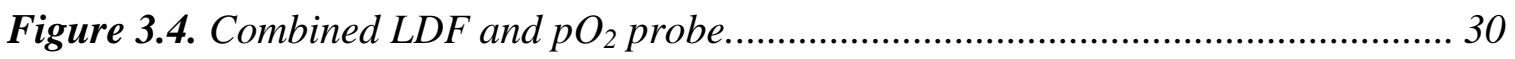

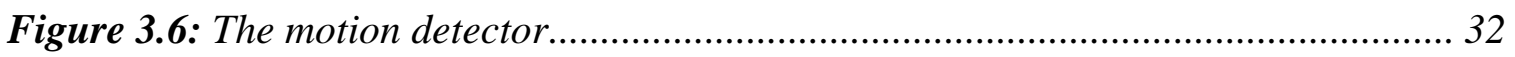

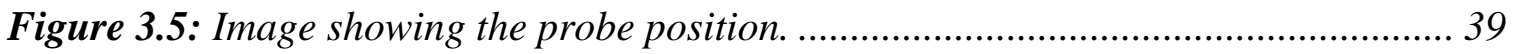

Figure 4.1: Plot indicating variation of animal body temperature with time................. 43

Figure 4.2: MacLab plot indicating animal movement............................................... 44

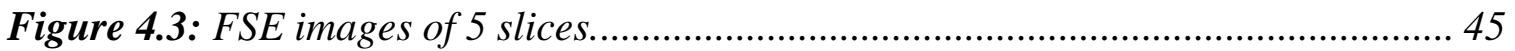

Figure 4.4: Understanding MRI-FSE image ......................................................... 45

Figure 4.5: mGRE image set with varying TE. .................................................... 46

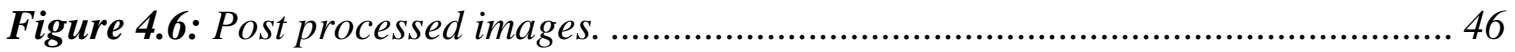

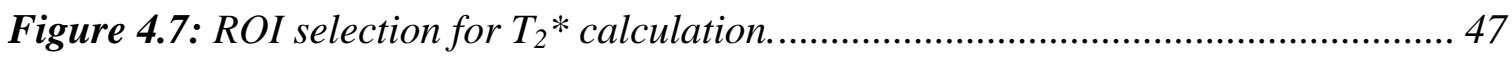

Figure 4.8: Plot indicating the changes in posterior cortex $T_{2}{ }^{*}$ with respect to time.... 48

Figure 4.9: Plot indicating the changes in anterior cortex $T_{2}{ }^{*}$ with respect to time....... 48

Figure 4.10: Plot indicating the changes in medulla $T_{2}{ }^{*}$ with respect to time............... 49

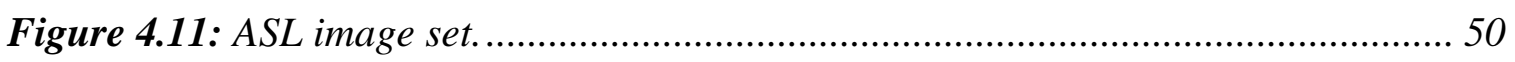

Figure 4.12: Tagged and control images of all 5 slices.............................................. 50

Figure 4.13: Perfusion weighted images obtained using Matlab processing ................. 51

Figure 4.14: ROI selection for perfusion calculations.............................................. 51

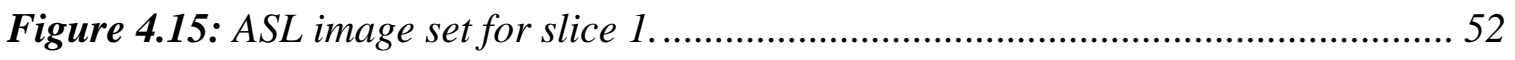

Figure 4.16: Plot indicating changes in MAP with respect to the three time periods..... 54

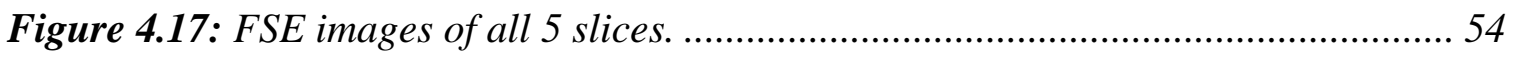




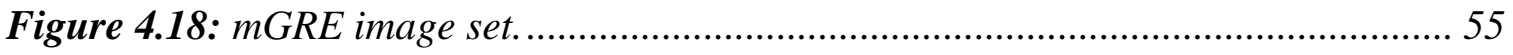

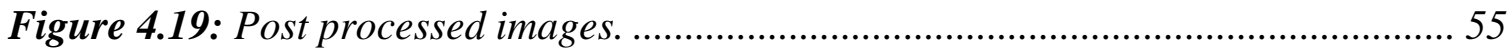

Figure 4.20: ROI selection for $T_{2}{ }^{*}$ calculations. .................................................... 55

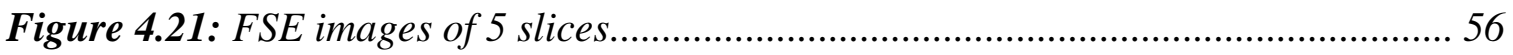

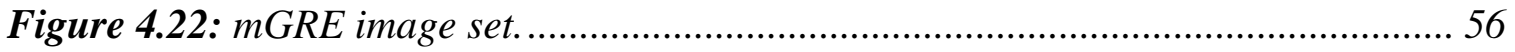

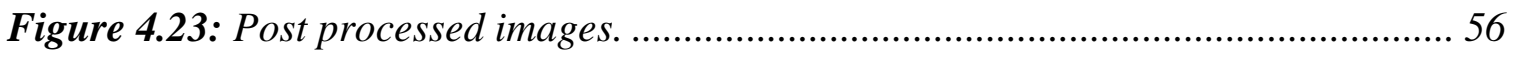

Figure 4.24: ROI selection for $T_{2}{ }^{*}$ calculations. ................................................... 57

Figure 4.25: Plot indicating correlation of $\mathrm{pO}_{2}$ with $\mathrm{T}_{2} *$ at the region of probe tip...... 59

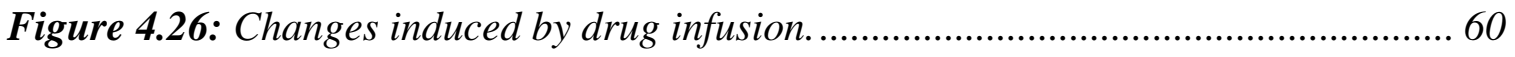

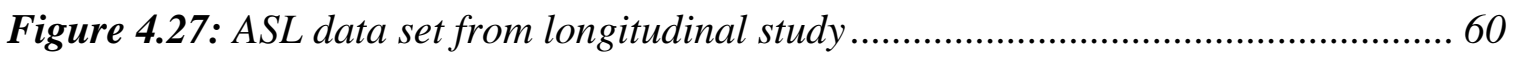

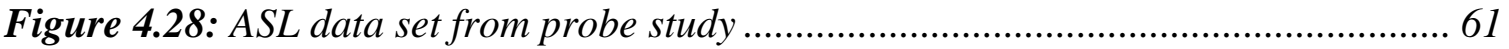

Figure A.1: Protons in magnetic field aligned along the external magnetic field $B_{0} \ldots . . .70$

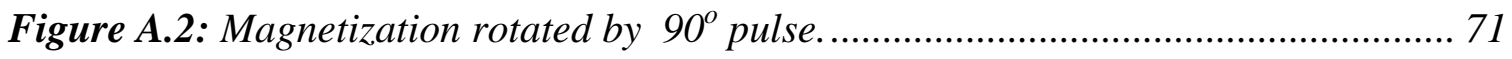

Figure A.3: Dephasing of transverse magnetization during free induction decay......... 71

Figure A.4: Longitudinal magnetization recovery and tissue contrast........................... 72

Figure A.5: Transverse magnetization decay and contrast. ...................................... 73

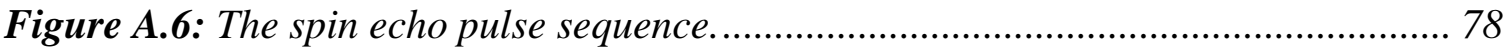

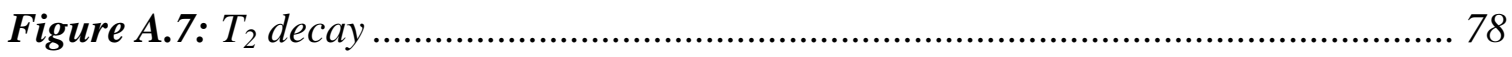

Figure A.8: A generic spin-echo pulse sequence diagram.......................................... 79

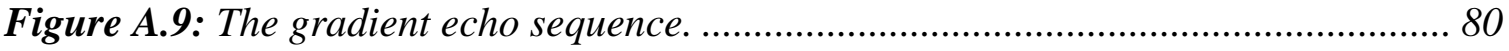




\section{LIST OF TABLES}

Table 1.1: Oxygen consumption of the kidney compared to other organs in humans........ 4

Table 1.2: Prevalence of type 1 and type II diabetes in USA........................................ 6

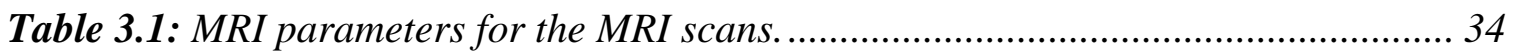

Table 3.2: Animal parameters recorded at the onset of the experiments........................ 35

Table 3.3: Imaging time points and body weights..................................................... 38

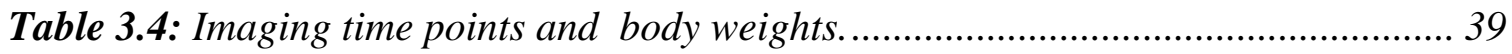

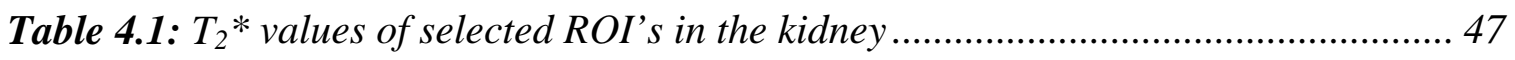

Table 4.2: Table indicating the change of MAP at different time periods. ...................... 53

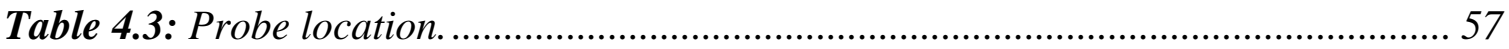

Table 4.4: Oxygenation readings of both $\mathrm{MRI}$ and $\mathrm{pO}_{2}$ probe..................................... 58 


\section{CHAPTER 1: INTRODUCTION}

\subsection{Introduction to MRI}

Magnetic Resonance (MR) Imaging (MRI) is a state-of-the-art imaging technology, which allows cross sectional viewing of the body with unprecedented variation in tissue contrast. The past 15 years have witnessed an explosion of information regarding the role of magnetic resonance imaging in assessing pathologic conditions. MRI is considered much safer than x-ray or computed tomography (CT) as it uses only non-ionizing electromagnetic fields and has rapidly evolved into an accepted modality for medical imaging of disease processes throughout the body.

MRI is based on the principles of nuclear magnetic resonance (NMR), a spectroscopic technique used by scientists to obtain microscopic chemical and physical information about molecules. The technique is called magnetic resonance imaging rather than nuclear magnetic resonance imaging (NMRI) because of the negative connotations that became associated with the word nuclear in the late 1970's. MRI started out as a tomographic imaging technique that produces an image of a slice through the human body. Magnetic resonance images provide a three dimensional digital representation of tissue characteristics that can be obtained in any spatial orientation. Since its inception, MRI has expanded beyond a tomographic imaging technique; it is also capable of true volume imaging.

MRI has become a preferred method for diagnosing problems in many parts of the body. MRI is very helpful, providing noninvasive imaging of soft tissue, such as organs, muscle, cartilage, ligaments, and tendons. While x-rays or CT are best for viewing bones, MRI can identify and show the difference between healthy and unhealthy tissue. With MRI, it is often possible to diagnose disease at very early stage, and before it is visible by any other means. Because most disease are best treated when found early, MRI scans are becoming increasingly useful. 
This study concerns the development of an MRI technique for detecting abnormalities in kidney functioning and validating the obtained results with real-time measurements using more standard invasive laboratory equipment. The MRI technique developed here are for measuring oxygenation and perfusion in the kidney.

\subsection{Motivation and Objective of this thesis}

Noninvasive in vivo MRI measures of regional kidney microvasculature and structure provide new ways to detect and track renal hemodynamic changes. Two well established functional MRI methods, blood oxygenation level dependent (BOLD) MRI [1] and arterial spin-labeling (ASL) [2] perfusion MRI are particularly applicable to the study of renal hemodynamics. Imaging methods are especially useful because they provide not just one measurement, but rather images of relative oxygenation and perfusion throughout the kidney. While there has been significant progress in the development of these two functional MRI methods for renal applications, neither method has yet been validated against standard invasive laboratory measurements. That validation of MRI by invasive optical probe measurements is the primary objective of this work. In addition, if these techniques are to be useful in the clinic, they must have documented measures of reliability. That is the other goal that was accomplished by this work.

Studies have proven BOLD and ASL can individually provide useful information about the kidney, as they measure an index of oxygenation and quantitative perfusion respectively. This study is the first validation of these two non-invasive techniques against standard invasive methods. The objective of this study is to develop and test in vivo MRI techniques to provide a novel, reliable, non-invasive way to detect two types of changes:

1. Renal oxygenation: $\mathrm{BOLD}$ will be validated with $\mathrm{pO}_{2}$ (partial pressure of $\mathrm{O}_{2}$ ) as an index of renal oxygenation, and

2. Renal perfusion: ASL with laser-Doppler flow (LDF) for renal blood perfusion. 
As this study deals with kidney oxygenation and perfusion measurement, a brief description of kidney anatomy and function is provided. The following sections cover kidney physiology and then discuss one of the potential applications of this study.

\subsection{Functional Anatomy of the Kidney}

The principal function of the mammalian kidney is to maintain homeostasis or equilibrium between the human body internal fluid volume and electrolyte status and also that of the environment's influences, diet and intake. It functions to maintain the intra and extracellular fluid volume despite variation in daily fluid and electrolyte intake. In man, the kidneys consist of roughly 2 million nephrons and weigh approximately 250 grams [3]. The kidneys' extraordinary excretory and regulatory objectives are achieved through the processes of glomerular ultrafiltration, tubular reabsorption and tubular secretion.

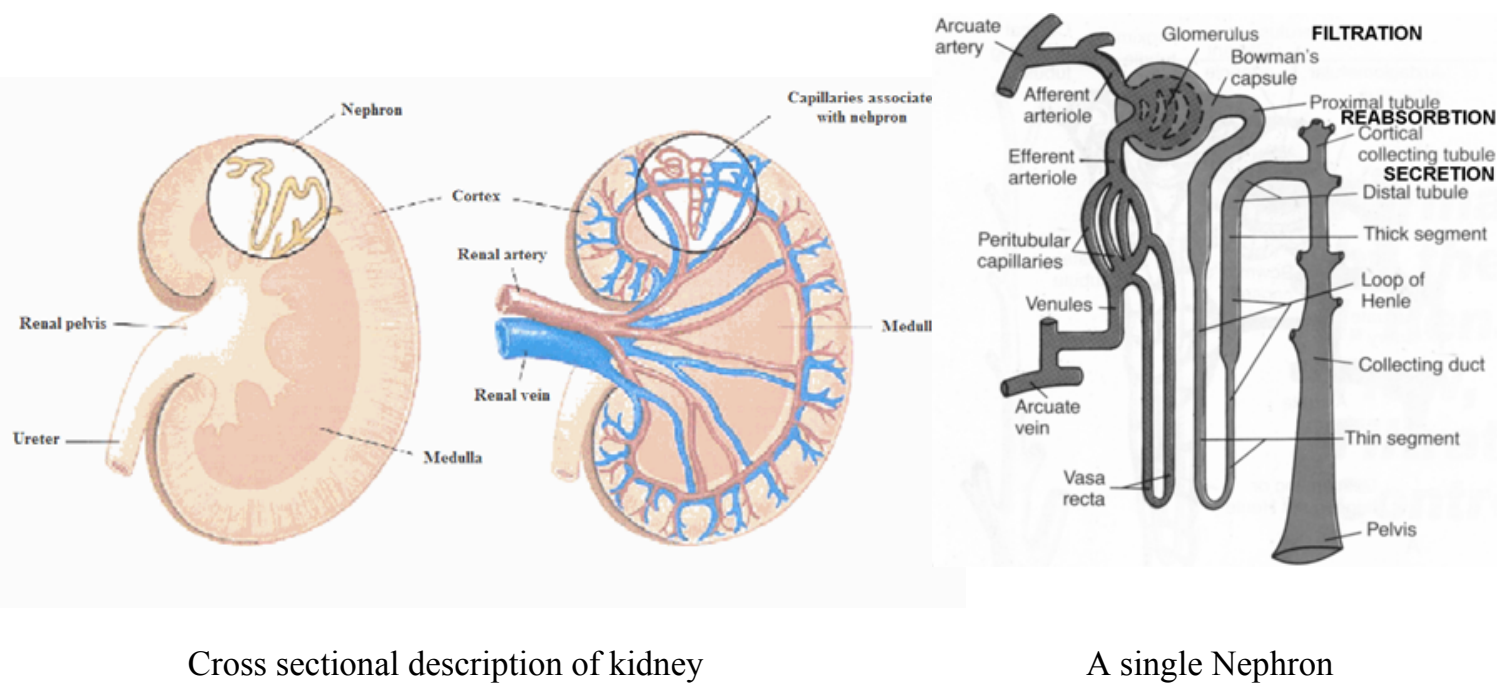

Figure 1.1: Kidney anatomy and functioning. [6]

The initial step in the glomerular filtration is the formation of a plasma ultrafiltrate (plasma without cells or proteins) at Bowman's space through the action of hydrostatic pressure in the glomerular capillaries. Then the ultrafiltrate flows along the tubules and is modified by re-absorption of important solutes (sodium salts, glucose, amino acids) and most water from the lumen of the tubules back into the peritubular capillary blood [3]. Finally, the luminal fluid is also modified by secretion of solutes from 
the peritubular capillaries (or from the tubule cells) into the lumen. Filtration is major function of the kidney, which is greatly dependent on oxygenation and blood flow.

\subsubsection{Renal Blood Flow and Oxygenation}

These excretory and regulatory processes depend on the blood supply to the kidney. It receives the highest blood flow per gram of organ weight in the body at 1 liter/min, i.e. $20 \%$ of the cardiac output [4]. Thus, a very substantial portion of the total cardiac output flows through the kidneys.

Table 1.1: Oxygen consumption of the kidney compared to other organs in humans. [6]

\begin{tabular}{|c|c|c|c|c|}
\hline $\begin{array}{c}\text { Region or } \\
\text { Organ }\end{array}$ & $\begin{array}{c}\mathrm{O}_{2} \text { Delivery } \\
\mathrm{ml} / \mathrm{min} / 100 \mathrm{~g}\end{array}$ & $\begin{array}{l}\text { Blood Flow } \\
\text { Rate } \\
\mathrm{ml} / \mathrm{min} / 100 \mathrm{~g} \\
\end{array}$ & $\begin{array}{c}\mathrm{O}_{2} \\
\text { Consumption } \\
\mathrm{ml} / \mathrm{min} / 100 \mathrm{~g} \\
\end{array}$ & $\begin{array}{c}\mathrm{O}_{2} \\
\begin{array}{c}\text { Consumption/O } \\
\text { Delivery (\%) }\end{array} \\
\end{array}$ \\
\hline Hepatoportal & 11.6 & 58 & 2.2 & 18 \\
\hline Kidney & 84.0 & 420 & 6.8 & 8 \\
\hline $\begin{array}{l}\text { Renal outer } \\
\text { medulla }\end{array}$ & 7.6 & 190 & 6.9 & 79 \\
\hline Brain & 10.8 & 54 & 3.7 & 34 \\
\hline Skin & 2.6 & 13 & 0.38 & 15 \\
\hline Skeletal muscle & 0.5 & 2.7 & 0.18 & 34 \\
\hline Heart & 16.8 & 87 & 11.0 & 65 \\
\hline
\end{tabular}

Oxygen consumption by the kidneys is quite high, amounting to about $8 \%$ of the total oxygen consumption of the body. Oxygen delivery to any organ is directly dependent on hemoglobin saturation $\left(\mathrm{HbO}_{2} \%\right)$ and cardiac output (blood flow). As in other tissues, sufficient blood flow provides adequate oxygenation and nutrition. Therefore, the relatively high blood flow to the kidneys feeds its high metabolic demands caused by the glomerular filtration process. [3]

Blood flow to the kidneys is dependent on a number of systemic factors. A lowered blood volume (dehydration, hemorrhage) or cardiac output (congestive heart failure, myocardial infarct), causes diminished blood flow. In less obvious ways, hypoalbuminemia (cirrhosis, nephrotic syndrome, and starvation) affects the intravascular 
volume so that the effective blood (volume) flow is diminished despite normal hydration. Also, hypotension (abnormal low pressure of blood) from severe vasodilatation or hypertension from constricted or occluded vessels can diminish blood flow to the kidneys.

A gradient of renal oxygenation exists in the kidneys with the lowest partial pressure of oxygen $\left(\mathrm{pO}_{2}\right)$ in the renal medulla [5]. Due to the low $\mathrm{pO}_{2}$ in the renal medulla, the cells in this region are at constant risk of hypoxic (deficiency of $\mathrm{O}_{2}$ ) injury. Although numerous studies have shown that renal medulary cells may adapt well to low $\mathrm{pO}_{2}$, the precise mechanism mediating this adaptive response in healthy individuals' remains poorly understood [6] and is even less understood in various renal diseases.

Furthermore, the factors responsible for progressive renal failure are not fully understood. Risk factors appear to be the severity of the primary disease and complications such as uncontrolled hypertension, urinary tract infection, obstruction, or the intrarenal deposition of calcium phosphate. However, even when these factors are controlled by surgical corrections and prevention of infection, many disorders can continue to progress to end stage renal disease (ESRD). Thus it has been suggested that after a critical point, a reduction in the number of functioning nephrons eventually results in the failure of all the nephrons [4].

\subsection{Potential Application of this study}

These observations form the basis for current clinical studies that are attempting to reverse such hemodynamic changes and thus slow the progression to ESRD [7]. A serious threat to renal health is diabetic nephropathy, which develops over course of diabetes and is often detected only at a later stage when loss of kidney function has already occurred [8] .

\subsubsection{Diabetic Nephropathy (DN)}

Diabetic nephropathy is the kidney disease that can occur as a result of diabetes. It is a leading cause of kidney failure in Europe and the USA. After many years of diabetes, 
the delicate filtering system in the kidney becomes destroyed by the effects of high blood glucose, initially becoming leaky to large blood proteins such as albumin, which are then lost in urine. This is more likely to occur if the blood sugar has been poorly controlled. The studies in this thesis are targeted toward applications in this patient population.

\subsubsection{Prevalent Statistics}

In the United States, approximately 17 million people, or $6.2 \%$ of the population, are estimated to have diabetes, with a growing incidence. Roughly one third of this population is estimated to be undiagnosed with type II diabetes. The overall risk of developing diabetic nephropathy varies between about $10 \%$ of type II diabetics (noninsulin-dependent diabetes of late onset) to about $30 \%$ of type I diabetics (insulindependent diabetes of early onset).

Table 1.2: Prevalence of type 1 and type II diabetes in USA. [9]

\begin{tabular}{|c|c|c|}
\hline \hline & $\begin{array}{c}\text { Type I } \\
\text { Diabetes }\end{array}$ & $\begin{array}{c}\text { Type II } \\
\text { Diabetes }\end{array}$ \\
\hline \hline $\begin{array}{c}\text { Prevalence of } \\
\text { disease* }\end{array}$ & $0.85-1.7$ million & $\begin{array}{c}15.3-16.2 \text { million (estimated } \\
5.9 \text { million undiagnosed?) }\end{array}$ \\
\hline \hline $\begin{array}{c}\text { Prevalence of microalbuminuria at } \\
15 \text { years }\end{array}$ & $21 \%$ & $28 \%$ \\
\hline $\begin{array}{c}\text { Progression to end-stage renal } \\
\text { disease 10 years after onset of } \\
\text { macro-albuminaria }\end{array}$ & $50 \%$ & $10 \%$ \\
\hline
\end{tabular}

High blood pressure almost always develops or worsens in diabetic nephropathy, and can be the first abnormality to develop. DN, which is a microvascular complication of diabetes, is also a sign of worsening blood vessel disease throughout the body. Larger blood vessels (arteries \& veins) are almost always affected leading to heart attacks, strokes, and circulatory disease occurring more often and at a younger age than usual [9]. 


\subsubsection{Different stages of DN}

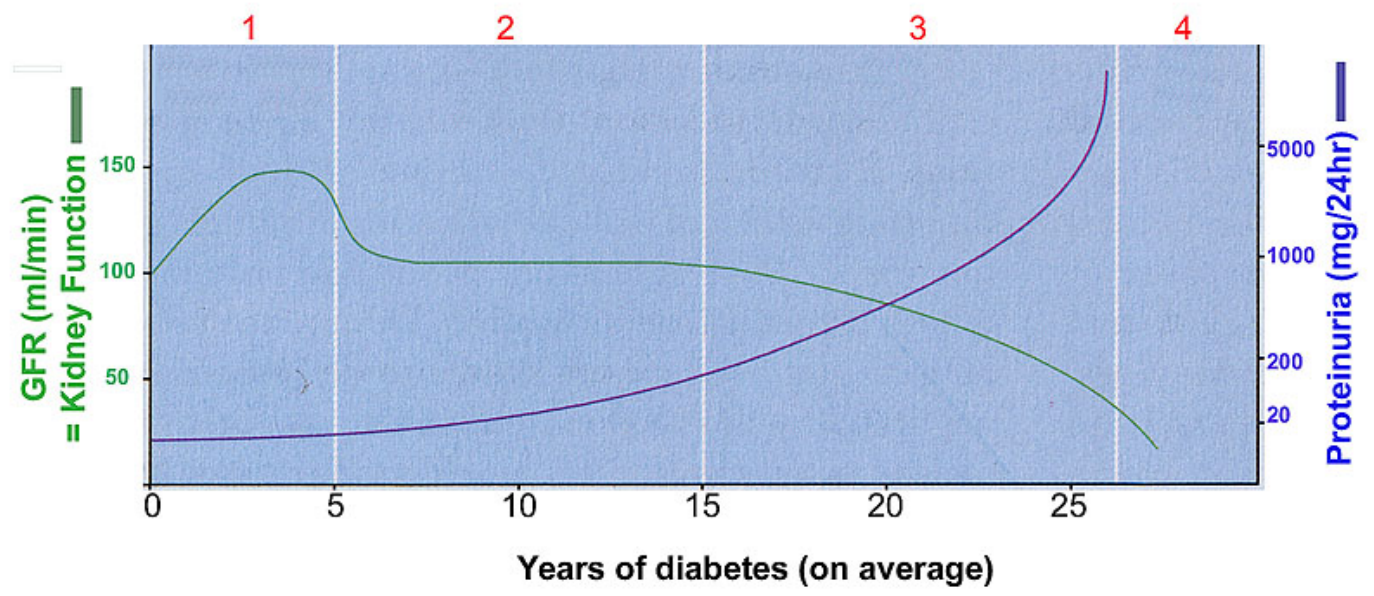

Figure 1.2: Plot indicating kidney failure over time in DN. [10]

1. Begins with a tiny amount of the protein, albumin, appearing in the urine microalbuminuria. In $\sim 30 \%$ of type II diabetes, this protein can reverse [11].

2. Over 10-15 years' proteinuria increases and nephritic syndrome may develop.

3. The development of proteinuria is indicative of the kidneys' worsening inability to remove poisons from the blood.

4. This is called "end-stage renal disease" (ESRD). Dialysis is required or kidney transplantation and, unless so treated, the poisons can build up to fatal levels [10].

\subsubsection{Need for Early Detection and Diagnosis}

Current testing strategies are not able to predict DN before the onset of microalbuminuria and in some cases this may not be predictive. Like DN, various other kidney diseases like hypertension, hypotension, etc. require longitudinal monitoring of kidney physiology [12]. Present monitoring and testing strategies are not highly reliable and so there is an immediate need for early detectors [13]. 


\section{CHAPTER 2: METHODS AND DETAILS}

\subsection{Blood Oxygenation and Level Dependent (BOLD) MRI}

BOLD MRI is a noninvasive method capable of providing regional measurements of relative percent saturation of hemoglobin and thus indirectly tissue oxygenation. The basic principle of BOLD is that magnetic field perturbations caused by paramagnetic molecules lead to a loss of phase coherence and therefore signal attenuation in (gradient echo) $\mathrm{T}_{2}{ }^{*}$-weighted sequences [14]. Since deoxyhemoglobin (as opposed to oxyhemoglobin) is paramagnetic, a change in the hemoglobin saturation reflective of tissue oxygenation would modify tissue $\mathrm{T}_{2}{ }^{*}$. Indeed, this method has been proven capable of detecting changes in renal oxygenation during administration of drugs, indicating its potential to detect pathophysiologic changes in renal oxygenation reflected in hemoglobin saturation changes and blood flow changes. [15]

\subsubsection{BOLD-MRI Details}

The BOLD effect is the basis of most of the functional MRI studies done today to map patterns of activation in human brains [13]. In brain, when an area of cortical tissue experiences an increased metabolic demand in response to increased activity, the brain responds by increasing blood flow and oxygenation to the active region [16]. The BOLD signal reflects the MR signal change when the magnetic susceptibility of blood is altered by this rise in the concentration of oxyhemoglobin and the associated increase in blood flow, reducing field gradients around the vessels leading to an increase in the MR signal. A quantitative model of this process has been developed for understanding the basic mechanisms of this effect and for calibrating the signal to the local oxygen consumption rate $\left(\mathrm{CMRO}_{2}\right)[17]$.

\subsubsection{BOLD Mechanism}

The connection between BOLD changes and tissue oxygenation is through the relationship between oxygen and hemoglobin in the blood. Deoxygenated hemoglobin has unshielded iron atoms that directly modify the local magnetic fields causing a decrease in the MRI signal. 


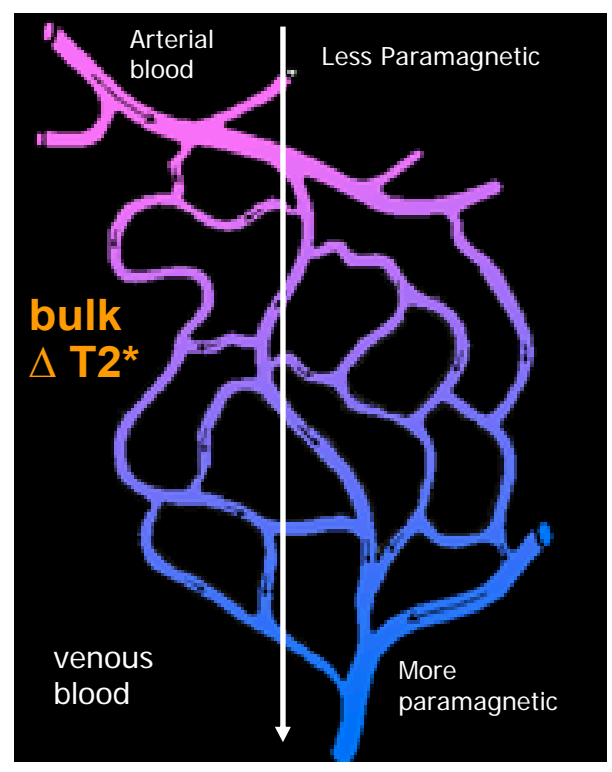

Paramagnetic effect leading to $\mathrm{T}_{2} *$ increase

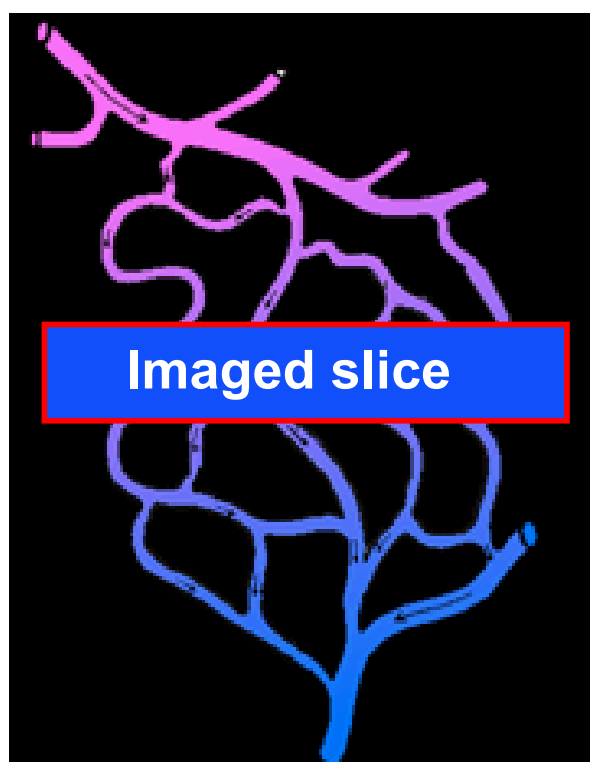

Blood inflow leading to $\mathrm{T}_{1} *$ changes

Figure 2.1: BOLD mechanism.

Blood flowing through the arteries has high percentage of free oxygen and oxygen bound to hemoglobin. As the blood passes in the tissue, the oxygen is released by hemoglobin and absorbed by the tissues and thus the percentage of oxygen in the vessels goes down increasing the level of deoxygenated hemoglobin in the blood [18]. As hemoglobin is paramagnetic, a susceptibility change is created which is revealed during sequential imaging. The exact effect is of a decrease in the effective spin-spin relaxation time, $T_{2}{ }^{*}$. (As $T_{2}{ }^{*}$ is inversely related to its effective relaxation rate $\left(R_{2}{ }^{*}\right), T_{2} *=1 / R_{2}{ }^{*}$, the $\mathrm{T}_{2} *$ decrease can be described as an $\mathrm{R}_{2} *$ increase). Thus, as the percentage of oxygenated hemoglobin $\left(\% \mathrm{HbO}_{2}\right)$ decreases during circulation, the MRI relaxation time $\mathrm{T}_{2} *$ decreases and the rate $\mathrm{R}_{2} *$ increases as the blood circulates.

\subsubsection{BOLD-MRI Details}

While it has been demonstrated [19] that these $\mathrm{T}_{2} *$ changes are correlated with changes in oxygenation induced by pharmacological or physiological challenges, they have not been directly related to oxygenation values in the kidney. As mentioned above, studies of the mechanism for the BOLD-MRI signal increases observed in kidney [20]and 
in the brain studies $[21,22]$ have determined that the BOLD effect has two components, changes in $\% \mathrm{HbO}_{2}$ and changes in blood flow. The MRI signal can be expressed as:

$$
\begin{gathered}
S\left(R_{1}^{*}, R_{2}^{*}\right)=M_{o} \frac{\sin (\alpha) e^{-T E \cdot R 2^{*}}\left(1-e^{-T R \cdot R_{1} *}\right)}{\left(1-\cos (\alpha) e^{-T R \cdot R_{1}^{*}}\right)} ; \\
R_{1}^{*}=R_{1}-\frac{f}{\lambda} ; \\
R_{2}^{*}=C \cdot \frac{\left(1-\% H b O_{2}\right)}{B V} .
\end{gathered}
$$

The variables represent the BOLD-MRI parameters: $\boldsymbol{M}_{\boldsymbol{o}}=$ the equilibrium magnetization, $\mathrm{TE}=$ is the echo time, TR is the repetition time, and $\alpha$ is the flip angle. The variable $f$ is blood flow and $\boldsymbol{\lambda}=0.9$ is the tissue partition coefficient, $\mathbf{C}$ is a constant dependent on magnetic field, vessel size and vessel orientation. The relaxation constants are $\mathrm{R}_{1} *$ and $\mathrm{R}_{2} *$ (equal to $1 / \mathrm{T}_{1} *$ and $1 / \mathrm{T}_{2} *$ ). Thus changes in $\% \mathrm{HbO}_{2}$ and blood volume (BV) changes cause $T_{2} *$ changes and flow changes cause $T_{1} *$ changes $[16,21,22]$.

While it has been shown that the $\mathrm{T}_{2}$ * changes in the medulla of the kidney are associated with challenges that correspond to vascular and $\mathrm{pO}_{2}$ changes, [21,22] this has been inferred, but not verified. Separation of the two components of the BOLD signal can be accomplished by a linear fit to the natural log of the multi-echo signal (obtained from mGRE pulse sequence) measurements to extract the slope $\left(\mathrm{T}_{2}{ }^{*}\right)$ and intercept (related to $\left.\mathrm{T}_{1}^{*}\right)[21]$.

This study aims to correlate the relative changes in BOLD signal to a $\mathrm{pO}_{2}$ measurement by an invasive method. Although flow changes observed in the intercept, this measure is relatively insensitive, so a different technique, called Arterial Spin Labeling (ASL) has been implemented for perfusion quantification and is explained in detail in the following section. 


\subsection{Arterial Spin Labeling}

Arterial spin labeling (ASL) is the term used for a class of MR techniques in which arterial water is used as an endogenous tracer for quantitative perfusion measurement. The arterial water is magnetically labeled such that the magnetizations of blood and tissue are different. Arterial water flowing into the imaging slice, exchanges with tissue water and changes the magnetization of the latter. The quantification of the perfusion uses the principle described above, that the relaxation time, $\mathrm{T}_{1}{ }^{*}$, is altered by flow.

ASL measurements generally conform to the following pattern:
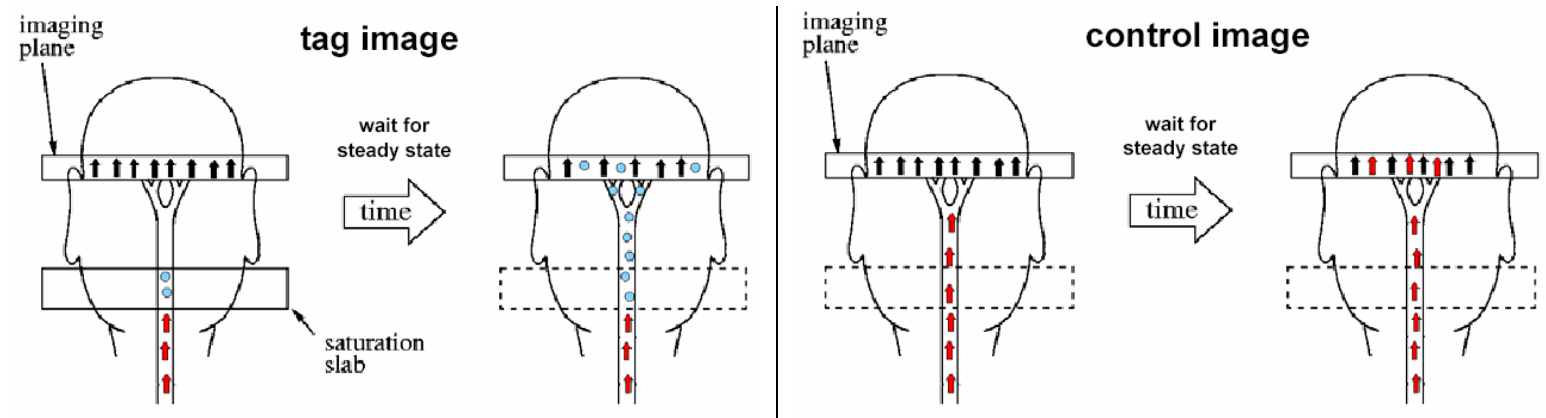

$\begin{array}{ll}1 & \text { normal blood } \\ 0 & \text { saturated blood }\end{array}$

$\uparrow$ tisue

1 normal blood
saturated blood

Control Image acquisition

Tagged Image acquisition

Figure 2.1: ASL imaging. [23]

- The magnetization of arterial water is inverted or saturated upstream to the imaging slice. This constitutes the labeling or tagging of the blood.

- A delay is allowed for the tagged blood to reach the imaging slice and exchange with tissue water. The arterial magnetization relaxes due to $T_{1}$ relaxation during this period.

- The magnetization is measured in the imaging slice(s). This magnetization is a mixture of the tissue magnetization and the change in the magnetization due to inflow of tagged blood.

- A control image is acquired in the same manner as above, except that the arterial water isn't labeled. 
- The difference between the two images is dependent only on the amount of magnetization of the tagged blood that entered the imaging slice and exchanged with tissue water. Since the magnetization of the tagged blood is less in the tagged image than in the control image, the tagged image is subtracted from the control image.

- The difference between the two images is only on the order of $1 \%$ of the static tissue magnetization. The perfusion-weighted differences images therefore suffer from inherently low SNR. Repetition of the measurements and averaging is therefore required. Imaging is usually done with alternating tag and control images.

A variety of different ASL sequences following the above principles have been developed since 1992. ASL sequences generally fall into one of the two categories, depending on how the tag is applied, continuous ASL and pulsed ASL [22], both wellestablished techniques in brain. The explanation of the working principles for these two methods follows; brain imaging used as an example.

\subsubsection{Continuous Arterial Spin Labeling (CASL)}

In CASL arterial spins are inverted using continues RF irradiation as they pass through an inversion plane as shown in figure (2.2). The resonant frequency of the blood would then be spatially dependent such that arterial magnetization would experience a frequency sweep as it passed through the inversion plane.

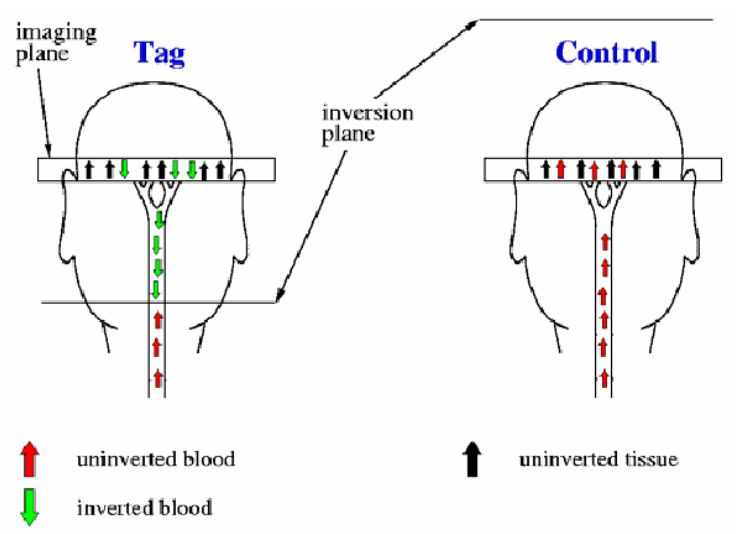

Figure 2.2: Continuous tagging and simultaneous acquisition. [23] 
These 'labeled' spins flow into the capillary beds of the brain and exchange with tissue water, alternating the overall tissue magnetization. Since the arterial spins are inverted continuously, a steady state is reached in which the magnetization of brain tissue within an imaging plane is directly related to the cerebral blood flow $(f)$ and is given as follows:

$$
f=\frac{\lambda}{T_{\text {lapp }}} \frac{M_{\text {cont }}-M_{\text {inv }}}{2 M_{\text {cont }}}
$$

Here, $\lambda$ is the blood-brain partition coefficient, $T_{\text {lapp }}$ is the apparent longitudinal relaxation time constant, $\mathrm{M}_{\text {cont }}$ is the longitudinal magnetization per gram of brain tissue (i.e. signal intensity) for a control image obtained with out spin inversion, and $\mathrm{M}_{\mathrm{inv}}$ is the magnetization for an image obtained without inversion. This type of CASL measurements is mostly applicable with single-slice imaging where the imaged slice is parallel to the inversion plane. Certain issues like the effects of intravascular signal and long transit delay times make this technique impotent when compared to PASL. This study uses PASL for perfusion imaging as explained in detail in the following section [24].

\subsubsection{Pulsed Arterial Spin Labeling (PASL)}

In the PASL scheme, the spin inversion is achieved using short inversion pulses, typically 10-15 ms long, which inverts spins in a specific region, known as the inversion slab [17]. The transit delays can be measured using multiple inversion times although this is a very time consuming process with limited SNR. Alternately, the sensitivity towards transit delays can be overcome by applying a saturation pulse in both the tag and control experiments. [22] Thus, the spatial distribution of delay times is measured and corrected for perfusion calculations.

\subsubsection{Perfusion Quantification}

The general PASL perfusion quantification is described in this section. Adiabatic fast passage RF pulses are used to label spins in the inflowing blood, which inverts the 
spins at a plane proximal to the region where perfusion is measured. The tissue perfusion rate $(f)$ is given with the following equation:

$$
f=\frac{\lambda}{2 \alpha T_{1 a p p}} \bullet \frac{\left(M_{b}^{s s 1}-M_{b}^{s s 2}\right)}{M_{b}^{s s 1}},
$$

Here, $M_{b}^{s s 1}$ is the steady state tissue water magnetization per gram of tissue that is reached when macromolecular spins are saturated, without arterial spin labeling, and $M_{b}^{s s 2}$ is the steady state tissue water magnetization per gram of tissue reached with arterial spin labeling and macromolecular saturation. The symbol $\lambda$ is used for the bloodtissue partition coefficient of water in milliliters per gram. The degree of labeling of arterial blood $\alpha$ is defined as by the following:

$$
\alpha=\frac{\left(M_{a}^{o}-M_{a}\right)}{2 M_{a}^{o}},
$$

Here, $M_{a}^{o}$ is the equilibrium arterial blood magnetization per milliliter of blood, and $\mathrm{M}_{\mathrm{a}}$ is the magnetization per milliliter of labeled arterial blood. For perfect inversion, $M_{\mathrm{a}}=-M_{a}^{o}$ and $\alpha=1$, and for saturation of arterial spins, $M_{\mathrm{a}=0}$ and $\alpha=0.5$. $\mathrm{T}_{\text {lapp }}$ is the measured $T_{1}$ in the absence of saturation of macromolecules and is defined by the following:

$$
\frac{1}{T_{1_{a p p}}}=\frac{1}{T_{1 b}}+\frac{f}{\lambda},
$$

Here, $T_{1 b}$ is the intrinsic $T_{1}$ of the tissue water. With this definition, $T_{1 a p p}$ is sensitive to changes in tissue perfusion as well as the $T_{1}$ of tissue water. The effects of cross relaxation between water and macromolecules have been neglected in the definition of $\mathrm{T}_{1 \text { app }}$ because at high field, its contribution to the measured $\mathrm{T}_{1}$ has been shown to be small [17].

PASL and CASL are conceptually very similar, but each has its own advantages and limitations. PASL is a more diverse set of techniques than CASL, with several variations in different applications. Some of the acronyms one may encounter for PASL techniques include EPISTAR, PICORE, FAIR, UNFAIR and QUIPS. These various 
methods differ in their technical detail based on their applications. In this study, the FAIR technique has been implemented in this thesis work for quantizing perfusion using ASL.

\subsubsection{Flow-sensitive Alternating Inversion Recovery (FAIR)}

The FAIR method is based on the acquisition of two images: the first image is preceded by a nonselective $180^{\circ}$ - pulse (global inversion) then the image is acquired after an inversion time $T_{i n v}$, during which the spin system returns to equilibrium with the spinlattice relaxation time $T_{1}$. The second image is preceded by a slice selective $180^{\circ}$ inversion applied to the imaging slice, while all other parameters remain unchanged. In the case of slice-selective inversion, non-inverted spins enter the imaging slice during $T_{i n v}$ in the presence of blood flow. in the case of slice-selective inversion, while there are no non-inverted spins that would enter the imaging slice after global inversion. In the subsequent image acquisition, these non-inverted spins result in an increased signal after slice-selective inversion compared to global inversion. Subtraction of the image preceded by non-selective inversion from the image taken after selective inversion gives a signal intensity mapping reflecting local difference in spin inflow caused by tissue perfusion [24].

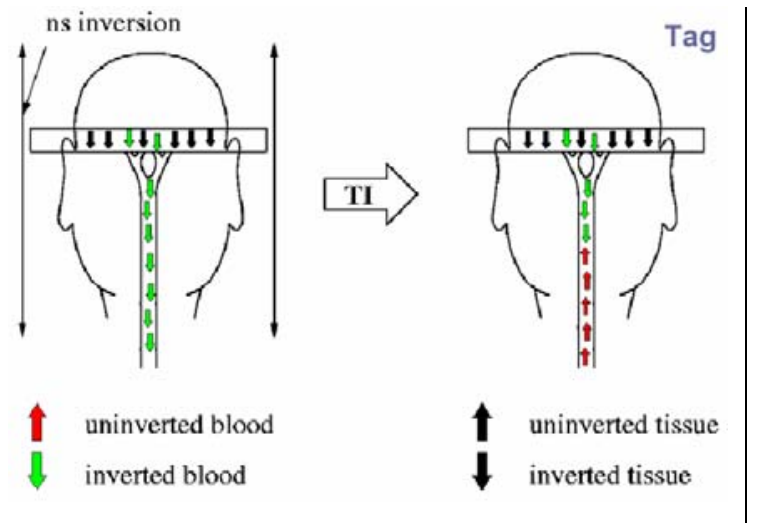

Tagging sequence
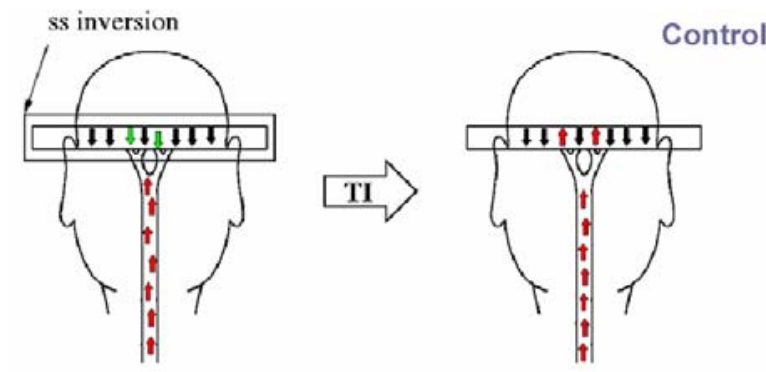

uninverted blood
$\Downarrow \quad$ inverted blood

Control sequence

Figure 2.3: ASL-FAIR imaging. [23]

The time-evolution of the magnetization after nonselective $\left(\mathrm{M}_{\mathrm{ns}}(\mathrm{t})\right)$ and sliceselective $\left(M_{s s}(t)\right)$ inversion is given by: 


$$
\begin{aligned}
& M_{n s}(t)=M_{o}\left(1-2 \exp \left(-t / T_{1}\right)\right) \\
& M_{s s}(t)=M_{o}\left(1-2 \exp \left(-t / T_{1}^{s s}\right)\right)
\end{aligned}
$$

$T_{1}$ is the spin lattice relaxation time and $T_{1 s s}$ is the apparent relaxation time after slice selective inversion of the spins. When the capillary-tissue exchange of blood water is instantaneous, the $\mathrm{T}_{1 \mathrm{ss}}$ is given by:

$$
\frac{1}{T_{1}^{s s}}=\frac{1}{T_{1}}+\frac{f}{\lambda}
$$

Where, $\lambda$ the tissue/blood water distribution coefficient (assumed to be $0.8 \mathrm{~mL} / \mathrm{g}$ ) $[\mathrm{X}]$, and $f$ is the perfusion rate in $\mathrm{ml} / \mathrm{g} / \mathrm{s}$. The difference image intensity at the inversion time $\Delta \mathrm{M}\left(\mathrm{T}_{\mathrm{inv}}\right)$, is then given by:

$$
\begin{gathered}
\Delta M\left(T_{i n v}\right)=M_{s s}-M_{n s} \\
\Delta M\left(T_{i n v}\right)=-2 M_{o} \exp \left(\frac{-T_{i n v}}{T_{1}}\right)\left(1-\exp \left(\frac{-T_{i n v} f}{\lambda}\right)\right)
\end{gathered}
$$

Since $f / \mathrm{L}$ is small (f $\sim 0.05 \mathrm{ml} /(\mathrm{g} / \mathrm{min})$ ), the last term can be expanded, and the equation now reads:

$$
\Delta M\left(T_{i n v}\right)=-2 T_{i n v} M_{o} \frac{f}{\lambda} \exp \left(\frac{-T_{i n v}}{T_{1}}\right)
$$

The perfusion ' $f$ ' is then given by

$$
f=\frac{-\Delta M \lambda}{2 T_{i n v} M_{o}} \exp \left(\frac{T_{i n v}}{T_{1}}\right)
$$

Perfusion maps can thus be calculated pixel-by-pixel from the knowledge of magnetizations $\mathrm{M}_{\mathrm{ns}}$ and $\mathrm{M}_{\mathrm{ss}}$, the undisturbed intensity $\mathrm{M}_{\mathrm{o}}$, and the spin lattice relaxation time $\mathrm{T}_{1}$. 


\subsection{Literature Survey}

\subsubsection{BOLD MRI Technique}

The group led by Dr. P. Prasad has done pioneering research in the field of BOLD-MRI [19]. In 1994, they demonstrated the feasibility of this noninvasive method using a sequential measure of response to a variety of physiological / pharmacological stimuli in health and disease in humans. On a set of young healthy human subjects, acetozolamide and furosemide, drugs known to change kidney $\mathrm{pO}_{2}$, were infused to observe the BOLD signal changes in the kidney by MRI. The spin-spin relaxation rate $\mathrm{R}_{2} *\left(1 / \mathrm{T}_{2}{ }^{*}\right)$ was measured, which is a measure of deoxyhemoglobin in blood and in turn relates to tissue $\mathrm{pO}_{2}$. The results concluded that BOLD-MRI can be used to monitor changes in intrarenal oxygenation in humans in a non-invasive fashion.

In 1999, the use of BOLD in rats was implemented using a multiple gradient recalled echo (mGRE) sequence. This is the same sequence which has been used in this thesis work. It was observed that the loop diuretic furosemide increased $\mathrm{T}_{2}{ }^{*}$, inferring that it improved the oxygenation in the renal medulla. These results were complemented by earlier invasive studies that measured oxygenation using oxygen microelectrodes [25].

The reproducibility of intra-renal $\mathrm{T}_{2} *$ and $\Delta \mathrm{T}_{2} *$ measurements after drug infusion, has also been studied [26]. Results indicated that $\mathrm{T}_{2}{ }^{*}$ measurements were reproducible over 270 days within $12 \%$ and furosemide produced a significant and reliable change $(\sim 30 \%)$.

Ries et. al. carried out an interesting study applying BOLD in rats with diabetic nephropathy [13]. They investigated the possibility of using diffusion weighted imaging (DWI-MRI) along with BOLD to detect pathological and physiological changes induced by chronic renal hyperfiltration. They implemented multiple gradient echo (mGRE) imaging for BOLD in 17 rats including furosemide infusion. They concluded that BOLD imaging may be able to detect tissue at risk from ischemia by revealing information about the balance between tubular workload and delivery of oxygen in rats. 
The first attempt to correlate BOLD contrast with $\mathrm{pO}_{2}$ in tumors was performed on a 4.7T magnet. Tumor $\mathrm{pO}_{2}$ was measured using fiber-optic microprobes similar to those used in this thesis. They concluded, that the major advantages of the BOLD technique included non-invasiveness, high spatial resolution and real time detection of $\mathrm{pO}_{2}$ fluctuations. They further concluded that information offered by the BOLD imaging technique in tumors is qualitative in nature and can be combined with other techniques capable of providing an absolute measure of $\mathrm{pO}_{2}$.

\subsubsection{Arterial Spin Labeling (ASL) MRI Technique}

In 1992, a technique was developed for proton MRI of perfusion imaging, using water as a freely diffusible tracer [27]. The first application was for the measurement of regional cerebral blood flow $(\mathrm{rCBF})$ in rats. The method involved labeling the inflowing water proton spins in the arterial blood by inverting them continuously (CASL) at the neck region and observed the effects of inversion on the intensity of brain MRI. They clearly demonstrated the techniques sensitivity to changes in $\mathrm{rCBF}$ and the ability to detect region abnormality in perfusion.

In 1994, the same group demonstrated this technique in rat kidneys [17]. Quantitative renal perfusion images were obtained in rats, by measurement of the reduction in kidney MR image signal intensity after steady state magnetic labeling of arterial blood in the suprarenal aorta. Labeling was achieved with adiabatic fast passage inversion of arterial water. They obtained cortical renal blood flow values, which correlated with previous studies done with other techniques, there by indicating the methods potential applicability in humans.

In 1997, ASL technique was implemented in transplanted rat kidney for perfusion quantification [28]. Cortical perfusion was quantified from a slice through the center of each kidney at $4.7 \mathrm{~T}$ with a fast gradient echo MRI sequence following the ASL. Over a series of post kidney transplanted measurements, the renal cortical perfusion rate determined by MRI significantly correlated with the histological rejection. They concluded that these measurements of renal cortical perfusion could provide a non- 
invasive diagnostic method for monitoring the status of renal transplants without requiring the administration of contrast agents.

In 2000, renal perfusion quantification was demonstrated using ASL with the FAIR sequence [24]. Quantitative perfusion imaging of human kidneys was performed using arterial spin labeling MRI with a fast spin-echo. Intra-individual reproducibility errors were smaller than the natural inter-individual variability of renal perfusion. Perfusion in the cortex was approximately 3-4 times higher than that of the medulla. They concluded that the ability to quantify perfusion, the lack of ionizing radiation and lack of contrast medium made this technique attractive for diagnosing renal pathologies that are associated with reductions in tissue perfusion.

\subsubsection{Validation of MRI with Invasive Techniques}

In 2004, a study was conducted on the effect of 4 different types of anesthesia on mice at 4.7T [29]. The BOLD-MR signal intensity in tumors was compared with flow measurements by laser Doppler flowmetry and oxygen measurements by luminescencebased probes. Simultaneous BOLD imaging was done along with tumor blood flow and $\mathrm{pO}_{2}$ measured by the same OxyLite/OxyFlo probe system used for this thesis. The results from BOLD-MRI were not in direct correlation with the standard measurements of oxygenation and blood perfusion but had a non-linear relationship. This was predictable as the hemoglobin saturation relationship to tissue oxygenation is best described by a sigmoidal curve. Also, the need was emphasized for careful monitoring of the effects of anesthesia when trying to identify new therapeutic approaches that are aimed at modulating tumor hemodynamics. It is notable that tumors are highly heterogeneous and their results are not obviously extendable to other tissue types.

\subsection{Approach to the problem}

This study focuses on testing the utility of BOLD- and ASL-MRI techniques for the first time in rat kidney. As validation is the main objective of this study, standard invasive laboratory method was required [30]. After a careful consideration of available 
methods to invasively measure renal oxygenation and blood flow, the optical probe system was selected for this study. Following are the prime advantages of the optical probes when compared to the other existing methods:

1. The probes are MRI compatible and can be custom manufactured for probe length.

2. Both oxygenation and blood flow probes are integrated in a single probe, thereby decreasing tissue damage and multiple insertions. But more importantly, we get a measurement of blood flow and $\mathrm{pO}_{2}$ at the same place.

\subsubsection{Optical Probe Oxygenation and Blood Flow Measurement Methods}

Oxygen availability is critical to metabolism and is an important parameter in many bio-reactions. A number of methods have been developed for making measurements of oxygen in vivo such as polographic electrodes, fluorescence quenching, EPR technique, and biopsy-based technique and NIR spectroscopy [26]. The fluorescence quenching system was used for this study as an invasive measurement.

\subsubsection{Fluorescence Quenching Oximetry}

Fluorescence quenching (FQ) sensors are based on state-of-the-art optical technology for continuous quantitative monitoring of regional $\mathrm{pO}_{2}$ in tissue and fluids. This novel technology offers several key advantages over other existing devices, making these oxygen sensors much easier to use, better suited to physiological oxygen measurements and sensitive enough to detect of tissue hypoxia; $0<\mathrm{pO}_{2}<100 \mathrm{mmHg}$ [31].

The FQ $\mathrm{pO}_{2}$ probes are based on fiber optics and provide a continuous measure of oxygen partial pressure coupled with fast response times for real-time monitoring of temporal oxygen changes. Short pulses of blue LED light are transmitted along the fiber optics to excite a platinum-based fluorophore situated at the probe tip. The fluorophore is permanently immobilized and enclosed within a silicone matrix. The resulting emission of fluorescent light, quenched by the presence of oxygen molecules, travels back up the fiber and is detected by the instrument. The lifetime of fluorescence is inversely 
proportional to the concentration of dissolved oxygen, and is interpreted, after calibration, to provide an absolute value for $\mathrm{pO}_{2}$ in $\mathrm{mmHg}$ or $\mathrm{kPa}$. Fluorescence lifetime is longest at low $\mathrm{pO}_{2}$, making probes most sensitive in the physiological range 0-80 $\mathrm{mmHg}$. Also, as the measurement is based on fluorescence lifetime rather than fluorescent intensity it is much less prone to artifacts (e.g. due to variation in the intensity of the light source, ambient lighting, or photo-bleaching, etc.). Compensation for the effects of temperature is required for temperature changes greater than $7^{\circ} \mathrm{C}$ since fluorescent lifetimes are affected by changes in temperature. Temperature is measured by a fully integrated thermocouple, allowing simultaneous monitoring of tissue $\mathrm{pO}_{2}$ and temperature as well as automatic temperature correction [31].

The unique features of these oxygen sensors make them well suited to measuring tissue oxygenation during MRI when compared to conventional polarographic techniques. The FQ-based sensors do not show significant oxygen consumption, thus the sensors can not only be used to gain spatial $\mathrm{pO}_{2}$ information, but can be left in position for monitoring the long-term, temporal evolution in tissue $\mathrm{pO}_{2}$. These sensors are compatible for use in MRI and spectroscopic applications. Furthermore multiple sensors can be used to provide simultaneous temporal oxygen data from several tissue sites, which is ideal for monitoring the effects of treatment regimes and pharmacological agents.

\subsubsection{Laser Doppler Flowmetry (LDF)}

Laser Doppler flowmetry is an established technique for the real-time measurement of microvascular red blood cell (or erythrocyte) perfusion in tissue. Perfusion is sometimes referred to as microvascular blood flow or red blood cell flux. Laser Doppler signals from the tissue are recorded in BPU (Blood Perfusion Units) which is a relative scale defined using a carefully controlled motility standard comprising a suspension of latex spheres undergoing Brownian motion [31].

Laser Doppler Flowmetry (LDF) works by illuminating the tissue under observation with low power laser light from a probe composed of two optical fiber light 
guides. Laser light from one fiber is scattered within the tissue and some is scattered back to the probe. Another optical fiber collects the backscattered light from the tissue and returns it to the monitor. Most of the light is scattered by tissue that is not moving but a small percentage of the returned light is scattered by moving red blood cells. The light returned to the monitor undergoes signal processing whereby the emitted and returned signals are compared to extract the Doppler shift related to moving red blood cells. Thus, the laser-Doppler blood perfusion monitors output a signal that is proportional to the red blood cell perfusion (or flux). This represents the transport of blood cells through microvasculature and is defined as:

\section{Microvascular Perfusion Number of blood cells \\ (Red Blood Cell Flux) moving in the tissue $\quad \mathbf{x} \quad$ Mean velocity of these cells sampling volume}

As LDF defines a perfusion parameter from information contained in the optical spectrum of light remitted from the tissue, the actual measurement sampling volume or depth can only be determined by identifying precisely which blood vessels and erythrocytes have interacted with the remitted light. This in turn, is principally dependant on two parameters; namely the optical scattering and optical absorption coefficients of the tissue under observation. Since both of these coefficients are entirely dependent on the site of observation and perfusion of the microvasculature at the time of measurement, it is impossible to determine the actual sampling volume/depth at any tissue site.

The LDF technique offers substantial advantages over other methods in the measurement of microvascular blood perfusion during MRI. Studies have shown that it is both highly sensitive and responsive to local blood perfusion and is also versatile and easy to use for continuous monitoring. Measurements obtained by LDF are intrinsically of a relative nature. Although such measurements are proportional to perfusion, the factor of proportionality will be different for different tissues. [23] 


\section{CHAPTER 3: EXPERIMENTAL DESIGN AND SPECIFICATIONS}

\subsection{Technical Specifications}

\subsubsection{Goals of the Study}

As described in section 1.2, this thesis work was undertaken with the following goals:

1. To measure the reliability of $\mathrm{T}_{2}{ }^{*}$ and ASL in rat kidneys by performing a longitudinal study at $3 \mathrm{~T}$ field strength.

2. To correlate measurements from non-invasive MRI methods to that of invasive optical methods in the kidney.

Six male Sprague-Dawley rats were studied in the WVU-HSC 3.0T MRI scanner. Baseline MRI measurements were acquired for the longitudinal study in order to test the stability of $\mathrm{T}_{2}{ }^{*}$ maps over time as well as to get control values. The probe study was

conducted to correlate the invasive with the non-invasive measurements. By the simultaneous acquisition of data from BOLD- \& ASL-MRI, and the optical probes inserted into the kidney.

Goal 1: Baseline values for all normal rats did not involve any sort of surgery. An external kidney holder was used to isolate and immobilize the kidney. The longitudinal baseline measurements gave the normal renal oxygenation index, $\mathrm{T}_{2}{ }^{*}$, and perfusion for each individual animal. Data was acquired for:

\section{1) Regional renal oxygenation index, $T_{2} *$ using BOLD MRI imaging 2) Regional renal perfusion using arterial spin labeling (ASL) MRI.}

Goal 2: The probe study involved a non-recovery surgery during which the probe was inserted into the kidney and catheters were inserted for drug infusion. Imaging along with the optical probe was done for three time periods as control, angiotensin II infusion and furosemide infusion. Data was acquired for: 


\section{1) Regional renal oxygenation and microperfusion using BOLD MRI imaging and Fluorescence Quenching (FQ) $\mathrm{pO}_{2}$. \\ 2) Regional renal perfusion using arterial spin labeling (ASL) MRI and Laser Doppler Flow (LDF) measurement.}

A combined optical probe for FQ tissue oxygenation and laser Doppler blood flow measurement system (Oxford Optronix) was used to acquire perfusion and oxygenation data in vivo simultaneously during the imaging to verify MRI measures [1, 29]. The specifications of MR pulse sequences and the optical probe apparatus are presented in the following sections.

\subsubsection{MR Imaging Pulse Sequences}

Kidney localization is a prime task at the onset of imaging. Initially a 3 plane localizer is helpful to locate the kidney in the image. One of the benefits of the pulse sequence technique is that all three planes are automatically acquired.

\subsubsection{Fast Spin Echo (FSE) Imaging}

FSE is an MRI pulse sequence that rapidly acquires refocused echoes. Unlike the standard spin echo method, the FSE technique changes the phase-encoding gradient for each of these echoes. As a result of changing the phase encoding gradient between echoes, multiple lines of k-space can acquired within a given TR. The number of echoes acquired in a given TR is called the echo train length (ETL), which ranges from 4 to 16. The echo spacing between successive echoes is a function of the bandwidth. Higher bandwidths permit the quicker acquisitions but at the expense of lower SNR [32]. The FSE sequence may also be used to acquire thinner slices with an increased signal to noise ratio $(\mathrm{SNR})$.

As multiple phase-encoded lines are acquired during each TR interval, these techniques significantly reduce imaging time. For instance, an FSE sequence with an ETL of eight can be performed in one-eight the time of a conventional spin echo sequence with the same TR. 


\subsubsection{Spoiled Gradient Echo (SPGR)}

Spoiling refers to the purposeful disruption of transverse magnetization coherences, which may persist between cycles in a gradient echo sequence when the TR is very short. (i.e. TR $<5$ to $100 \mathrm{~ms}$ ). This ensures that there is no residual transverse steady-state magnetization before it can be acted upon by the next RF pulse. Although small $\mathrm{T}_{2} *$ contributions are introduced by the gradient reversal, mostly $\mathrm{T}_{1}$ weighted contrast is obtained acquired with small TR, short TE and with low to moderate flip angle acquisition [32]. Usually, gradient spoiling is performed by turning on the slice-select gradient for an additional time after data acquisition, with variable amplitude at the end of each cycle immediately before the next RF pulse.

In this thesis work, fat suppressed 3-dimensional (3D) volumetric interpolated sequences 3D-SPGR was used to image thin slices $(1.6 \mathrm{~mm})$ of the left kidney in the axial plane. Twelve oblique slices were selected across the left kidney to get a good coverage of the kidney in the axial view. Minimum slice thickness was opted for both of these sequences to obtain the greatest possible detail. The 3D-SPGR was averaged for eight times to increase the signal to noise ratio (SNR).

\subsubsection{BOLD-MRI: Renal Oxygenation}

Tissue oxygenation states and tissue metabolic activity were evaluated using BOLD contrast imaging. BOLD imaging was performed through the kidneys using the mGRE pulse sequence. Images at 8 echo times, 4.2, 8.08, 11.9, 15.8, 19.7, 23.6, 27.5, $31.3,35.2 \mathrm{~ms}$, were acquired to calculate $\mathrm{T}_{2}{ }^{*}$. Raw data from four acquisitions was averaged together before image reconstruction to maximize SNR and minimize motion artifacts in the images. Images were collected of axial slices through the left kidney.

In this study, a fast spin echo sequence was used for acquiring anatomical image of the kidney. The field of view was maintained at $12 \mathrm{~cm}$, same as that of the mGRE images. The echo train length (ETL), [the number of echoes collected in a single TR] used was eight. The main advantage with FSE is the acquisition at images with long TR 
and $\mathrm{TE}$ in a reasonable time. Increasing ETL reduces SNR, acquisition time and greatly increases signal homogeneity.

\subsubsection{Multiple Gradient Echo (mGRE)}

The mGRE pulse sequence involves acquisition of multiple echoes after each excitation pulse [9]. Unlike the conventional GRE, the mGRE provides images at different echo times. This technique permits calculation of $\mathrm{T}_{2} *$ from the $\mathrm{T}_{2} *$ weighted images [10]. The utility of this sequence in this study would be to monitor the druginduced $\mathrm{T}_{2} *$ changes in a region of interest. Figure 3.1 indicates the pulse timing diagram of the mGRE sequence for four echoes. After the $90^{\circ}$ excitation pulse, a gradient is applied at an interval of $4.95 \mathrm{~ms}$. At the successive timings the data acquisition (DAQ) window is opened to collect the respective echo.

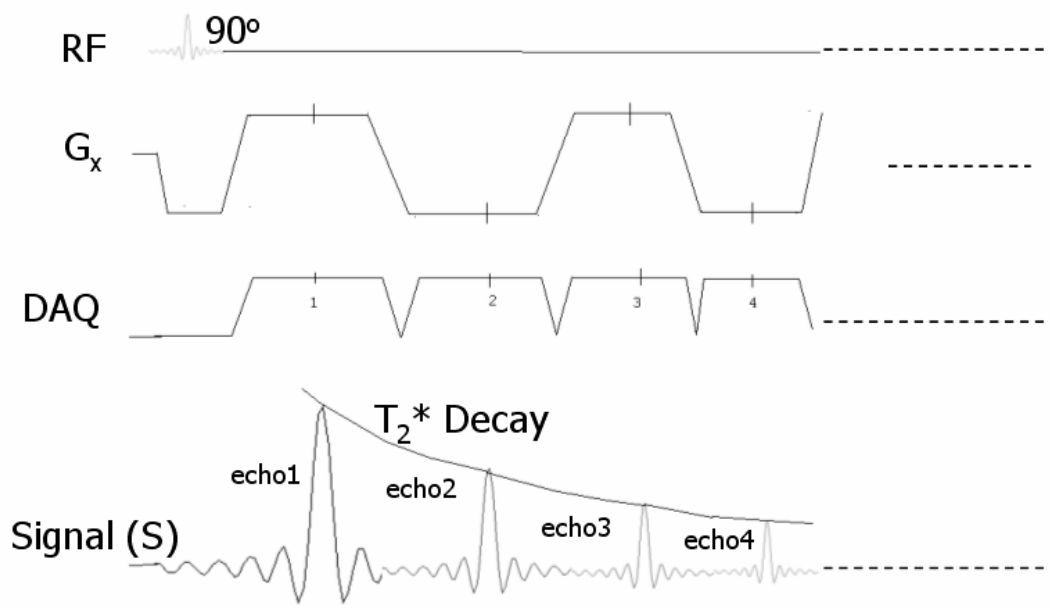

Figure 3.1: mGRE pulse timing diagram.

The amplitude of the signal echo reduces from $1^{\text {st }}$ echo to the $8^{\text {th }}$ echo. The slope of logarithm of the signal intensity vs. the echo time represents the $\mathrm{T}_{2} *$ decay.

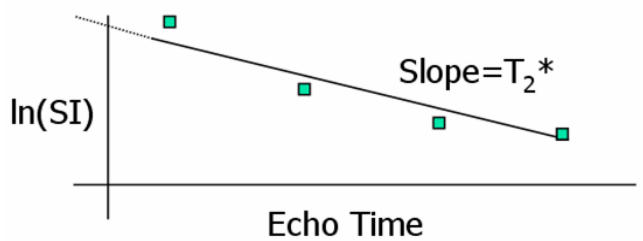

Figure 3.2: Plot between $\ln (S I)$ and echo time indicating the $T_{2} *$ decay. 


\subsubsection{Perfusion MRI using Arterial Spin Labeling}

The technique used to measure perfusion is based on tagging a population of water protons in flowing blood within a segmented volume, and subsequently following these water protons on subsequent temporally spaced images to track the flowing spins. The FAIR sequence is used for obtaining the tagged and untagged images. As the direction of the blood flow is not known, FAIR (Flow sensitive Alternating Inversion Recovery) was the best choice available for implementing ASL.

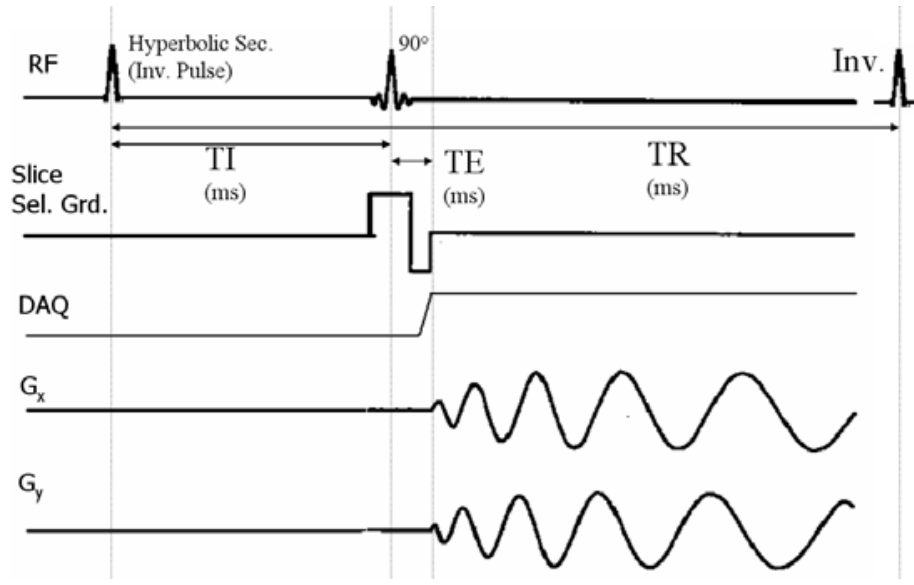

FAIR-ASL pulse timing diagram

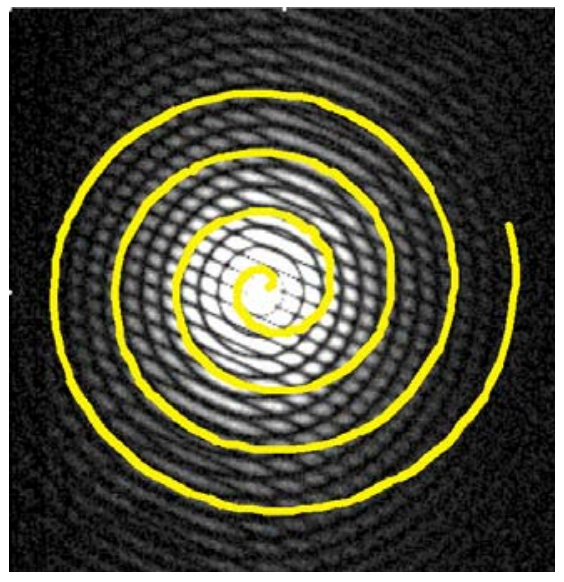

Spiral acquisition strategy

Figure 3.3: FAIR-ASL pulse sequence.

As shown in the timing diagram of ASL in figure 3.3, the hyperbolic secant pulse is applied to apply an inversion over the entire imaging slab. After an inversion time period (TI), a $90^{\circ}$ pulse is applied along with the slice selective gradients. After an echo time period of TE, the DAQ window is opened to collect the signal. Here, the $G_{x}$ and $G_{y}$, are applied in opposite phase to obtain a spiral trajectory.

\subsubsection{Dual Probe OxyLab/OxyFlo System}

The combination of the oxygenation and flow sensors into a single probe provides simultaneous measurements of tissue blood flow and $\mathrm{pO}_{2}$. The combined $\mathrm{pO}_{2}$ and LDF probes are supplied in a 'bare-fiber' format for invasive yet minimally traumatic tissue monitoring applications. A suitable gauge needle or catheter is generally used to deliver the probe to the tissue. Bare-fiber style probes (without the temperature function) are 
fully MRI-compatible and are available at extended fiber lengths for this type of application. The tip diameter is $450 \mu \mathrm{m}$ and the probe cables are manufactured from white flexible PVC tubing with an external diameter of $3.0 \mathrm{~mm}$ and standard length of 2 $\mathrm{m}$. An additional cable adapter of $6 \mathrm{~m}$ is used to connect the probe located in the MRI to the monitors that are located remotely in the control room.

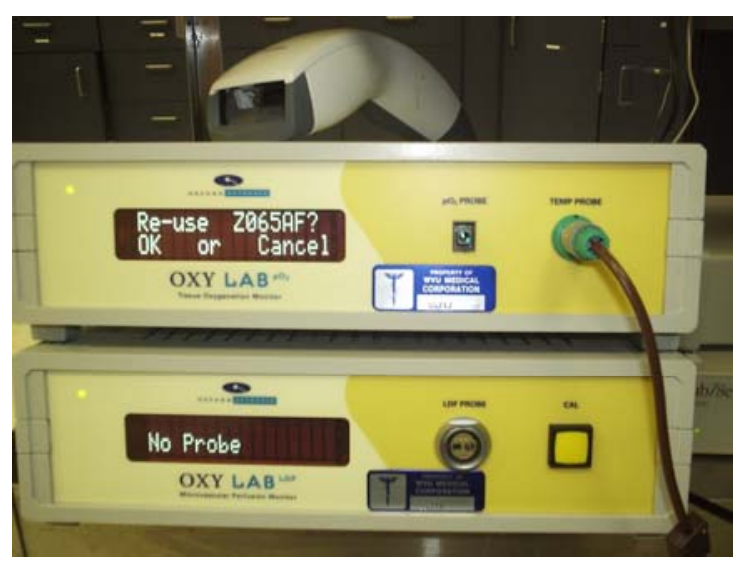

Photograph of the monitors

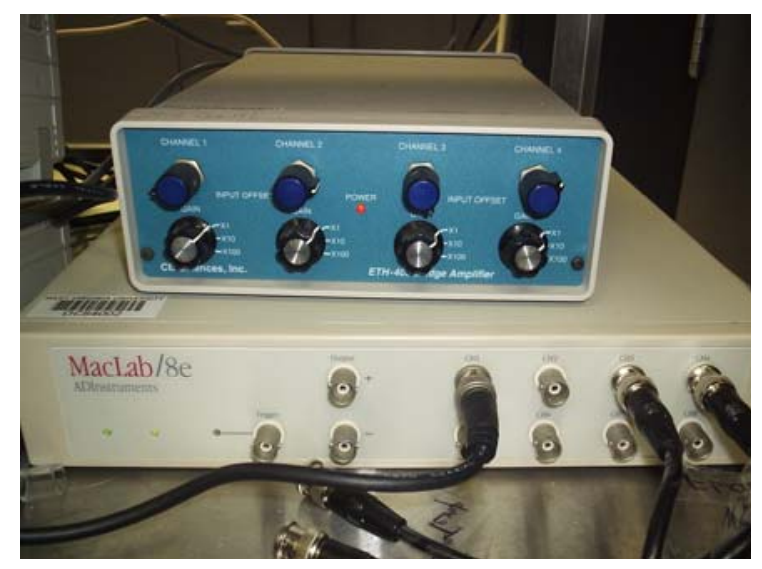

Photograph of the MacLab (DAQ)

Figure 3.5 Set up for real-time invasive oxygenation and perfusion measurements.

ADInstruments MacLab data acquisition device was used to acquire the data on to a Macintosh. The MacLab software had real time display of the connected $\mathrm{pO}_{2}$ and LDF measurements.

\subsubsection{OxyLab Fluorescence Quenching Oximetry}

The OxyLab $\mathrm{pO}_{2}$ (Oxford Optronix) is an instrument capable of continuous, quantitative measurement of dissolved partial pressure of oxygen $\left(\mathrm{pO}_{2}\right)$ and temperature in tissue, physiological fluids, cell cultures and in vitro samples.

As described in Chapter 2, OxyLab $\mathrm{pO}_{2}$ is an optical device based on the principle that the presence of oxygen in tissue or fluids can terminate (quench) light emitted by a luminescent compound (dye). The quenching of the light is proportional to the oxygen tension in the vicinity of the dye. The system consists of portable monitor and detachable single-use, disposable probe. Within the monitor, there is a blue light source, sensitive photodetector and signal processing electronics. Each probe is comprised of a single 
optical fiber that conducts light from the monitor to the sensing tip. The sensing tip at the distal end of the fiber contains the luminescent dye held in a polymer matrix. When the dye is illuminated/excited with blue light from the monitor, it luminescences. The presence of oxygen in its path quenches the luminescence. The returned quenched light is sent through the signal processor, which determines the luminescence decay time. The corresponding $\mathrm{pO}_{2}$ is displayed on the monitor in units of $\mathrm{mmHg}$ or kilo Pascal's $(\mathrm{kPa})$. The monitor can display a range of $0-100 \mathrm{mmHg}$ with a response time of less than $10 \mathrm{sec}$.

Since measured $\mathrm{pO}_{2}$ is dependent on temperature, the OxyLab $\mathrm{pO}_{2}$ incorporates a calibrated temperature measurement system. The fluorescent lifetimes are affected by temperature changes of more than $7^{\circ} \mathrm{C}$. Compensation for the effects of temperature will not be required as temperature changes no larger than $3^{\circ} \mathrm{C}$ were observed during the entire experiment. The microprobe fiber optic cables that were used for these experiments were 9 meters long to reach into the magnet bore from the control room.

When the probe was placed in homogeneous tissue, the $\mathrm{pO}_{2}$ reading was relatively insensitive to motion artifact arising from movements of the tissue (e.g. due to breathing or ventilation). As the different area of the rat kidney is less the $2 \mathrm{~mm}$ thick between the regions of the medulla, it was important to ensure that the probe and the tissue move together. However, continuous movement of the probe relative to the tissue may result in signal variability due to an inconsistent sampling region and may also cause tissue damage or local tissue trauma. Care was taken to ensure that undue probe pressure was not applied to the tissue, since local occlusion of the microvasculature may impact on the $\mathrm{pO}_{2}$ reading. This was done by suspending the probe from a custom built plastic holder attached to the plexiglas table. Direct illumination of external light source was avoided as ambient lighting at the probe measurement site might disrupt the $\mathrm{pO}_{2}$ reading.

\subsubsection{OxyLab Laser Doppler Flowmetry}

The OxyLab LDF is a laser Doppler microvascular perfusion monitor that is capable of monitoring red blood cell (erythrocyte) perfusion in the microcirculation of a tissue. As described in Chapter 2, this monitor uses a technique commonly referred to as 
laser Doppler flowmetry (LDF), an established and reliable method for the measurement of blood perfusion in microvasculature research.

The OxyLab LDF perfusion monitor works by illuminating tissue with low power laser light, using a probe containing optical fiber light guides. The system incorporates semiconductor laser diode devices operating in continues mode and emitting invisible laser radiation at a nominal operating wavelength of $830 \mathrm{~nm}$. The maximum output power at the probe tip is less than $0.5 \mathrm{~mW}$. The laser light emitted from the optical fiber is highly divergent and is scattered within the tissue and some is scattered back to the probe. Another optical fiber collects the backscattered light from the tissue and returns it to the monitor. Most of the light is scattered by tissue that is not moving but a small percentage of the returned light is scattered by moving red blood cells. The light returned to the monitor undergoes signal processing to extract the signal related to the moving red blood cells. Microvascular blood perfusion is indicated on the front panel display in relative units called Blood Perfusion Units (BPU). The monitor can display a range of 09999 BPU's with a response time of less than 1 sec.

The presence of motion artifact noise in the blood perfusion signal is often due to relative movements of the tissue (e.g. induced by animal breathing) with respect to the probe and/or probe cable movements. These artifacts were minimized by allowing the probe to come in contact with the tissue such that the probe and tissue 'move together' and by ensuring no movement of the cables.

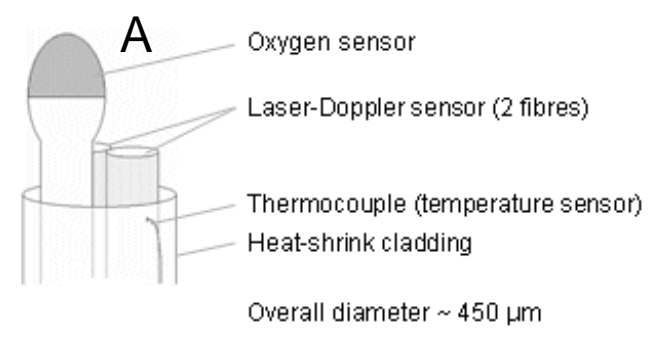

The schematic of the probe

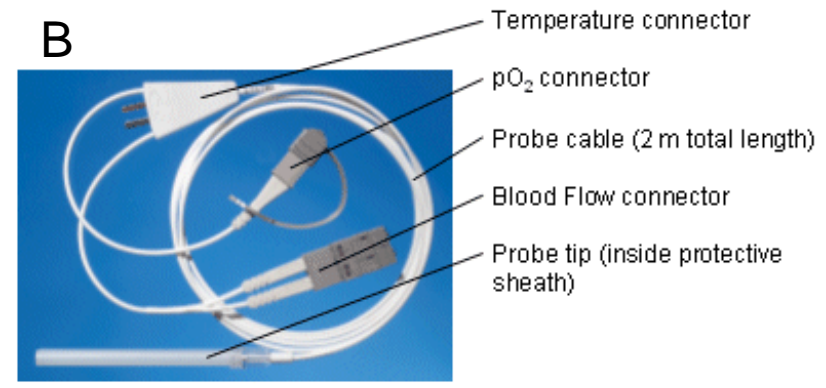

A Picture of the probe

Figure 3.4. Combined $L D F$ and $\mathrm{pO}_{2}$ probe. 


\subsubsection{MR Compatible Motion Detector}

Small rodents, like rats, positioned in the magnet bore are no longer visible from the scanner control room and so while scanning the investigator must have a remote "eye" to observe the animal. Without such a device, the experiment may be rendered invalid by unrealized physical changes in the animal. A device capable of detecting motion may be used to monitor the animal respiratory waveform and to alert the investigator to changes in the animal position. The animal breathing rate and depth of inspiration/expiration are indicative of certain physiologic responses to procedures, including body temperature changes, heart rate changes and depth of anesthesia. For MRI experiments, a respiration monitor serves an additional purpose, because techniques that are used to suppress breathing-related motion artifacts in the images require a respiratory signal [14].

An MR-compatible respiratory motion detection system for small rodents must meet certain requirements. To be MR compatible, the monitor must be insensitive to the MR system's electromagnetic fields and must not degrade image quality. To be useful for small animals like rats, it must be sensitive enough to measure small respiratory motion.

Design Specifications: The setup consists of a lab-built custom-made printed circuit board (PCB) with 4 sets of Infrared emitter-detector pairs along with inverted amplifier circuitry. An aluminum assembly was specially designed and fabricated to connect the IR pairs to the fiber optic cables (25' long). An IR emitter and detector were used in the circuitry. The emitter used is a high output infrared LED which emits IR light in the range of $1.2 \sim 1.6$ Volts. The two leads of the emitter were connected to two potentiometers of each $10 \mathrm{~K}$ and $1 \mathrm{~K}$ in order to control the amount to current into the circuit. The photo detector used detects the IR light from the emitter and gives a corresponding output. When a motion occurs, the change in the motion is detected by the detector and is given to external amplification circuit, which is then amplified and displayed. 
Data Acquisition: The motion detector PCB was connected to MacLab Hardware (ADInstruments, USA) and data acquisition was done on PowerLAB (ADInstruments, USA). A pair of cables was placed on the animal chest. Due to the IR radiation absorption by the animal skin, a reflective hologram sticker was placed on the animal, approximately $5 \mathrm{~mm}$ from the cables. The software displayed displacement waveform from the setup, which was the motion sensed from the animal. The displayed data can be saved as a file for further analysis.

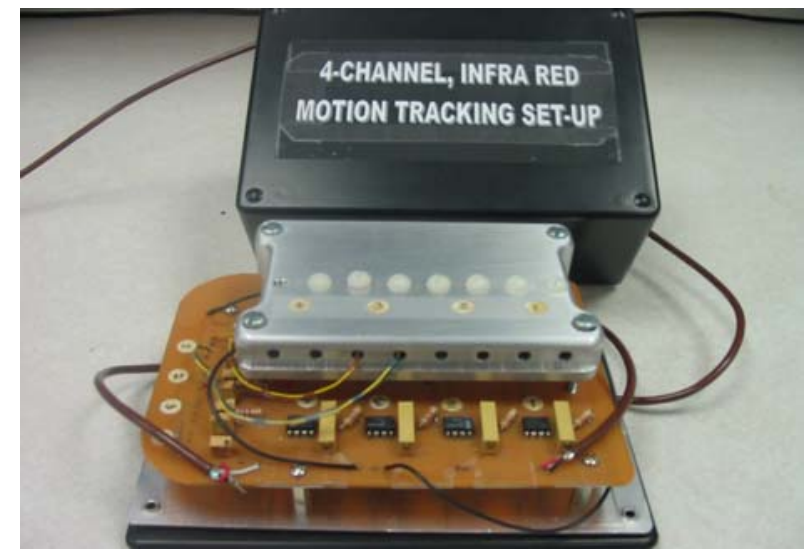

Motion tracking circuitry

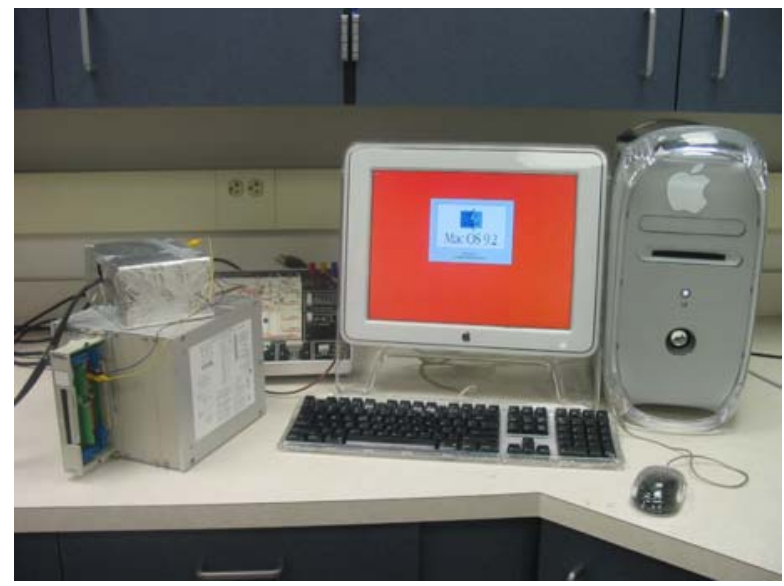

The entire set-up

Figure 3.6: The motion detector 


\subsection{MRI \& Probe Experimental Design \& Procedures}

The longitudinal and probe studies were carried out independently. For the longitudinal MRI experiments the animals were scanned in series. The MRI-probe studies were a non-survival experiments done on one animal at a time as these studies involved surgery and pre- and post- drug infusion periods.

\subsubsection{Image Acquisition}

This study was conducted according to a protocol accepted by then Animal Care and Use Committee, Health Sciences Center, WVU. All of the imaging was done on GE 3.0T MRI system (General Electric, SIGMA, Milwaukee, Wisconsin). A 7" diameter, extremity coil was used for RF transmission and reception. Each set of imaging sequences took $\sim 40$ min. scan time.

The following set of scans (parameters are listed in table 3.1) constituted one imaging period. While the longitudinal study only had only one period per animal the probe study had multiple periods depending on the drugs that were infused.

Scan 1) Localizer scan to check animal position and track the position of kidneys.

Scan 2) Anatomical imaging ( $\mathrm{T}_{2}$-weighted): Using a fat suppressed 3-dimensional volumetric interpolated SPGR sequences, in the axial plane.

Scan 3) Anatomical imaging ( $\mathrm{T}_{1}$-weighted): Using a 3D fast spin echo (3D-FSE ).

Scan 4) Measurement of oxygenation and microperfusion of the renal parenchyma: Using mGRE BOLD-MRI to measure $\mathrm{T}_{2}{ }^{*}$ and inflow effects.

Scan 5) Obtain anatomical FSE images at same spatial resolution as mGRE.

Scan 6) Measurement of renal perfusion using FAIR ASL-MRI.

Scan 7) Obtain FSE anatomical images at same spatial resolution as ASL.

Scan 8) Measurement of $\mathrm{T}_{1}$ relaxation constant using axial spin-echo inversion recovery sequence. 
Table 3.1: MRI parameters for the MRI scans.

\begin{tabular}{|c|c|}
\hline MRI Scan & Imaging Parameters \\
\hline $\begin{array}{l}\text { 1) Plane } \\
\text { Localizer }\end{array}$ & $\begin{array}{l}\text { Matrix: } 256 \text { x 128, NEX: 1, FOV: } 15 \mathrm{~mm} \text {, Slice Thickness: } 2 \mathrm{~mm} \\
\text { \# Slices: } 17 \text {, Slice spacing: } 0 \mathrm{~mm}\end{array}$ \\
\hline 2) $3 \mathrm{D}-\mathrm{SPGR}$ & $\begin{array}{l}\text { Matrix: } 256 \text { x 192, NEX: 8, FOV: } 12 \mathrm{~mm}, \mathrm{ST}: 1.6 \mathrm{~mm}, \mathrm{BW}: 31.25 \mathrm{KHz} \text {, } \\
\text { TE: } 1.7 \mathrm{~ms}, \text { FA: 10, Locations/Slab: } 32\end{array}$ \\
\hline 3) 3D-FSE & $\begin{array}{l}\text { Matrix: } 256 \text { x 192, NEX: 1, FOV: } 12 \mathrm{~mm}, \mathrm{ST}: 1.5 \mathrm{~mm}, \mathrm{BW}: 15.63 \mathrm{KHz}, \\
\text { TE: } 68 \mathrm{~ms} \text {, TR: } 3000 \mathrm{~ms} \text {, ETL: 32, Locations/Slab: } 20\end{array}$ \\
\hline 4) $2 \mathrm{D}-\mathrm{mGRE}$ & $\begin{array}{l}\text { Matrix: } 256 \text { x 192, NEX: 6, FOV: } 12 \mathrm{~mm}, \mathrm{ST}: 3 \mathrm{~mm}, \mathrm{BW}: 31.25 \mathrm{KHz} \\
\text { TE: } 4.2 \text { to } 35.2 \text { with increments of } 3.88 \mathrm{~ms} \text {, TR: } 100 \mathrm{~ms} \text {, FA: } 30 \text {, \# Slices: } 5\end{array}$ \\
\hline 5) $\mathrm{FSE}-12 \mathrm{~cm}$ & $\begin{array}{l}\text { Matrix: } 256 \text { x 192, NEX: 2, FOV: } 12 \mathrm{~mm}, \mathrm{ST}: 3 \mathrm{~mm} \text {, } \\
\text { TE: } 68 \mathrm{~ms} \text {, TR: } 2000 \mathrm{~ms} \text {, ETL: 8, \# Slices: 5, Slice spacing: Interleaving. }\end{array}$ \\
\hline 6) $\mathrm{ASL}$ & $\begin{array}{l}\text { Matrix: } 96 \text { x 128, FOV: } 15 \text { mm, ST: } 3 \text { mm, BW: } 100 \mathrm{KHz}, \\
\text { TE: } 10 \text { ms, TR: } 2500 \text { ms, FA: 90, \# Slices: 5, Slice spacing: Interleaving. }\end{array}$ \\
\hline 7) FSE- $15 \mathrm{~cm}$ & $\begin{array}{l}\text { Matrix: } 256 \text { x 192, NEX: 2, FOV: } 15 \mathrm{~mm} \text {, ST: } 3 \mathrm{~mm} \text {, } \\
\text { TE: } 68 \mathrm{~ms} \text {, TR: } 2000 \mathrm{~ms} \text {, ETL: 8, \# Slices: 5, Slice spacing: } \\
\text { Interleaving. }\end{array}$ \\
\hline $\begin{array}{l}\text { 8) } \mathrm{T}_{1} \\
\text { Measurement }\end{array}$ & $\begin{array}{l}\text { Matrix: } 256 \text { x 192, NEX: 2, FOV: } 12 \mathrm{~mm}, \mathrm{ST}: 1.6 \mathrm{~mm}, \mathrm{BW}: 62.50 \mathrm{KHz}, \\
\text { TE: } 20 \mathrm{~ms} \text {, TR: } 3000 \mathrm{~ms} \text {, FA: 10, \# Slices: 5, TI: 350,400,450,500 ms. }\end{array}$ \\
\hline
\end{tabular}

\subsubsection{Animal Model}

Studies were performed on male Sprague-Dawley (SD) rats obtained from Harlan SD Inc. at 10 weeks of age. Animals $(\mathrm{N}=6)$ were called R1 to R6 for identification. Each animal condition, that is, their body weight, and date of birth were recorded before the experiments. The table 3.2 summarizes these parameters recorded at the start of the experiments. 
Table 3.2: Animal parameters recorded at the onset of the experiments.

\begin{tabular}{|c|c|c|c|}
\hline Animal & Date of Birth & Body weight (gm) & Phy. Condition \\
\hline R1 & $08 / 06 / 2004$ & 330 & Normal \\
\hline R2 & $08 / 06 / 2004$ & 304 & Normal \\
\hline R3 & $08 / 13 / 2004$ & 356 & Normal \\
\hline R4 & $08 / 13 / 2004$ & 337 & Normal \\
\hline R5 & $08 / 06 / 2004$ & 326 & Normal \\
\hline R6 & $08 / 06 / 2004$ & 332 & Normal \\
\hline
\end{tabular}

Animals R5 and R6 were injected with streptozotocin $(65 \mathrm{mg} / \mathrm{kg})$ in the intraperitoneal cavity to create an animal model of type 1 diabetes [33]. These animals develop high levels of blood glucose because the drug kills the islet cells in the pancreas that secrete insulin, thereby making the animal diabetic.

As the anesthetized (3-4\% isoflurane in 100\% O2 delivered via breathing mask) animal was placed in the scanner, remote monitoring was very important to maintain its physiological condition within normal limits [29]. As operator was in the control room and the animal inside the scanner, direct monitoring was not feasible. Temperature and motion tracking was used to monitor the animal throughout the experiment. All the connecting wires from the monitors to the animal were $\sim 30$ ' in length. Both these parameters played a crucial role in maintaining a steady-state condition of the animal.

\subsubsection{Temperature Measurements}

Temperature is an important physiological parameter. The rats are warm-blooded mammals but their efficiency to control the body temperature is poor compared to humans. Previous studies have shown that temperature has a major effect on the physiology of the animal. As the heart rate is slowed down by anesthesia, the ability to control temperature by altering circulation is impaired and so the animals were kept on a warming pad. Overall there was no more than $3^{\circ} \mathrm{C}$ change in temperature, which eliminated the need for temperature compensation for the $\mathrm{pO}_{2}$ measurements. 
The following steps were followed during the experiment:

1. Rectal temperatures were taken for each individual animal during the baseline scanning and its average core body temperature was noted. Real-time temperature was monitored and recorded manually from the control room.

2. If the animal's body temperature was decreasing by more than $2 \mathrm{C}$, the heating pad was replaced with another warm one, without disturbing the setup. Also additional cotton was placed on the animal as a blanket.

3. If the recorded temperature rose was more than $2 \mathrm{C}$, then the fan (within the scanner) was switched on so the airflow could cool the animal.

The motion tracking fiber optical probes were placed on the animal chest. The MacLab data acquisition set-up connected to the Macintosh displayed the real-time animal-breathing rate.

\subsubsection{Animal Preparation \& Experimental Set-up}

The following steps were followed for all MRI studies

1. Anesthesia was induced by $3-4 \%$ isoflurane in $100 \% \mathrm{O}_{2}$ in a chamber and maintained at $2-3 \%$ in $100 \% \mathrm{O}_{2}$ supplied through a face mask at $11 / \mathrm{min}$.

2. Rats were placed on an MRI compatible heating pad which was pre-heated to 37 ${ }^{\circ} \mathrm{C}$ in order to maintain the animal body temperature.

3. Core temperature was monitored rectally using a temperature probe (YSI, Precession Thermometer, Ohio) and was maintained at 36-38C.

4. Rats scanned for the longitudinal survival studies were placed on a specially made plastic support table with an attachment for the immobilization/isolation cup. The left kidney was then immobilized using the plastic cup with a lateral cutout placed to ensure unobstructed flow through the renal artery.

Note: These additional steps were taken for the non-survival combined MRI- probe studies: 
5. A small incision was made for catheterization of the right femoral artery (for mean arterial pressure) and vein (for infusion of inulin, PAH, furosemide and Angiotensin II). The infusion pump was located in the control room.

6. Mean arterial pressure (MAP) from a catheter inserted in the femoral artery was monitored using a pressure transducer (Baxter), calibrated with a sphygmomanometer. Polyethylene tubing (Intramedic, dia. 0.05 ") of $10 \mathrm{~m}$ length was used to connect the rat femoral artery in the scanner to the pressure transducer located in the control room.

7. The abdomen was opened through a small, suprapubic midline incision, and the bladder was catheterized with a flanged PE90 catheter for urine collection. Urine was collected between periods in the scanner.

8. The rat was then placed on a specially made plastic support table to facilitate blood and urine collections, adapted from that described by [Ries, 2003 \#238].

9. For measurement of regional blood flow and tissue oxygenation, bare fiber probes $(\mathrm{BF} / \mathrm{OF})$ with tip $\mathrm{OD}=480 \mu\left(\mathrm{OxyFlo}^{\mathrm{TM}}\right.$ instruments, Oxford Optronix, Oxford, UK) were inserted into the renal cortex (1.5 $\mathrm{mm}$ in depth) and renal inner medulla (5.5 $\mathrm{mm}$ in depth).

10. The left kidney was then exposed and immobilized using a plastic cup with a lateral cutout for the renal artery.

11. The cup was attached to a plexiglass rod that had a bore to hold the dual $\mathrm{pO}_{2}$ /perfusion prob. Holders with different rod lengths were used to implant the probe in the medulla or cortex, as desired.

12. Saline-moistened gauze was placed over the open kidney. The fiber optic infra cable for motion tracking was placed on the animal chest.

13. The probe $\mathrm{pO}_{2}$, blood flow, MAP and motion measurements were recorded by a PowerLAB interface connected to a Power Macintosh G4.

After surgical preparation, the animal was maintained on the specially made plexiglas support table with a hollow base to hold a heated pad to maintain the animal core temperature of $36-38^{\circ} \mathrm{C}$. Rats were anesthetized with isoflurane and placed supine on the support table in the MRI coil, which is an open 7" diameter cylinder coil. All rats 
had oxygen saturation (\%StO2) monitored by a near-infrared system (Hutchinson) throughout MRI scanning.

\subsubsection{Longitudinal Study}

The set of longitudinal experiments only consisted of MR imaging and no surgery was performed. The rats were anesthetized for the entire $40 \mathrm{~min}$. scan with temperature and motion tracking. The following table indicates the different time points when the animals were imaged.

Table 3.3: Imaging time points and body weights.

\begin{tabular}{|c|c|c|c|c|c|c|}
\hline $\begin{array}{c}\text { Date/ } \\
\text { Animal }\end{array}$ & $\begin{array}{c}\text { R1 } \\
\text { Body Wt. /Age }\end{array}$ & $\begin{array}{c}\text { R2 } \\
\text { Body Wt. /Age }\end{array}$ & $\begin{array}{c}\text { R3 } \\
\text { Body Wt. /Age }\end{array}$ & $\begin{array}{c}\text { R4 } \\
\text { Body Wt. /Age }\end{array}$ & $\begin{array}{c}\text { R5 } \\
\text { Body Wt. /Age }\end{array}$ & $\begin{array}{c}\text { R6 } \\
\text { Body Wt. /Age }\end{array}$ \\
\hline $\mathbf{1 0} / \mathbf{1 1} / 04$ & $330 \mathrm{~g} / 66$ days & $337 \mathrm{~g} / 66$ days & - & - & $326 \mathrm{~g} / 66$ days & $340 \mathrm{~g} / 66 \mathrm{days}$ \\
\hline $\mathbf{1 0} / \mathbf{2 0} / \mathbf{0 4}$ & $359 \mathrm{~g} / 75$ days & $385 \mathrm{~g} / 75$ days & - & - & $328 \mathrm{~g} / 75$ days & $340 \mathrm{~g} / 75 \mathrm{days}$ \\
\hline $\mathbf{1 0} / \mathbf{2 3} / \mathbf{2 0 0 4}$ & - & - & - & - & - & $330 \mathrm{~g} / 78 \mathrm{days}$ \\
\hline $\mathbf{1 0} / \mathbf{2 5} / \mathbf{2 0 0 4}$ & - & - & - & - & $330 \mathrm{~g} / 80$ days & - \\
\hline $\mathbf{1 0} / \mathbf{2 7} / \mathbf{0 4}$ & $354 \mathrm{~g} / 82$ days & $389 \mathrm{~g} / 82$ days & - & - & - & - \\
\hline $\mathbf{1 0} / \mathbf{2 9} / \mathbf{0 4}$ & - & $410 \mathrm{~g} / 123$ days & $304 \mathrm{~g} / 77$ days & $356 \mathrm{~g} / 77$ days & - & - \\
\hline $\mathbf{1 2 / 0 8} / \mathbf{0 4}$ & $452 \mathrm{~g} / 130$ days & - & $400 \mathrm{~g} / 116$ days & $441 \mathrm{~g} / 116$ days & - & - \\
\hline $\mathbf{1 2} / \mathbf{1 5} / \mathbf{0 4}$ & - & - & $398 \mathrm{~g} / 123$ days & $440 \mathrm{~g} / 123$ days & - & - \\
\hline $\mathbf{0 3 / 2 2} / \mathbf{0 5}$ & - & - & $492 \mathrm{~g} / 220$ days & & - & - \\
\hline $\mathbf{0 3 / 2 2} / \mathbf{0 5}$ & - & - & - & $529 \mathrm{~g} / 222$ days & - & - \\
\hline
\end{tabular}

\subsubsection{MRI-Probe Study}

After the surgery, the animal was placed in the scanner. Real-time monitoring of MAP and temperature are done all through the experiment. MAP is known to be particularly sensitive to the changes in the animal with caused by the infusion of angiotensin-II and furosemide. The MAP obtained from each animal was recorded in the MacLab data acquisition software. After the experiment, the kidneys were harvested and sectioned to detect the location of the probe tip. The table 3.4 indicates the recorded parameters at the start of the experiment for each animal. 
Table 3.4: Imaging time points and body weights.

\begin{tabular}{|c|c|c|c|c|c|c|}
\hline & R1 & R2 & R3 & R4 & R5 & R6 \\
\hline Date & $12 / 08 / 04$ & $10 / 29 / 04$ & $03 / 22 / 05$ & $03 / 22 / 05$ & $10 / 25 / 04$ & $10 / 23 / 04$ \\
\hline Age (Days) & 130 & 123 & 220 & 222 & 80 & 78 \\
\hline Wt. (gm.) & 452 & 410 & 492 & 529 & 330 & 330 \\
\hline
\end{tabular}

Drugs were infused during imaging to observe variations in the oxygenation and the blood flow. The imaging protocol consisted of three periods: Angiotensin-II $(0.5 \mathrm{ml} / 100 \mathrm{gm} / \mathrm{min})$, control (saline) and furosemide $(10 \mathrm{mg} / \mathrm{ml})$ infusions. In previous studies, angiotensin II is know to show a decrease of both cortical and medulary oxygenation, while furosemide shows an increase in kidney oxygenation. Also, furosemide greatly reduces the blood flow in the cortical region, while aniotensin II slightly increases blood low in the medulla.

\subsubsection{Probe Placement}

Care was taken to insert the probe in the medulla. This was done by inserting the probe to a specific depth into the kidney. During MRI, the images from FSE pulse sequence were used to locate the probe inside the kidney. The slice with the probe was avoided for comparison with the mGRE image in order to avoid susceptibility artifact from the probe.

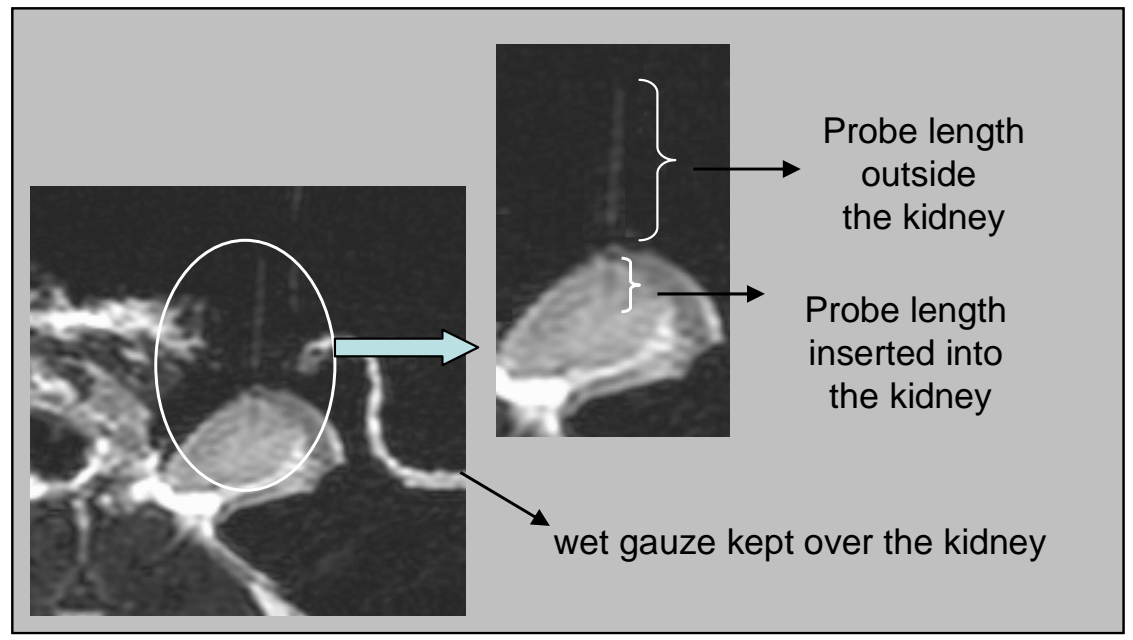

Figure 3.5: Image showing the probe position. 


\subsection{Data Analysis}

\subsubsection{MRI-Oxygenation Data Analysis}

The following steps were followed to obtain the $\mathrm{T}_{2} *$ values from the data obtained by imaging.

1. An FSE $(\mathrm{FOV}=12 \mathrm{~mm})$ pulse sequence generated a set of images for the same 5 prescribed slices used in the all the scans.

2. The mGRE pulse sequence generated a set of 8 images for each of the 5 slice across the kidney.

3. A Matlab script was used to generate the $\mathrm{T}_{2}{ }^{*}$-weighted images. The script computed the coefficient of a linear regression of the natural logarithm of the MR signal versus the echo time. This measurement represented the $\mathrm{R}_{2} *$ and its inverse generated the $\mathrm{T}_{2}{ }^{*}$ values. Also intercept and error maps were generated for each slice.

4. For analyzing the data, the MRVision image analysis software package (MRVision, Inc. Boston MA) was used. The $\mathrm{T}_{2}{ }^{*}$ images were loaded into the software along with the FSE image of that particular slice with the same field of view $(12 \mathrm{~mm})$. The later echo $\mathrm{T}_{2}{ }^{*}$-weighted image has relatively less signal as compared to the earlier echoes. For properly identifying the exact location of the various anatomical regions of the kidney, a region of interest (ROI) was selected on the FSE image. Then it was verified that the ROI was not over a region affected by susceptibility artifact on the later echo mGRE images as such an ROI would give an incorrectly short $\mathrm{T}_{2} *$. The same ROI was then put on the $\mathrm{T}_{2}{ }^{*}$ image and was integrated over the ROI to measure the mean $\mathrm{T}_{2} *$. In this way mean $\mathrm{T}_{2}{ }^{*}$ values for Anterior Cortex (AC), Posterior Cortex (PC) and Medulla were obtained.

5. The values from the three different sections of the kidney have been tabulated and plotted over time to observe the $\mathrm{T}_{2}{ }^{*}$ stability over time. [for the longitudinal study] 


\subsubsection{MRI-Perfusion Analysis}

Perfusion can be calculated from the subtraction of the tagged image from the control image using tested techniques [18] to calculate the blood flow in $\mathrm{ml}$ of blood per $\mathrm{ml}$ of tissue per min. Quantitative measures of perfusion were calculated using measurements of modified for kidney from procedures described by Wong [2].

The following steps were followed to obtain the perfusion values from the data obtained by imaging.

1. An FSE $(F O V=15 \mathrm{~cm})$ pulse sequence generated a set of images for the same 5 prescribed slices used in the all the scans.

2. $\mathrm{T}_{1}$ measurement scans generated image data sets for $\mathrm{TI}=350,400,450,500,800$, $1200 \mathrm{~ms}$. The images for each TI were closely observed to find the darkest possible kidney contrast, indicating its particular TI. This TI was used to compute $\mathrm{T}_{1}$ for the perfusion calculations.

3. The ASL pulse sequence generated 125 pairs of images (125 tagged and 125 controls) for each of the five slices.

4. A Matlab script (ref: Appendix-B) was used to obtain an average of 125 images for tagged and control images respectively, and these average images were subtracted to obtain the difference image which is perfusion weighted. Likewise five image slices were obtained after subtraction.

5. The MRVision image analysis software package was used to compare the selected ROI's from the FSE image and the difference image. The average signal intensity of the ROI is a quantitative measure of perfusion.

\subsubsection{Optical Probes}

Real-time data from the optical probe was displayed and recorded on the Macintosh. The time period was noted for the duration of mGRE and ASL imaging sequences. The data from FQ oximetry (\# points averaged=100) was averaged over this time point to obtain the mean and standard deviation. This value indicated the invasive 
measurement of oxygenation in rat kidney. Similarly the data from the LDF was processed for the perfusion measurement.

\subsubsection{Comparing the Invasive and Noninvasive Methods}

The relationships between invasive and non-invasive measurements of perfusion and oxygenation were examined by plotting the non-invasive variables against the invasive variables [29]. The correspondence was evaluated statistically using paired ttests. 


\section{CHAPTER 4: RESULTS}

\subsection{Physiological Monitoring}

\subsubsection{Temperature Measurements}

Core body temperature was successfully maintained at $37{ }^{\circ} \mathrm{C}$, and a variability of no more than $3^{\circ} \mathrm{C}$ was observed.

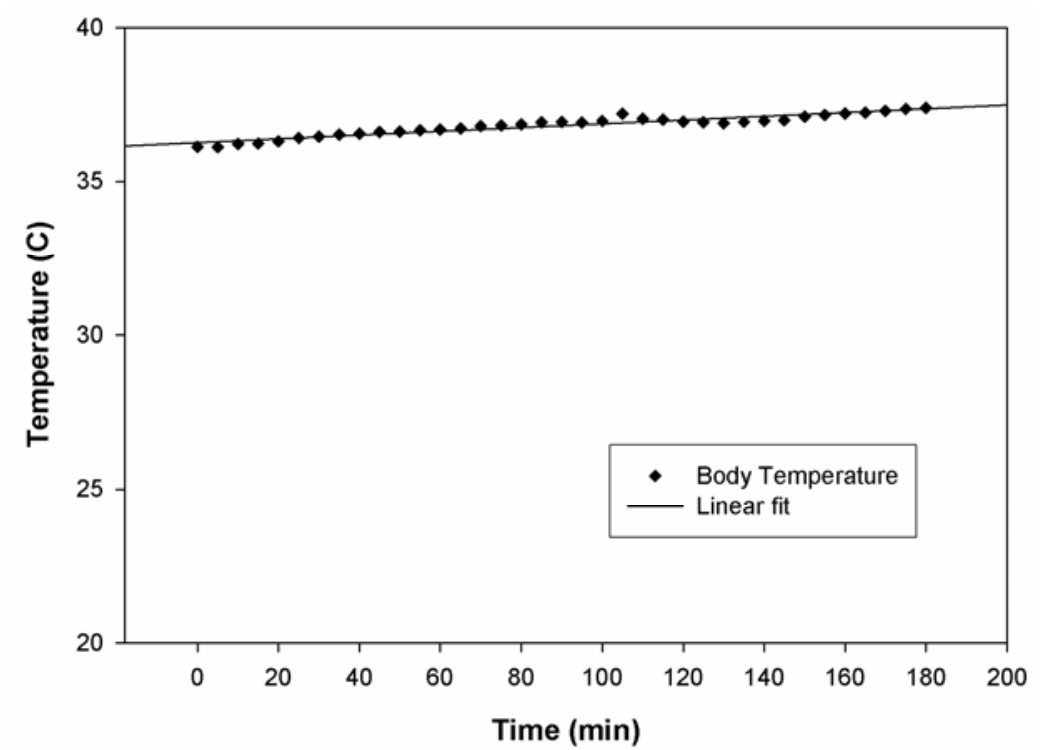

Figure 4.1: Plot indicating the variation of animal body temperature with respect to time.

The above plot indicates typical temperature variability over a period of 3 hours, monitored during combined probe study of R5. Temperature was controlled as described in section 3.3.3. In almost all of the experiments the heating pads proved to be were very effective for maintaining a relatively stable animal body temperature.

\subsubsection{Motion Tracking}

The motion detector was implemented successfully to monitor the respiratory motion of rats. The readings from the MacLab data acquisition software represented the chest movement of the rat, which is equivalent to the breathing motion of the animal. The peak excursion versus time could be used to calculate the respiration rate, which was a good real-time indicator and tracking parameter for the animal physiology. 


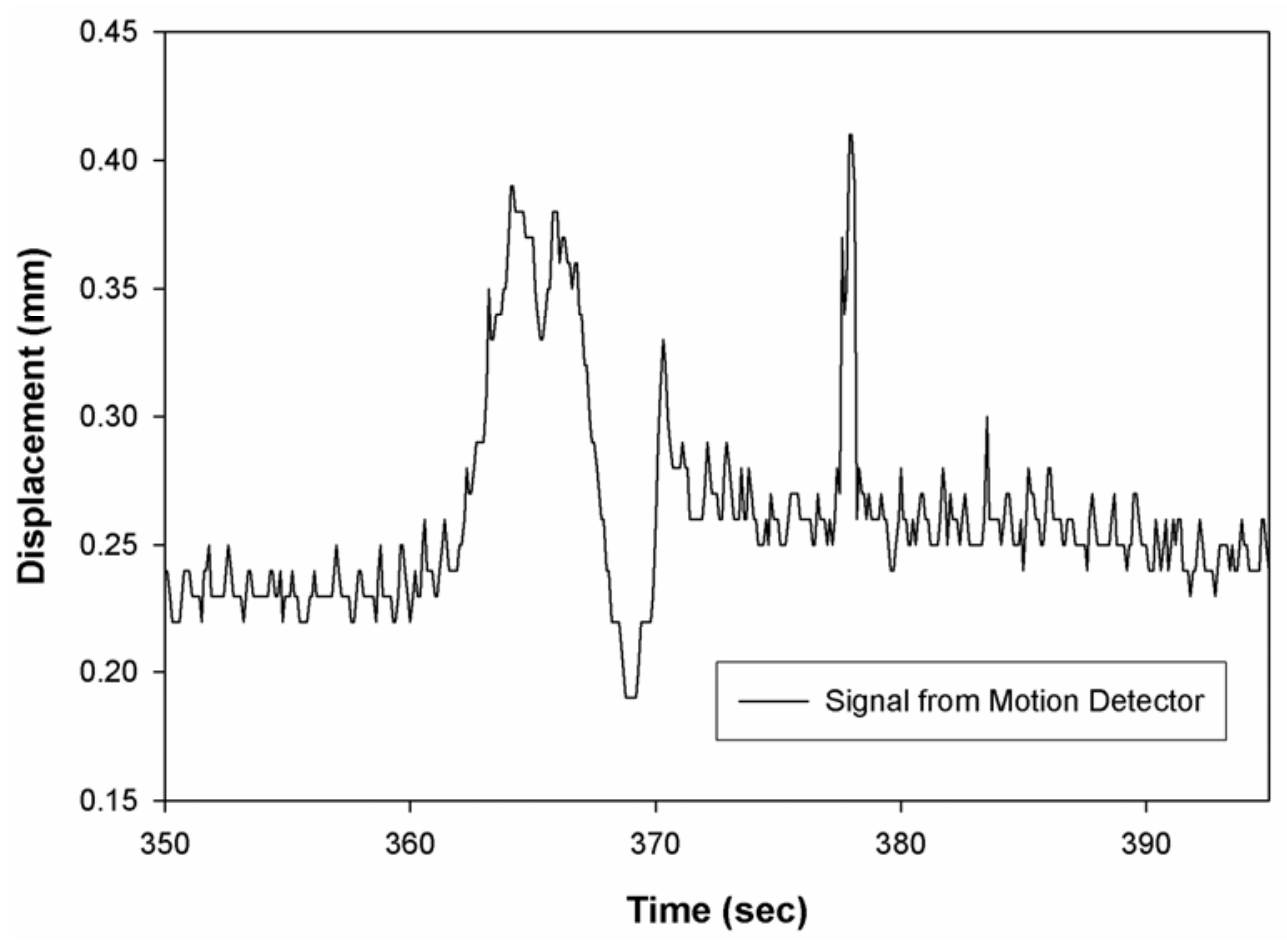

Figure 4.2: MacLab plot indicating animal movement.

In some early cases, when inactin was used as an anesthetic rather than isoflurane gas, accumulation of fluid in the lungs led to an increase in the breathing rate, thereby indicating a need for immediate attention to the animal. At times, if a sudden jerk was observed in the animal movement, that particular scan was discarded, the animal was checked and the same scan was repeated. In the figure4.2, the normal movement at 360$375 \mathrm{~s}$ is indicative of animal breathing rate and following sudden change in the displacement at $378 \mathrm{~s}$ is representative of animal movement.

The following sections of this chapter include the results and analysis of the longitudinal study, presented in section 4.2, while section 4.3 deals with the simultaneous MRI-probe study. In these sections, results will be discussed in detail for one representative animal. This will be followed by the collective representation of all the rats in the form of tables and plots 


\section{$\underline{4.2 \text { Longitudinal Study }}$}

\subsubsection{Oxygenation Results}

Images were acquired according to the imaging protocol presented in section 3.2.2. The images are presented below in figure 4.3 in the same order as they were acquired. Following are the images acquired from the animal R01.
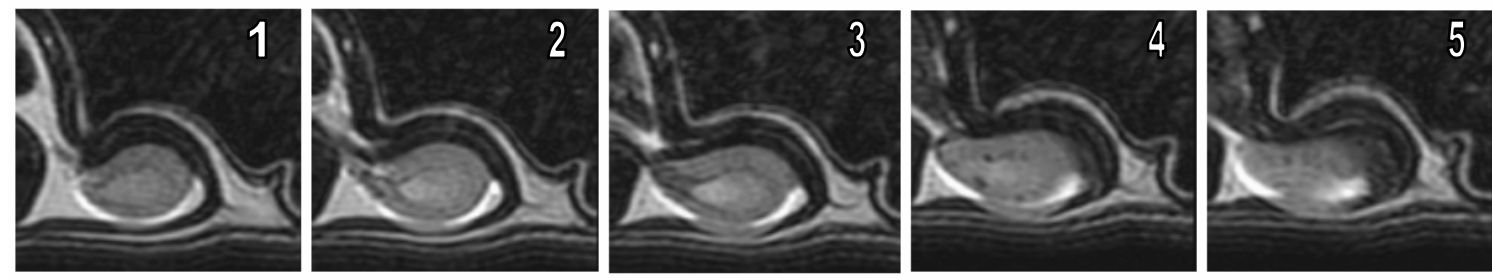

Figure 4.3: FSE images of 5 slices.

The above set of five slices indicates the axial sections of the rat kidney. The images are taken from inferior to superior along the animal body.

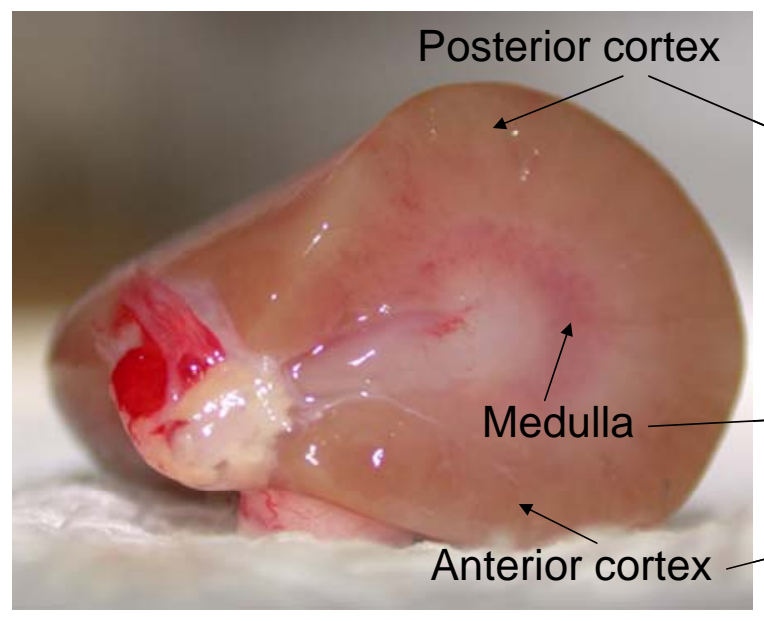

A photograph of a harvested kidney

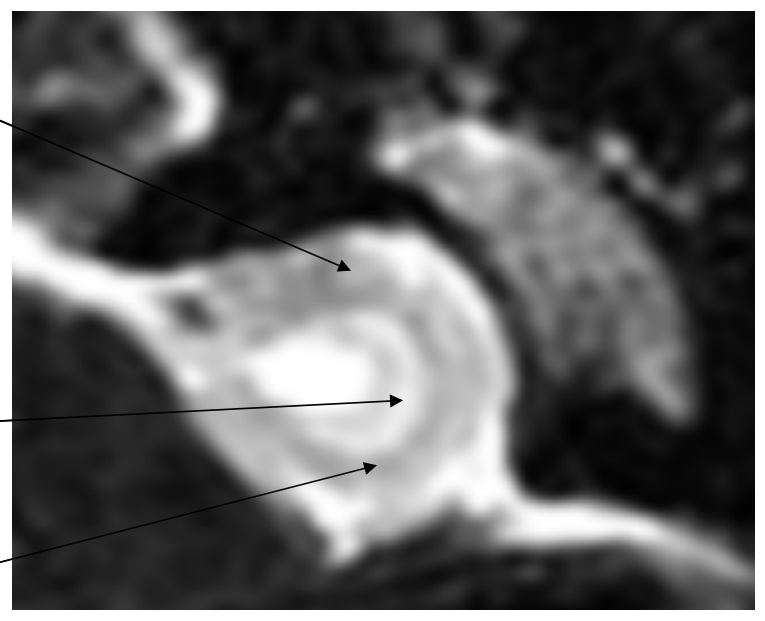

FSE image.

Figure 4.4: Understanding MRI-FSE image.

An FSE image of the kidney anatomy that shows the boundaries between the regions with higher contrast is obtained by FSE imaging. In the figure 4.4, the FSE image is compared with a harvested kidney. 

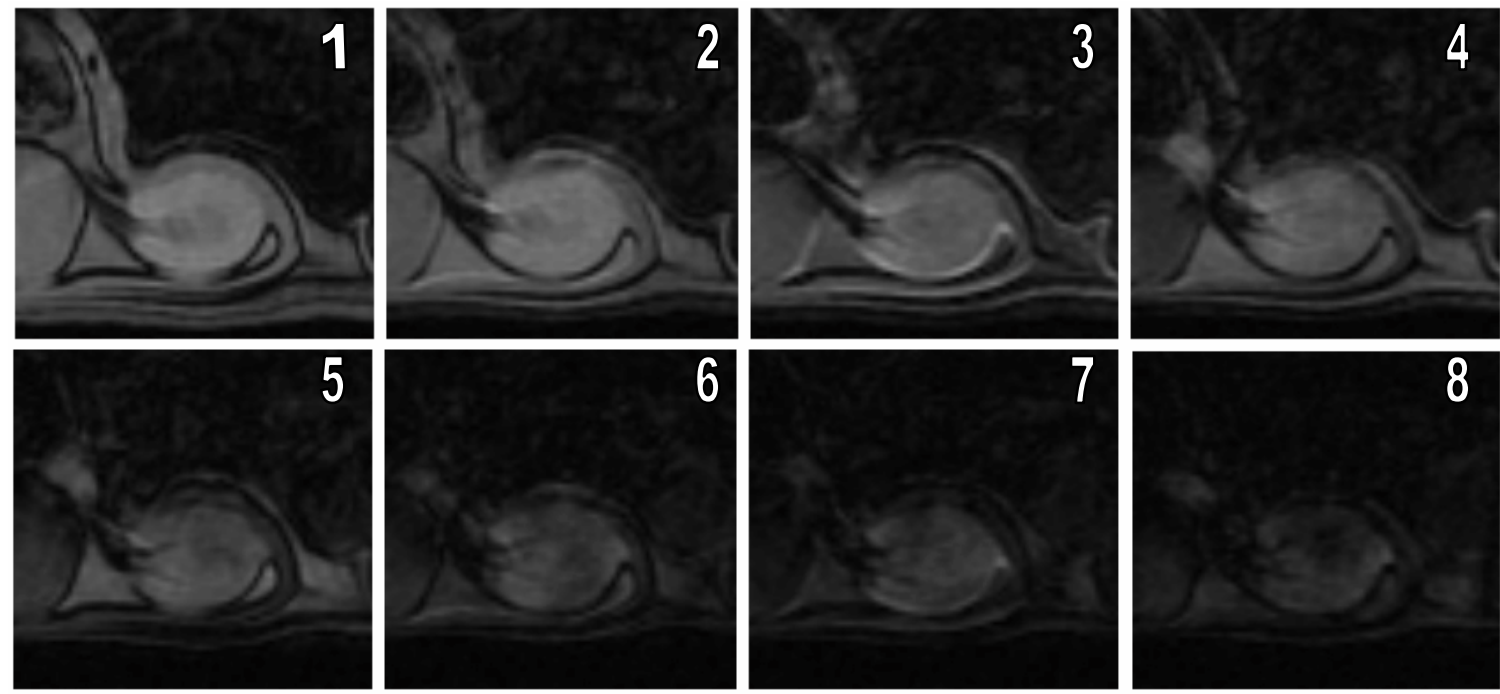

Figure 4.5: $m G R E$ image set with varying TE.

The above set of mGRE images are processed using Matlab (Version: 6.5, as described in section 3.3.1) to obtain the $\mathrm{T}_{2}{ }^{*}$-weighted image, the intercept and the error images. The error image is the residual value from the $1^{\text {st }}$ order polynomial fit routine used in Matlab.

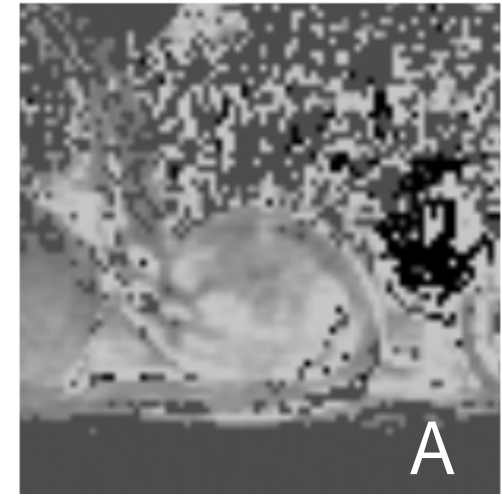

$\mathrm{T}_{2} *$ weighted image

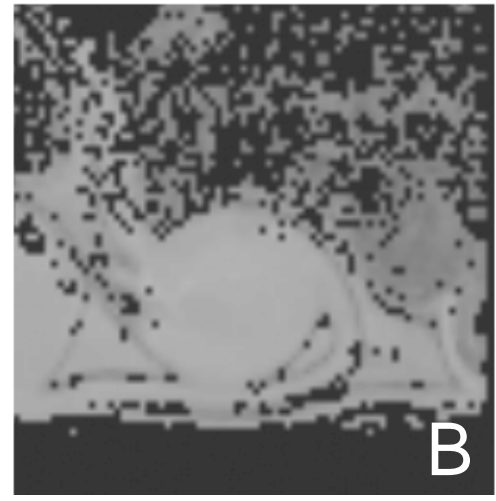

Intercept map

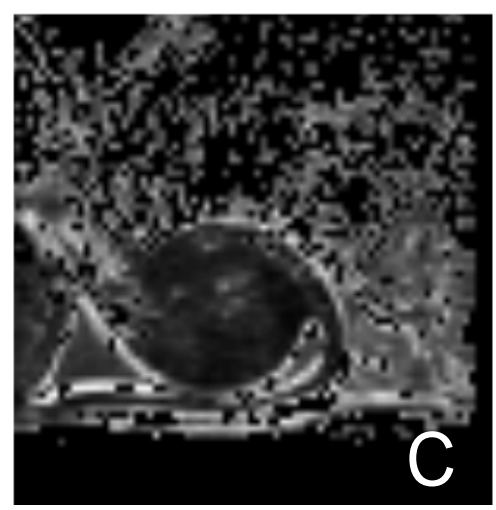

Error map

Figure 4.6: Post processed images.

A region of interest (ROI) is selected on the FSE image of the same slice and is copied on to the $\mathrm{T}_{2}{ }^{*}$-weighted image to obtain the average $\mathrm{T}_{2}{ }^{*}$ values in the selected ROI's. 


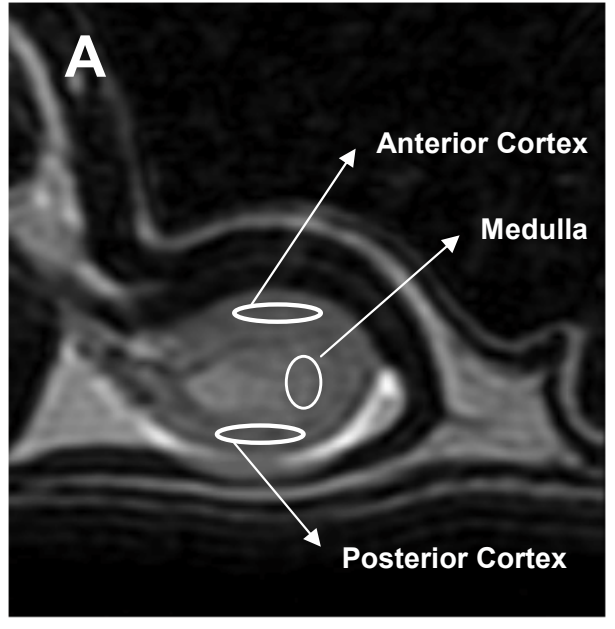

FSE image

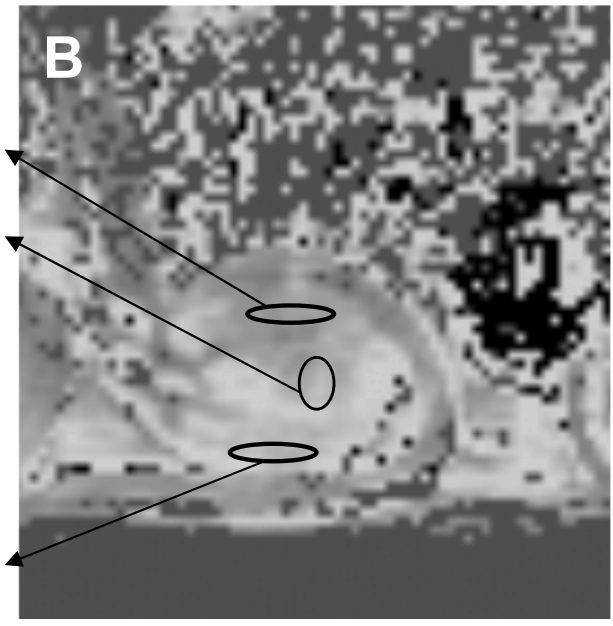

$\mathrm{T}_{2}{ }^{*}$ weighted image

Figure 4.7: ROI selection for $T_{2}{ }^{*}$ calculation.

$\mathrm{T}_{2} *$ values are obtained from the anterior cortex, posterior cortex and medulla of the kidney. These values are tabulated in table 4.1. The std. dev. was zero for value $<0.1$

Table 4.1: $T_{2}{ }^{*}$ values of selected ROI's in the kidney

\begin{tabular}{|c|c|c|c|c|c|c|c|}
\hline \multirow[t]{2}{*}{ Animal } & \multirow[b]{2}{*}{ Days } & \multicolumn{2}{|c|}{ Anterior cortex } & \multicolumn{2}{|c|}{$\begin{array}{c}\text { Posterior } \\
\text { Cortex }\end{array}$} & \multicolumn{2}{|c|}{ Medulla } \\
\hline & & Mean & Std.Dev & Mean & Std. Dev & Mean & Std. Dev \\
\hline \multirow{4}{*}{$\begin{array}{l}\text { Normal } \\
\text { ( R01) }\end{array}$} & 75 & 21.5 & 3.8 & 32.7 & 0 & 30.9 & 2.6 \\
\hline & 82 & 20.3 & 1.6 & 32.4 & 0.7 & 29.9 & 2.5 \\
\hline & 123 & 18.2 & 2 & 32.7 & 0.2 & 26.7 & 3.1 \\
\hline & 130 & 22.6 & 2.8 & 32.7 & 0.1 & 28.8 & 2.9 \\
\hline \multirow{4}{*}{$\begin{array}{l}\text { Normal } \\
\text { ( R02) }\end{array}$} & 66 & 17.7 & 6.5 & 31.4 & 2.4 & 28.5 & 4.6 \\
\hline & 75 & 22.8 & 5.6 & 31.5 & 1.8 & 29.7 & 3.1 \\
\hline & 82 & 16 & 1.4 & 31.8 & 1.8 & 28.2 & 1.3 \\
\hline & 84 & 20.1 & 1.1 & 31 & 1 & 28.3 & 2.2 \\
\hline \multirow{3}{*}{$\begin{array}{l}\text { Normal } \\
\text { ( R03 ) }\end{array}$} & 116 & 17.3 & 3.2 & 31.3 & 1.2 & 31.9 & 1.2 \\
\hline & 123 & 14.1 & 1.6 & 31.6 & 0.8 & 30.2 & 2 \\
\hline & 220 & 12.3 & 6 & 31.1 & 2.2 & 29.5 & 4.6 \\
\hline \multirow{3}{*}{$\begin{array}{l}\text { Normal } \\
\text { ( R04) }\end{array}$} & 116 & 17.2 & 2.7 & 31.1 & 1.5 & 24 & 1.6 \\
\hline & 123 & 16.1 & 1.2 & 31.4 & 1 & 26.3 & 2.3 \\
\hline & 222 & 19.2 & 4.8 & 30.1 & 2.1 & 22.8 & 0.8 \\
\hline \multirow{3}{*}{$\begin{array}{l}\text { Diabetic } \\
\text { ( R05 ) }\end{array}$} & 66 & 19.8 & 3.2 & 32.7 & 0 & 27.7 & 3.8 \\
\hline & 75 & 15.8 & 1.9 & 31.7 & 1.5 & 30.7 & 1.6 \\
\hline & 80 & 22.3 & 4.6 & 31.3 & 2.1 & 27.9 & 4.9 \\
\hline \multirow{3}{*}{$\begin{array}{l}\text { Diabetic } \\
\text { ( R } 06 \text { ) }\end{array}$} & 66 & 17.4 & 3 & 32.7 & 0 & 31 & 3 \\
\hline & 75 & 20.3 & 3.2 & 31.4 & 1.1 & 29.9 & 3.5 \\
\hline & 78 & 17.8 & 3.2 & 32.1 & 1.4 & 30.2 & 2.7 \\
\hline
\end{tabular}


The $\mathrm{T}_{2} *$ values of posterior cortex, anterior cortex and medulla have been plotted against the animal age in the following figures:

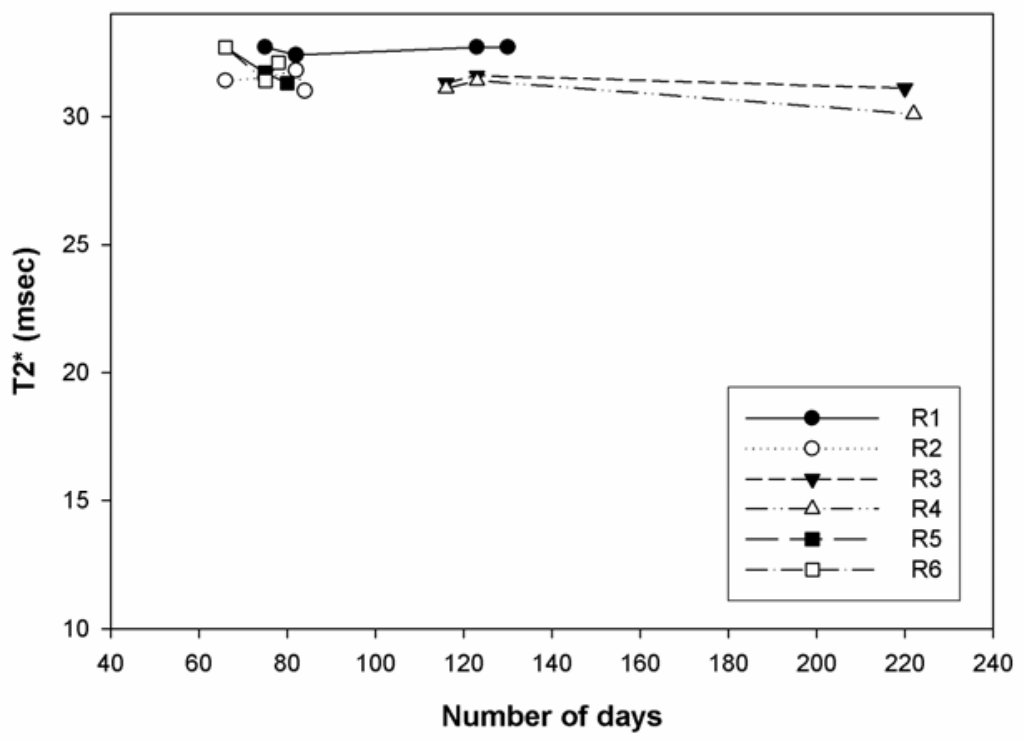

Figure 4.8: Plot indicating the changes in posterior cortex $T_{2}{ }^{*}$ with respect to time.

The $\mathrm{T}_{2}{ }^{*}$ values in the posterior cortex were observed to be consistent over time and independent of age. The average $\mathrm{T}_{2} *$ value observed was $31.83 \mathrm{msec}$ with a standard deviation of $0.7 \mathrm{msec}$. A relative deviation of less than $2.2 \%$ was observed over the entire set. The $\mathrm{T}_{2} *$ values in the two diabetic animals, R05 and R06 were also consistent.

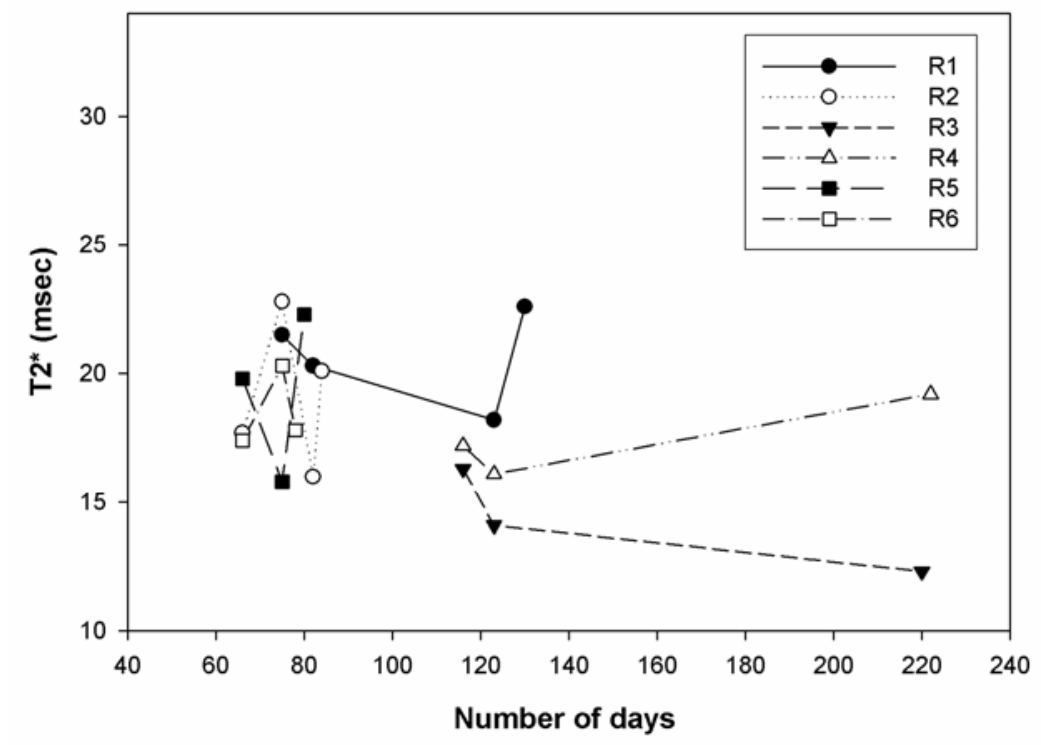

Figure 4.9: Plot indicating the changes in anterior cortex $T_{2}{ }^{*}$ with respect to time. 
The $\mathrm{T}_{2}{ }^{*}$ values in the anterior cortex demonstrate less consistency than in the posterior cortex. A mean value of $18.44 \mathrm{msec}$, with a standard deviation of $2.83 \mathrm{msec}$ was observed. This represents a $15 \%$ relative variation in the measurements. The $\mathrm{T}_{2} *$ values for the diabetic rats was within $15 \%$ relative variation.

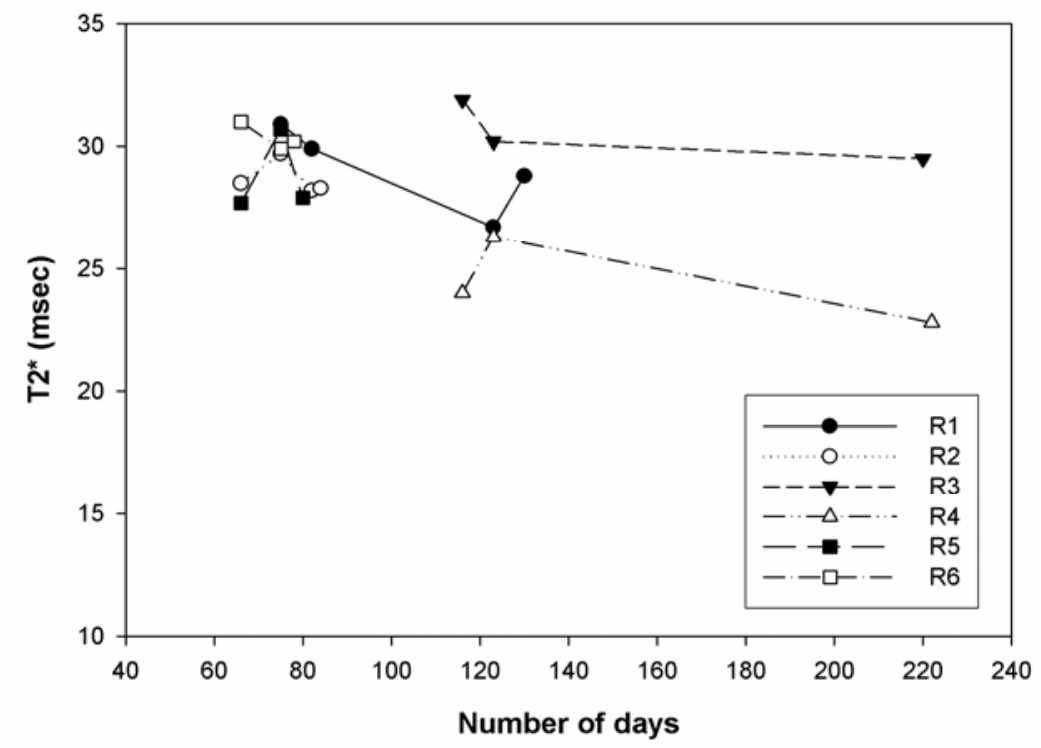

Figure 4.10: Plot indicating the changes in medulla $T_{2}{ }^{*}$ with respect to time.

The values in the medulla were more consistent than those in the anterior cortex. A mean value of $28.65 \mathrm{msec}$ was observed with a standard deviation of $2.31 \mathrm{msec}$ over the six animals. Overall, $8.1 \%$ relative variability in the $\mathrm{T}_{2}{ }^{*}$ values for all the animals was observed in the medulla.

\subsubsection{Arterial Spin Labeling}

ASL images were also acquired in the longitudinal study to observe the changes in perfusion values over time. The objective was to determine whether the perfusion values were consistent over time. The imaging protocols followed for acquiring the ASL images are mentioned in section 3.2.2. Following are the images acquired for ASL calculations for a control animal (R03). 


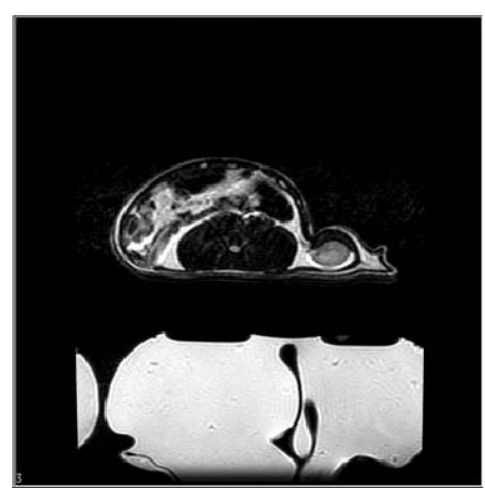

FSE image

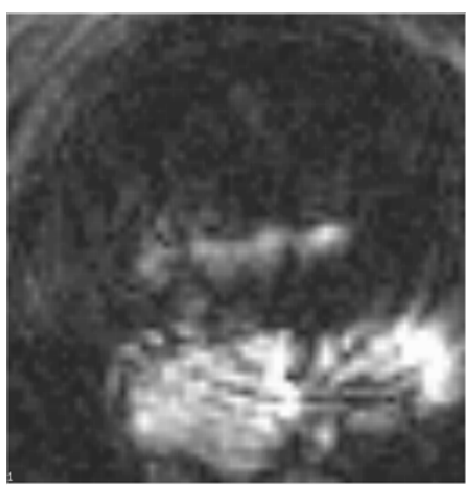

Tagged image

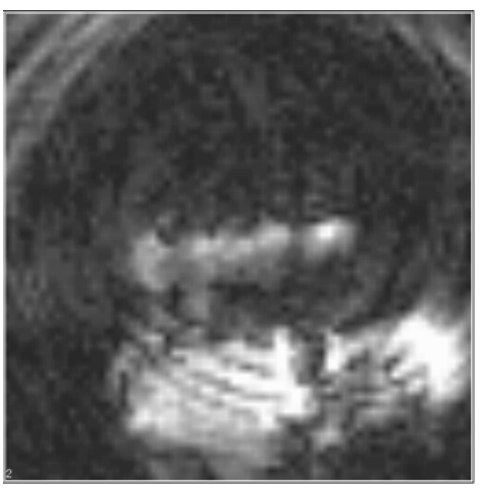

Control image

Figure 4.11: ASL image set.

The ASL images were acquired at a field of view (FOV) of $15 \mathrm{~cm}$, where as the mGRE images were acquired at a FOV of $12 \mathrm{~cm}$. More imaging area covered a large portion of the heating pad, which can be seen in the ASL tagged and the control images. Only small section of the animal body was visible in these images. The acquired ASL images are dominated by aliasing susceptibility artifact and blur.

The wrap around of the heating pad caused aliasing, as it was partially outside the FOV. As the image acquisition uses spiral trajectory for signal collection, the wrap around of the heating pad appears at the outer radius of the image. Blurring was also dominant in the images as the matrix size was low (64 X 64) when compared to the FSE ( $256 \mathrm{X} 256)$. The spatial resolution in the ASL images was $150 \mathrm{~mm} / 64=2.34 \mathrm{~mm}$.

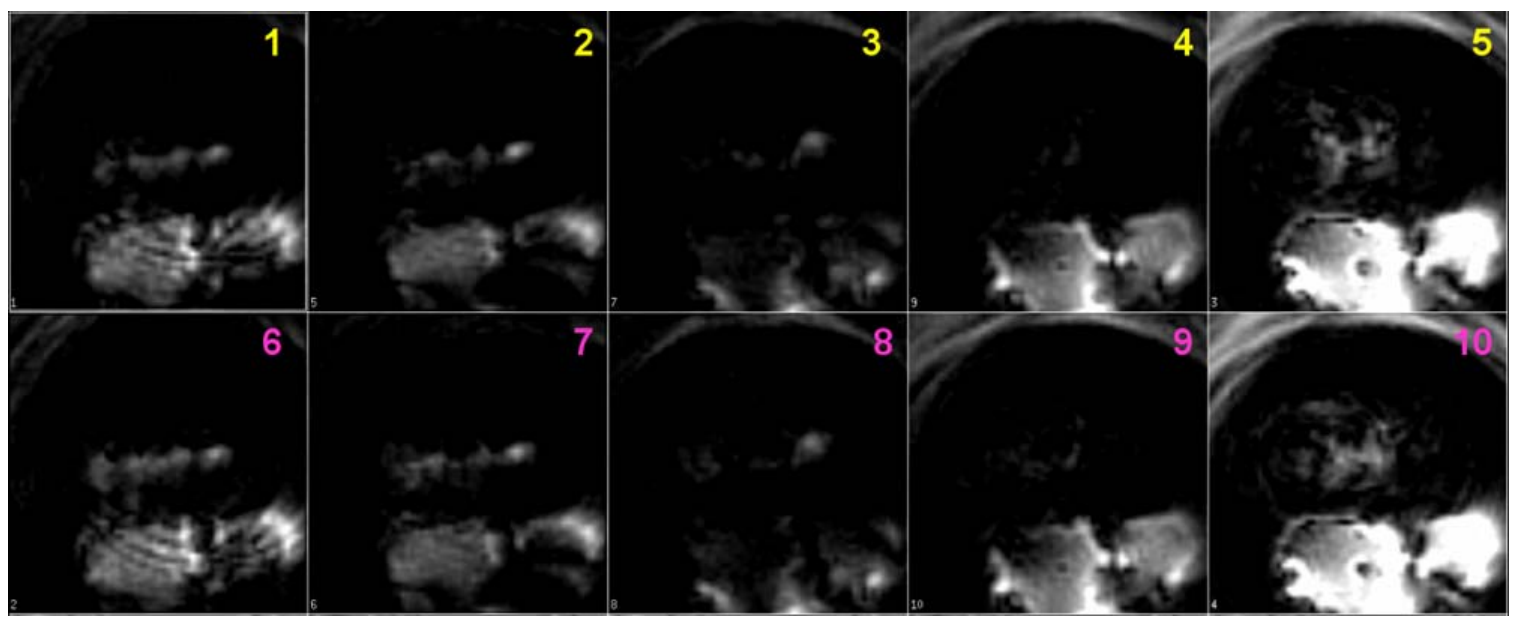

Figure 4.12: Tagged and control images of all 5 slices. 
Images 1-5 are averages of the 125 tagged images of the respective five slices and images 6-7 are averages of the 125 control images of same 5 slices.

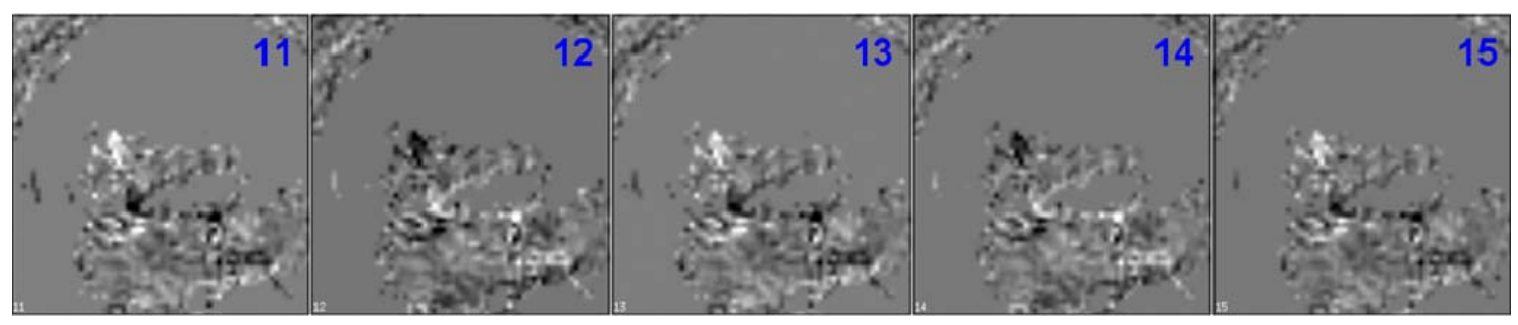

Figure 4.13: Perfusion weighted images obtained using Matlab processing.

Using the technique described in section 3.3.2, difference images for the 5 slices were generated using Matlab. Images 11-12 represent the perfusion weighted images calculated using equation 2.12 for the five slices. Only, a slight proportion of the rat body was visible in the perfusion image. The perfusion calculation was clearly problematic as there is no blood flow in the heating pad, but the heating pad was still seen in the perfusion image.

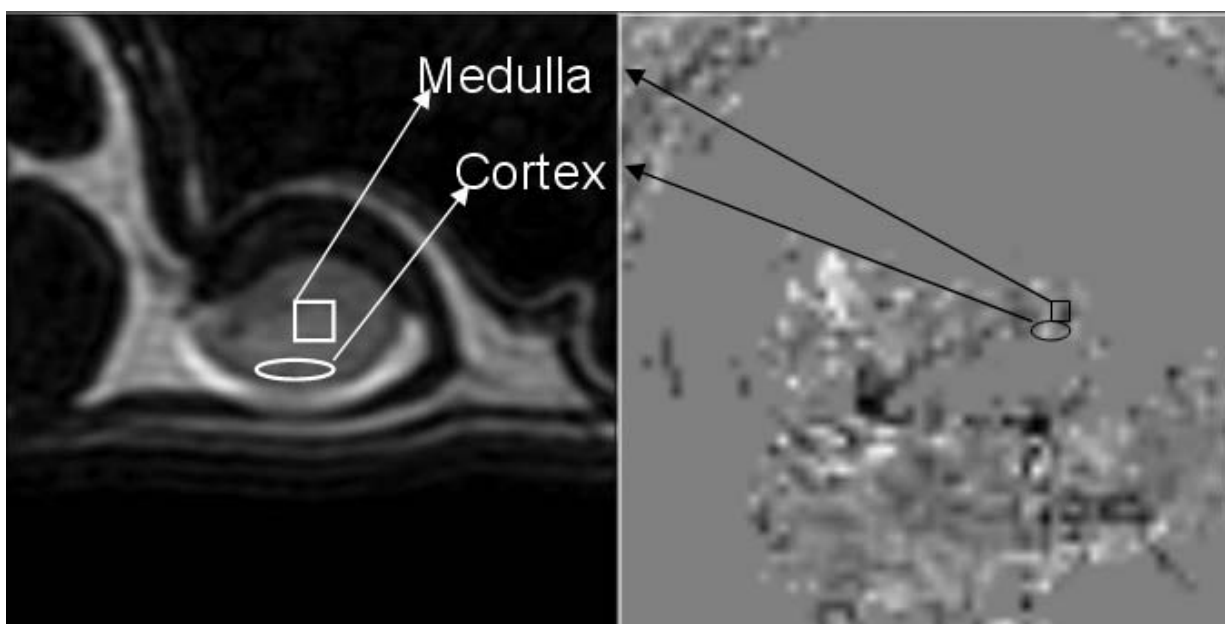

Figure 4.14: ROI selection for perfusion calculations.

The FSE and the perfusion weighted image were matched for selected ROI and values for perfusion in the regions were obtained. Not surprisingly, the values obtained were largely deviant from the values obtained by previous studies. An average of 0.2 $\mathrm{mL} / \mathrm{g} / \mathrm{min}$ was observed in the cortex, where as the previously reported perfusion values 
[17] indicated an average perfusion value of $4.9 \mathrm{~mL} / \mathrm{g} / \mathrm{min}$. The multiple problems encountered were:

1. Low matrix size

2. Low SNR

3. Dominance of blur and aliasing artifact

4. Tagging issues

An attempt was made to obtain good SNR by increasing the matrix size for animal R01. The matrix size was increased from 64 X 64 to $128 \times 128$, keeping the remaining parameters same.

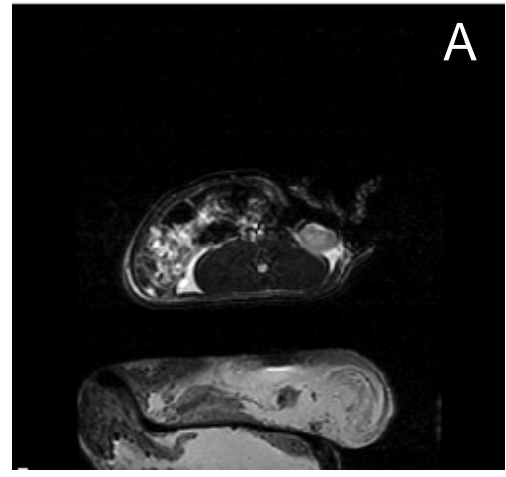

FSE image

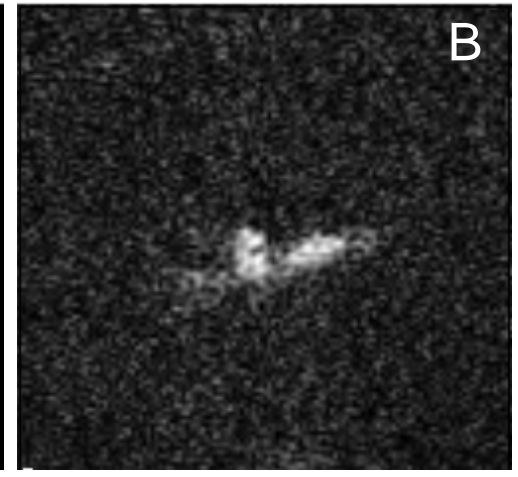

Tagged image

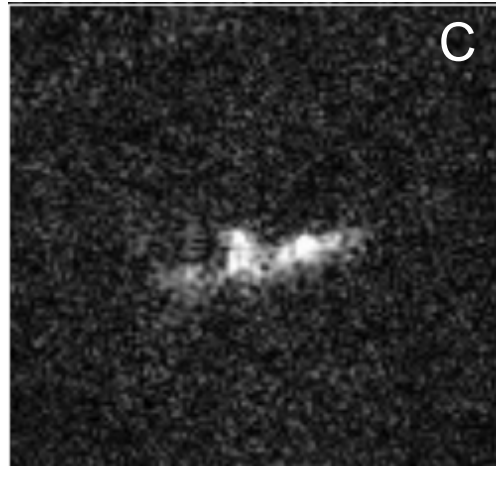

Control image

Figure 4.15: ASL image set for slice 1.

With the increase in the matrix size, the aliasing artifact was greatly reduced which can be observed in the figure 4.15. The perfusion values observed in the difference perfusion weighted image deviated from the standard values as at 64 X 64 .

Tagging was another possible issue, which was responsible for the signal loss in the image. A tag thickness of $25 \mathrm{~mm}$ was used for the ASL imaging. This may have been too low. 


\subsection{MRI-Probe Study}

As described in section 3.3.3, the probe study was carried out simultaneously with MRI measurements and with the invasive measurements using the implanted probe. This experiment had three periods as described. Physiological parameters such as temperature, motion and MAP readings were acquired on real-time basis, all through the experiment.

\subsubsection{Mean Arterial Pressure (MAP)}

The MAP obtained from each animal was recorded in the MacLab data acquisition software. A relative increase in MAP was observed during infusion of Angiotensin-II, for comparison to the control state. A noticeable decrease was observed with furosemide infusion compared to the control.

1. During the control period, the MAP was in the range of $80-115 \mathrm{mmHg}$. The variability was less than $10 \%$ from mean MAP $(111.33+/-13.82)$.

2. In the Angiotensin-II infusion period, there was a 30-40\% shoot up in the MAP. It took nearly $10-15 \mathrm{~min}$ after the infusion, to reach a stable reading. The mean MAP was observed to be $137.83+/-20.7$

3. During the furosemide infusion period, there was a noticeable decrease in the MAP. On an average 5-10\% decrease was observed from that of the control MAP reading. The mean MAP was observed to be $96+/-11.26$

Table 4.2: Table indicating the change of MAP at different time periods.

\begin{tabular}{|c|c|c|c|c|c|c|c|}
\hline $\begin{array}{c}\text { Time periods } \\
\text { / Animals }\end{array}$ & $\begin{array}{c}\text { R1 } \\
(\mathrm{mmHg})\end{array}$ & $\begin{array}{c}\mathbf{R 2} \\
(\mathrm{mmHg})\end{array}$ & $\begin{array}{c}\mathbf{R 3} \\
(\mathrm{mmHg})\end{array}$ & $\begin{array}{c}\mathbf{R 4} \\
(\mathrm{mmHg})\end{array}$ & $\begin{array}{c}\mathbf{R 5} \\
(\mathrm{mmHg})\end{array}$ & $\begin{array}{c}\text { R6 } \\
(\mathrm{mmHg})\end{array}$ & $\begin{array}{c}\text { Mean +/- } \\
\text { Std. Dev. }\end{array}$ \\
\hline AngiotensinII & 124 & 167 & 135 & 109 & 138 & 154 & $\begin{array}{c}137.83 \\
+/-20.7\end{array}$ \\
\hline Control & 109 & 122 & 114 & 85 & 116 & 122 & $\begin{array}{c}111.33 \\
+/-13.82\end{array}$ \\
\hline Furosemide & 103 & - & 102 & 83 & - & - & 96 \\
$+/-11.26$ \\
\hline
\end{tabular}




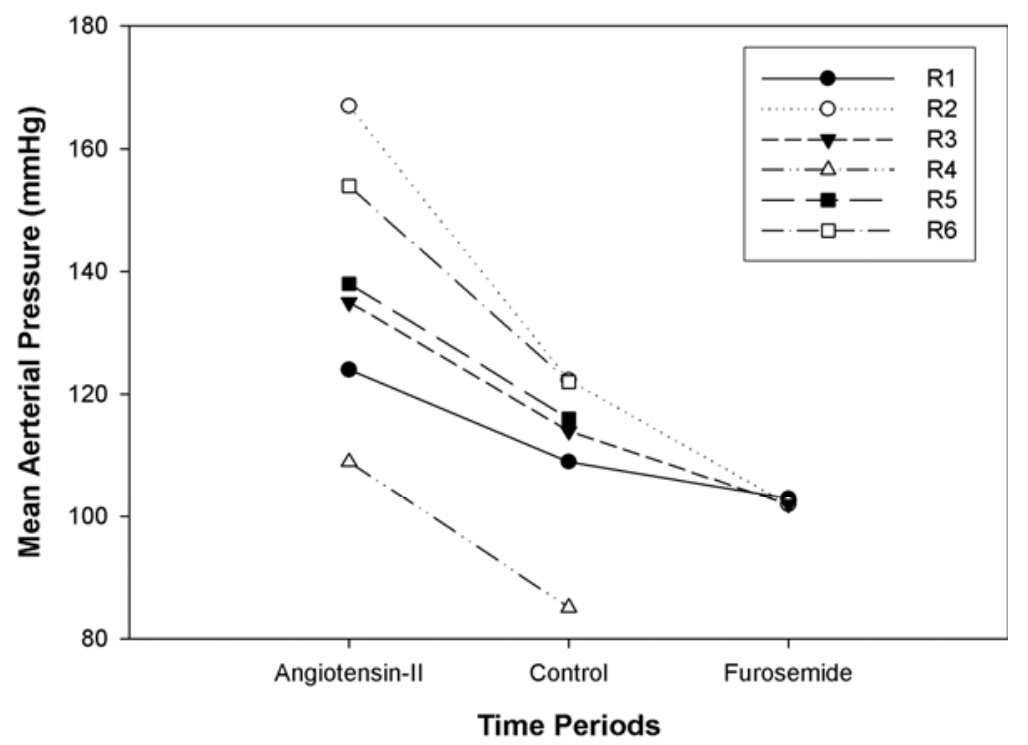

Figure 4.16: Plot indicating the changes in MAP with respect to the three time periods.

The MAP values were plotted verses time for individual animals. For the animals R04, R05, R06 furosemide infusion was not used. The increase in the MAP readings during Ang II with respect to the control, were observed. Also, the decrease of MAP with furosemide was observed. The diabetic animals R05 and R06 showed no different response for MAP compared to the remaining control animals.

\subsubsection{Oxygenation Data Results}

As described in section 3.3.1, the acquisition and processing steps were followed to obtain the $\mathrm{T}_{2}{ }^{*}$ values. Following are the image data set obtained from a normal animal.

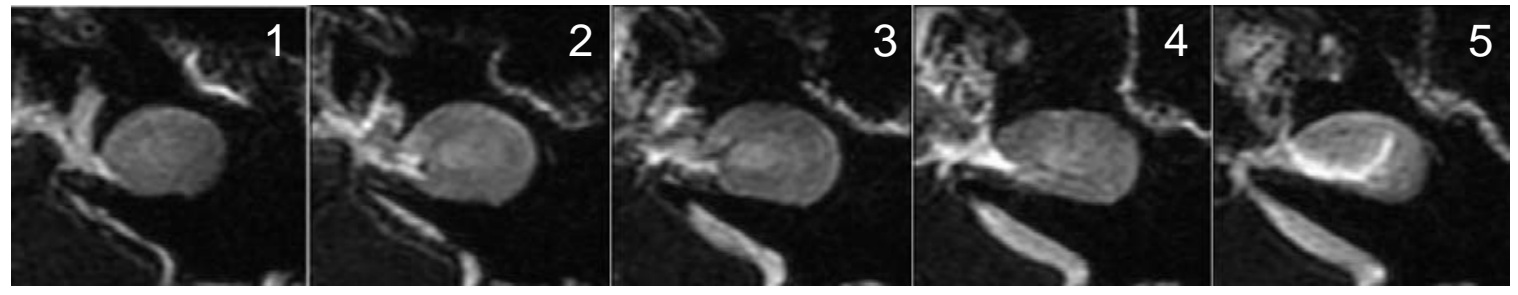

Figure 4.17: FSE images of all 5 slices. 


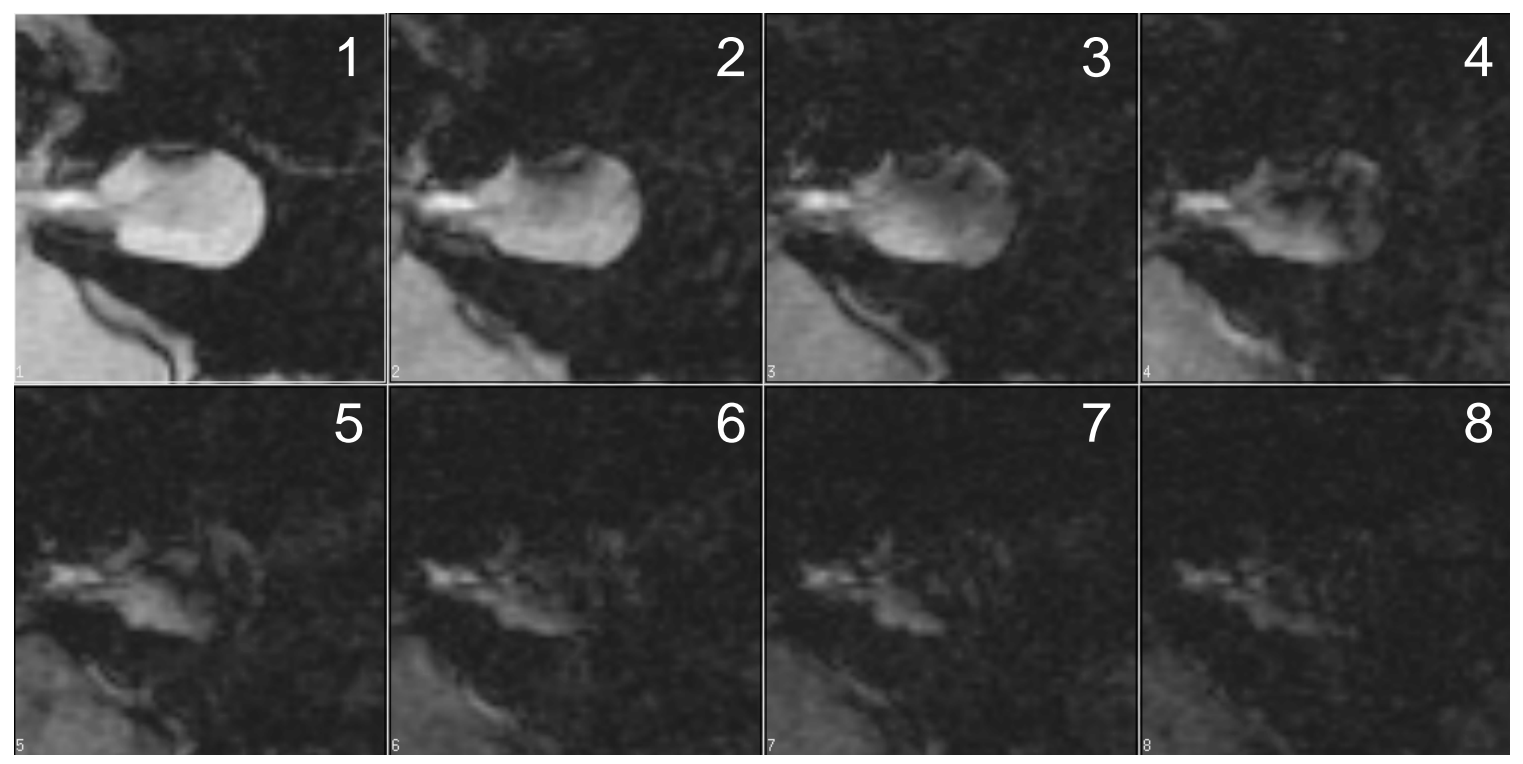

Figure 4.18: $m G R E$ image set.

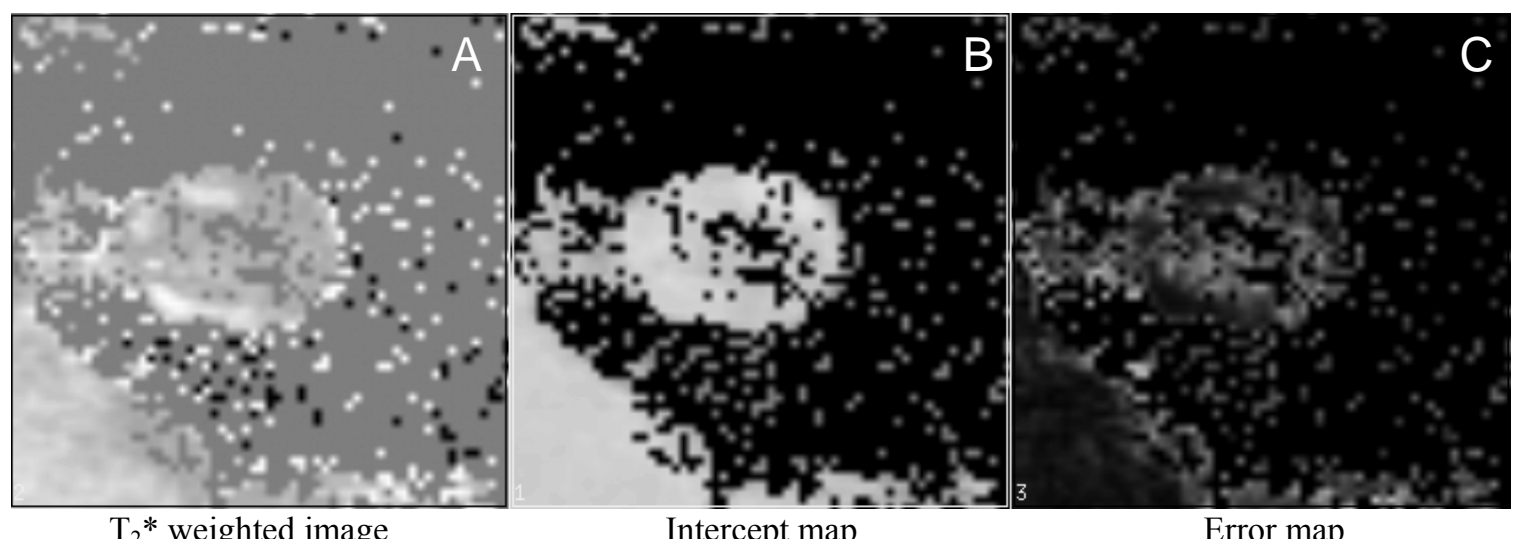

Figure 4.19: Post processed images.

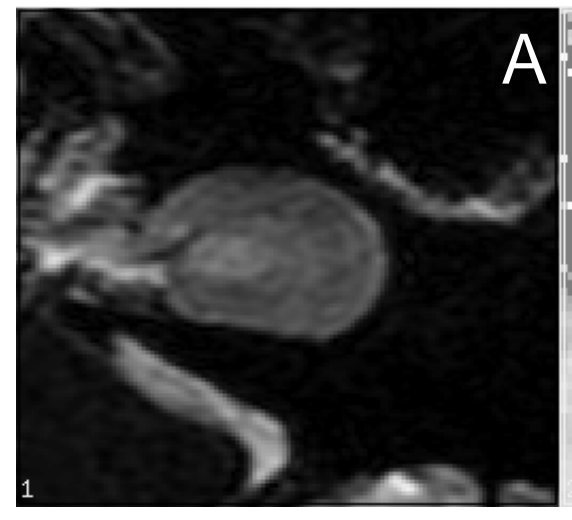

FSE image

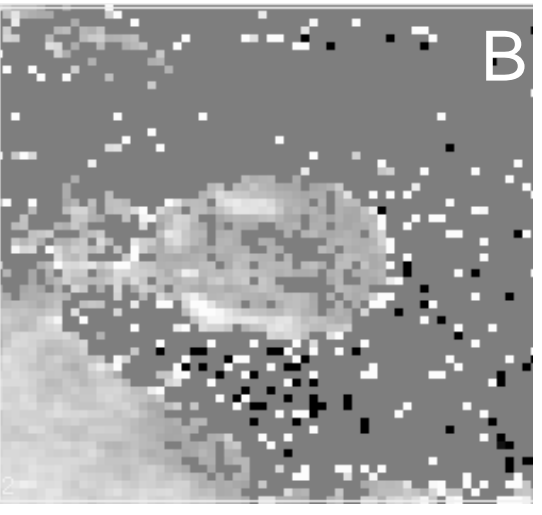

$\mathrm{T}_{2} *$ weighted image

Figure 4.20: $R O I$ selection for $T_{2}{ }^{*}$ calculations. 
The image data set from a diabetic animal (R03) is presented below.

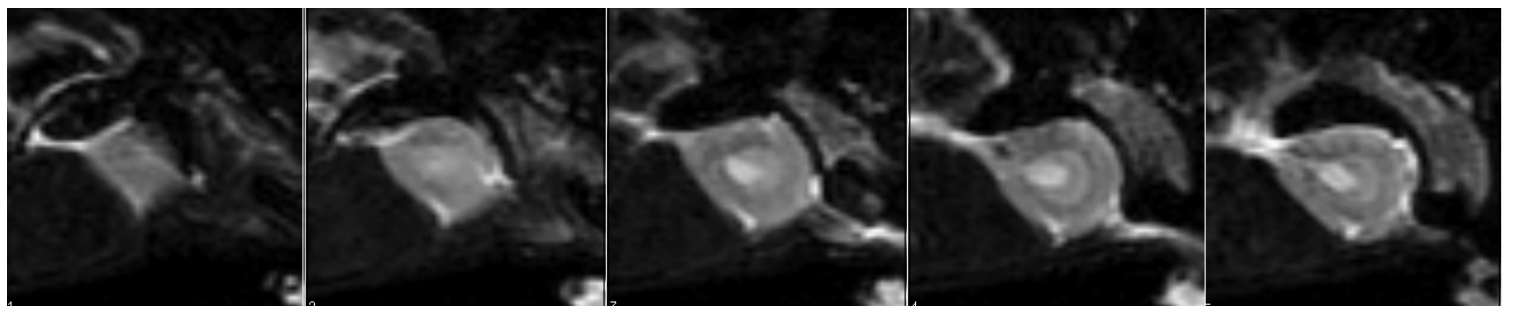

Figure 4.21: FSE images of 5 slices

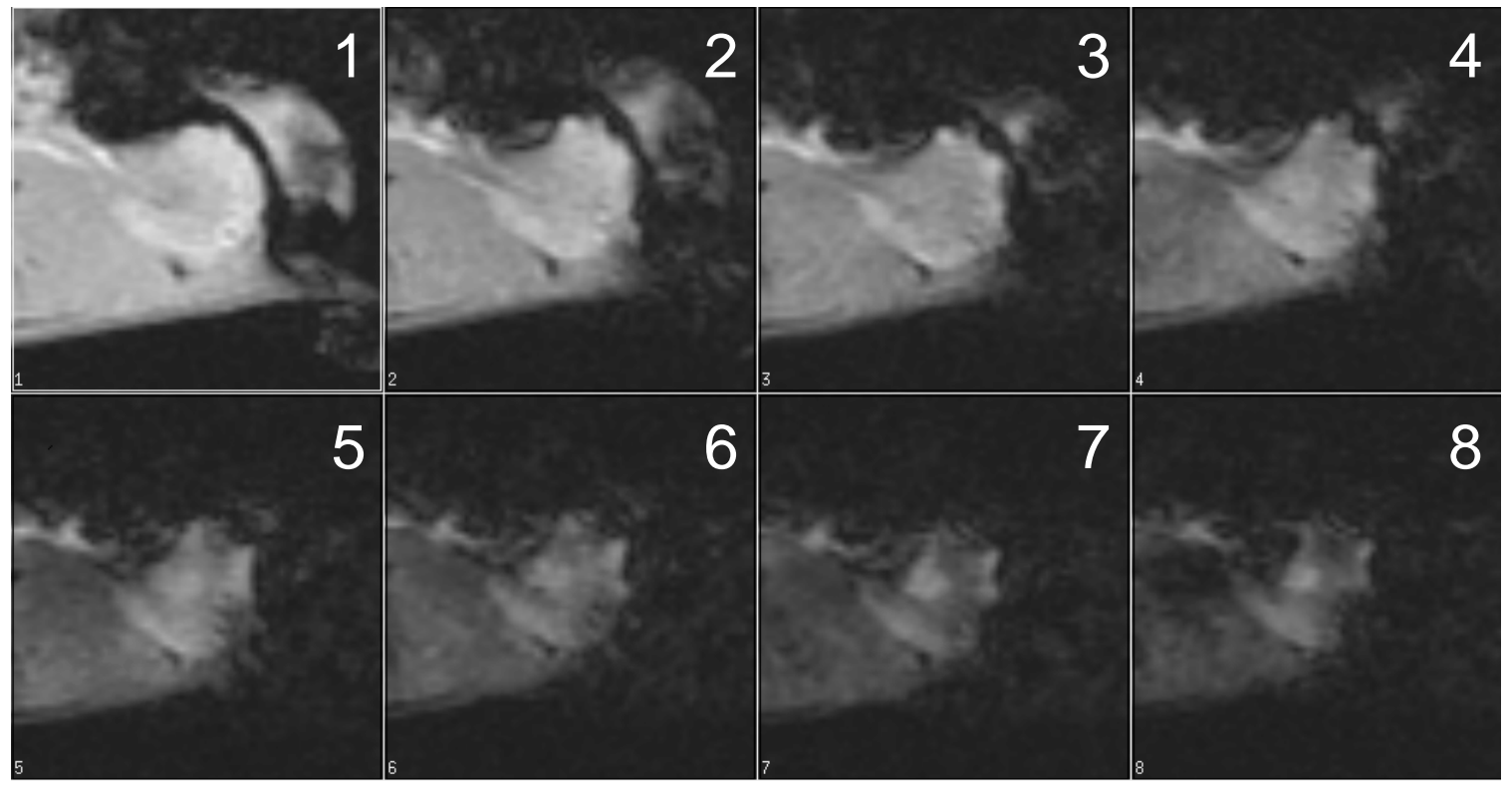

Figure 4.22: mGRE image set.

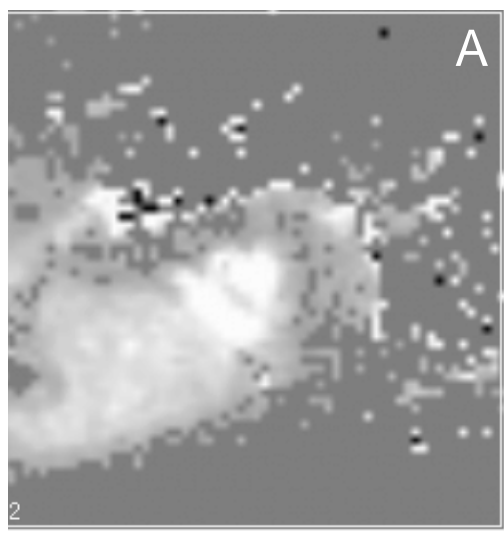

$\mathrm{T}_{2}{ }^{*}$ weighted image

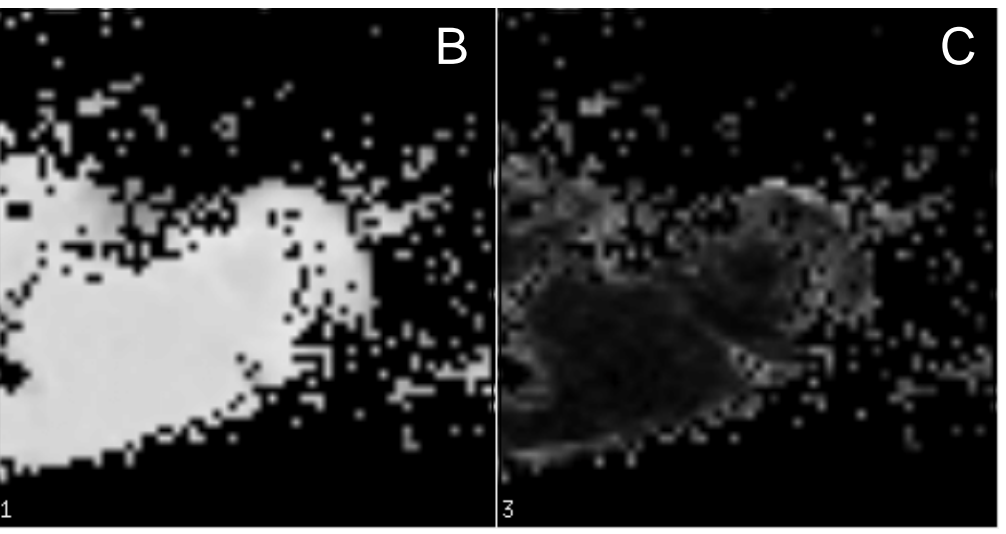

Intercept map

Figure 4.23: Post processed images. 


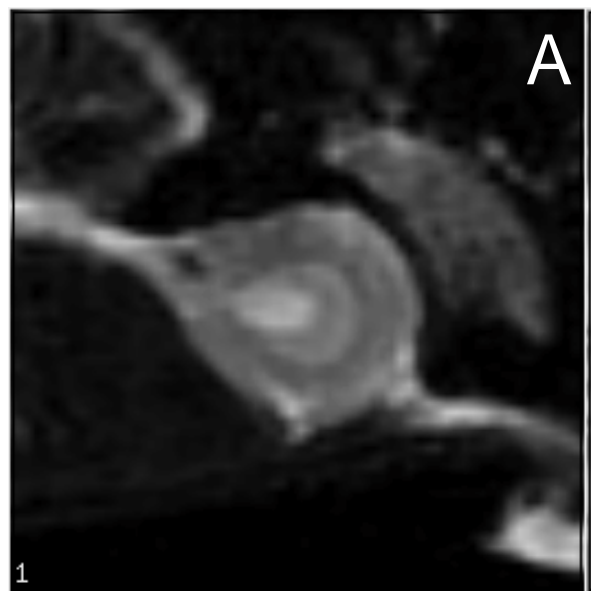

FSE image

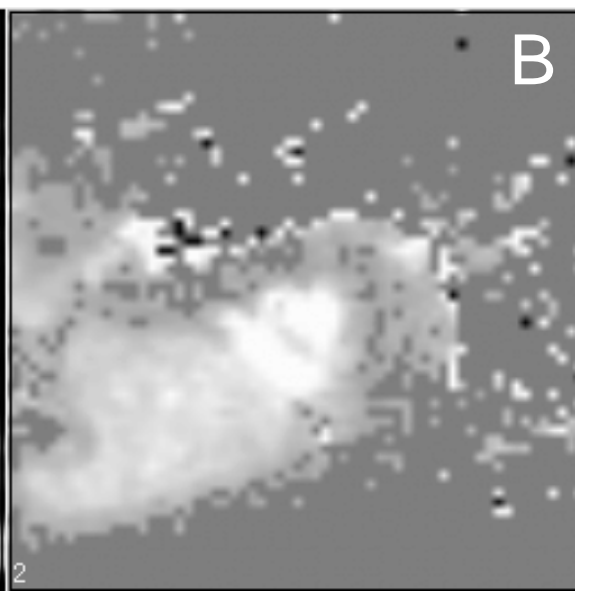

$\mathrm{T}_{2} *$ weighted image

Figure 4.24: ROI selection for $T_{2} *$ calculations.

\subsubsection{MRI-probe Tip Localization}

Locating the probe in the probe-MRI image was very crucial for understanding the values obtained from the $\mathrm{pO}_{2}$ and LDF. Even though the probe was inserted into the kidney at a calculated depth, due to the breathing of the animal, there was a slight probability for the probe to get displaced. During MRI scanning, the thin axial slices of SPGR images were used to locate the probe. The obtained MRI positional value was checked with the cross-sectional dissection of the kidney after scanning. The following were the locations for the animals imaged in this study:

Table 4.3: Probe location.

\begin{tabular}{|c|c|}
\hline Animal & Region of Probe tip \\
\hline Normal (R01) & Cortex \\
\hline Normal (R02) & Cortex \\
\hline Normal (R03) & Outer stripe (Medulla) \\
\hline Normal (R04) & Outer stripe (Medulla) \\
\hline Diabetic (R05) & Cortex \\
\hline Diabetic (R06) & Inner medulla \& papilla \\
\hline
\end{tabular}

The measurements are tabulated as shown below: 
Table 4.4: Oxygenation readings of both $M \mathrm{RI}$ and $\mathrm{pO}_{2}$ probe.

\begin{tabular}{|c|c|c|c|c|c|}
\hline Animal & Periods & $\begin{array}{c}\text { Anterior } \\
\text { Cortex } \\
\mathrm{T}_{2}^{*}(\mathrm{msec})\end{array}$ & $\begin{array}{c}\text { Posterior } \\
\text { Cortex } \\
\mathrm{T}_{2}{ }^{*}(\mathrm{msec})\end{array}$ & $\begin{array}{c}\text { Medulla } \\
\mathrm{T}_{2}{ }^{*}(\mathrm{msec})\end{array}$ & $\begin{array}{c}\text { Oxford } \\
\mathrm{pO}_{2} \\
\text { (mmHg) }\end{array}$ \\
\hline \multirow{4}{*}{$\begin{array}{c}\text { Normal } \\
\text { ( R01) }\end{array}$} & Ang & 161 & 223 & 121 & 820 \\
\hline & Ang-II & 16.4 & 22.3 & 13.1 & 83.9 \\
\hline & Control & 14.2 & 18.5 & 11.8 & 73.4 \\
\hline & Furosemide & 12.2 & 17.7 & 11.1 & 70 \\
\hline \multirow{3}{*}{$\begin{array}{c}\text { Normal } \\
\text { ( R02 ) }\end{array}$} & Ang-II & 16.4 & 24.3 & 22.5 & 59.2 \\
\hline & Control & 18.5 & 25.6 & 24.9 & 63.5 \\
\hline & Furosemide & - & - & - & - \\
\hline \multirow{4}{*}{$\begin{array}{c}\text { Normal } \\
\text { ( R03 ) }\end{array}$} & & 127 & 166 & 120 & 216 \\
\hline & Ang-II & 13.1 & 10.6 & 13.8 & $\angle 1.6$ \\
\hline & Control & 17.7 & 18.9 & 16.2 & 48.9 \\
\hline & Furosemide & 19.1 & 19.4 & 17 & 67.3 \\
\hline \multirow{3}{*}{$\begin{array}{c}\text { Normal } \\
\text { ( R04 ) }\end{array}$} & Ang-II & 7.5 & 10.9 & 17.5 & 65.3 \\
\hline & Control & 14.5 & 14.9 & 22.1 & 86.4 \\
\hline & Furosemide & 15.2 & 20.2 & 27.7 & 93.7 \\
\hline \multirow{3}{*}{$\begin{array}{c}\text { Diabetic } \\
\text { ( R05 ) }\end{array}$} & Ang-II & 10.8 & 23.8 & 13.2 & 65.2 \\
\hline & Control & 15.7 & 31.2 & 23.5 & 43.5 \\
\hline & Furosemide & - & - & - & - \\
\hline \multirow{3}{*}{$\begin{array}{c}\text { Diabetic } \\
\text { ( R06 ) }\end{array}$} & Ang-II & 19.2 & 24.8 & 20.5 & 13.5 \\
\hline & Control & 17.6 & 20.1 & 12.6 & 17.1 \\
\hline & Furosemide & - & - & - & - \\
\hline
\end{tabular}

Following were the inferences obtained from the obtained data set:

- The controls R02, R03 and R04 showed a consistency in the $\mathrm{pO}_{2}$ and $\mathrm{T}_{2} *$ trends. These values obtained from $\mathrm{pO}_{2}$ as well as $\mathrm{T}_{2}$ * were in good match with the previous studies $[13,19]$.

- For the control R01, the $\mathrm{T}_{2} *$ values obtained showed an increase with respect to the control, which was deviant from the previous studies [13]. The $\mathrm{pO}_{2}$ showed an increase with angiotensin II instead of the expected decrease.

Overall, this control animal responded opposite to the normal trend probably incorrect probe or procedure or due to an unwanted malfunctioning of the probe. 
- The diabetic animals $\mathrm{R} 05$ and $\mathrm{R} 06$, largely differed in both $\mathrm{pO}_{2}$ and $\mathrm{T}_{2} *$ values. In the case of R05, while Angiotensin II showed an increase in $\mathrm{pO}_{2}$, the $\mathrm{T}_{2}{ }^{*}$ values illustrated a decrease. This was the opposite in the case of R06. Here, while $\mathrm{pO}_{2}$ showed a decrease, the calculated $\mathrm{T}_{2} *$ represented an increase.

We suspect that the probes used in these two cases R01 \& R05 may have been out of calibration, which resulted in these abnormal values. Also, there is every possibility that due to induction of diabetes, the kidney functioning in R05 might be different.

The obtained values have been plotted with respect to the $\mathrm{pO}_{2}$ values obtained from the Oxford Optronix tissue oxygenation measurement system. Based on the location of probe tip, the $\mathrm{T}_{2}{ }^{*}$ values are correlated with the $\mathrm{pO}_{2}$ values obtained at that specific region.

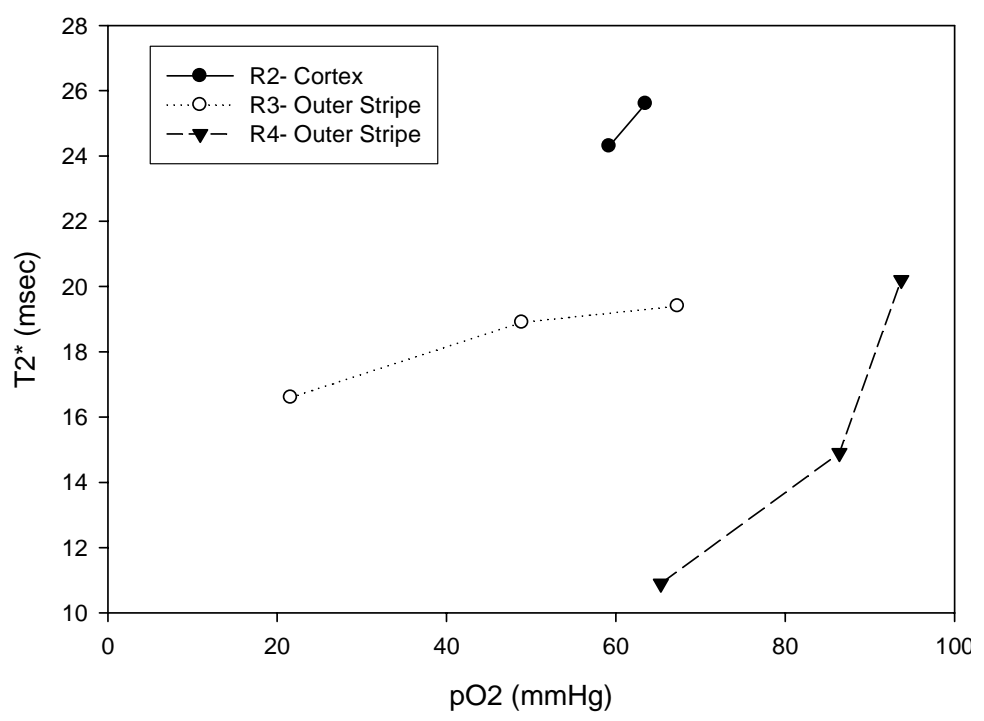

Figure 4.25: Plot indicating the correlation of $\mathrm{pO}_{2}$ with $\mathrm{T}_{2}{ }^{*}$ at the region of probe tip.

The plot indicates the correlation of $\mathrm{pO}_{2}$ invasive measurement with respect to the MRI measurements for specific regions at three different periods, Angiotensin-II, control and furosemide infusion respectively. 


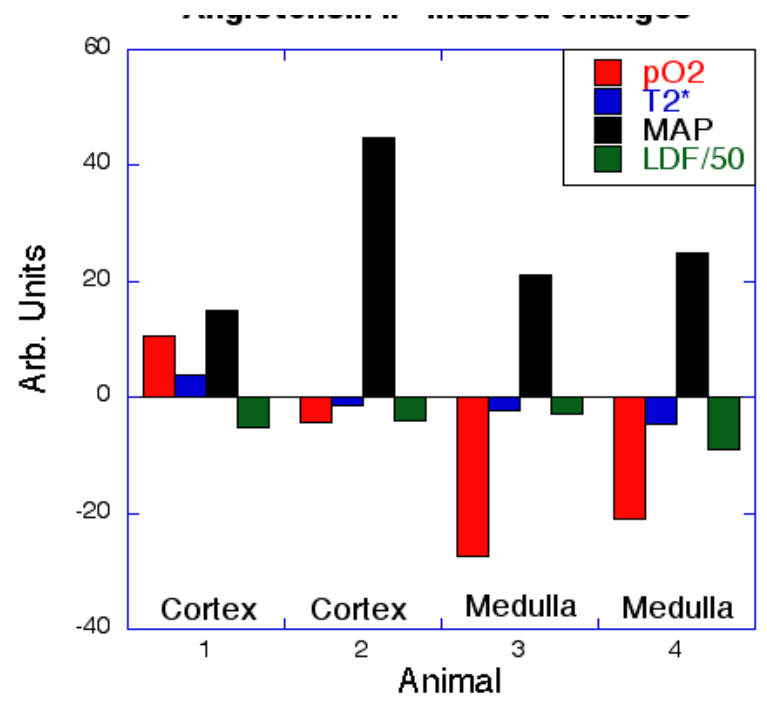

Angiotensin II - induced changes

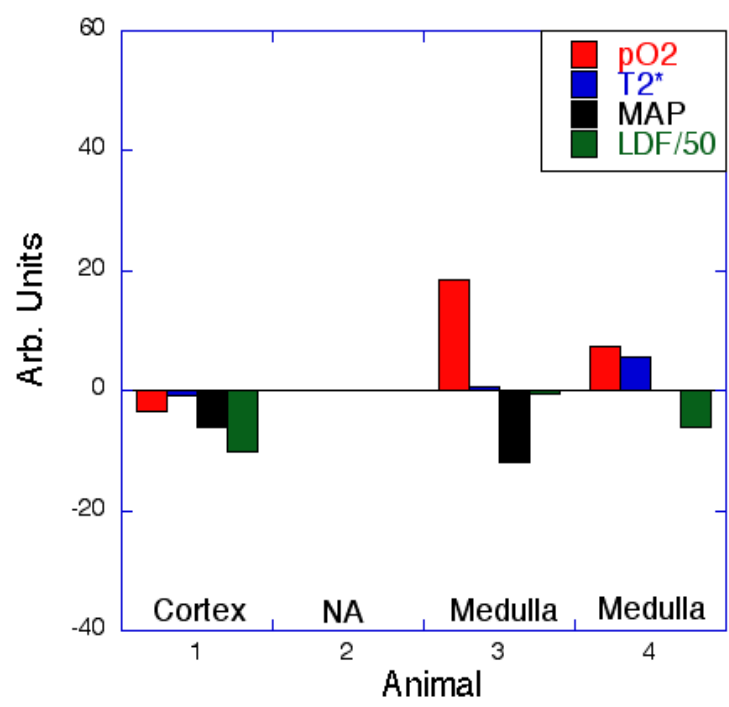

Furosemide - induced changes

Figure 4.26: Changes induced by drug infusion.

The histograms shown in figure 4.26, gives an approximate correlation of $\mathrm{pO}_{2}$, $\mathrm{T}_{2}{ }^{*}$, MAP and LDF. In animals 2, 3 and 4, we observe that $\mathrm{pO}_{2}, \mathrm{~T}_{2}{ }^{*}$ and LDF correlated well while the others had discrepancies.

\subsubsection{Perfusion Data Results}

The acquisition and processing steps, mentioned in section 3.3.2, were followed to obtain the perfusion-weighted images for the ASL data set. The problems encountered in the ASL data set of longitudinal study (section 4.2.2), were also observed here. The aliasing artifact and blurring were observed to be less relatively less when compared to that of the longitudinal ASL image set.

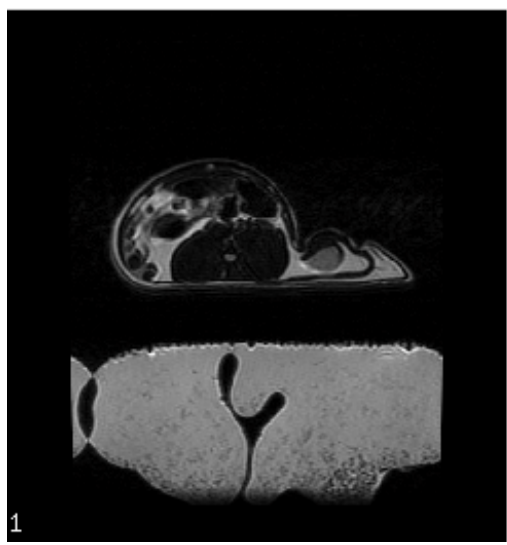

FSE image

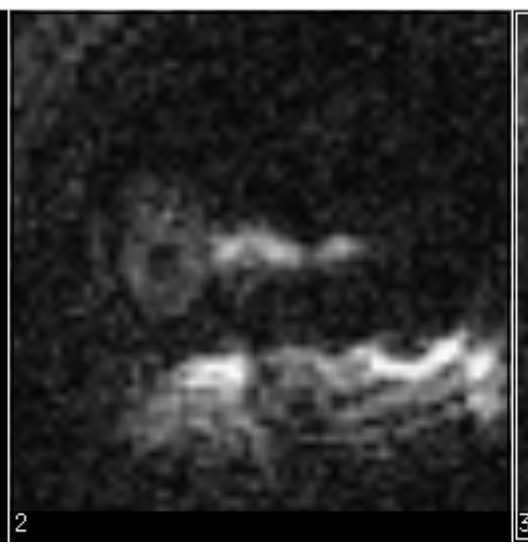

Tagged image

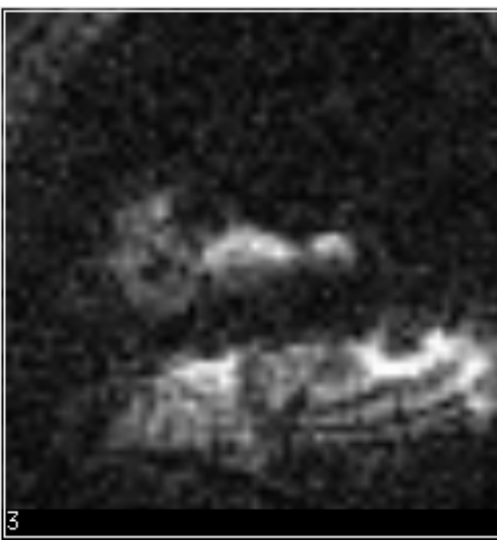

Control image

Figure 4.27: ASL data set from longitudinal study 


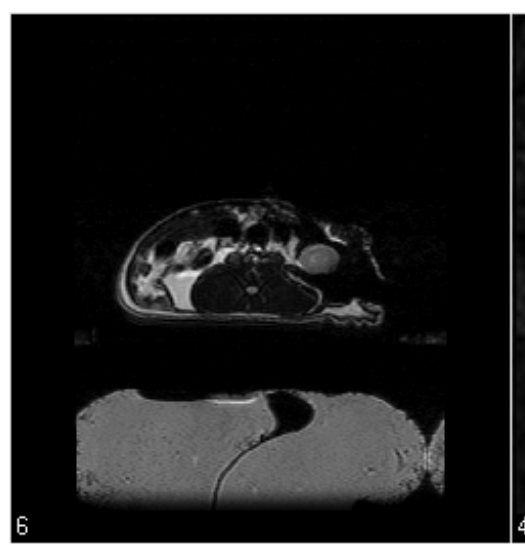

FSE image

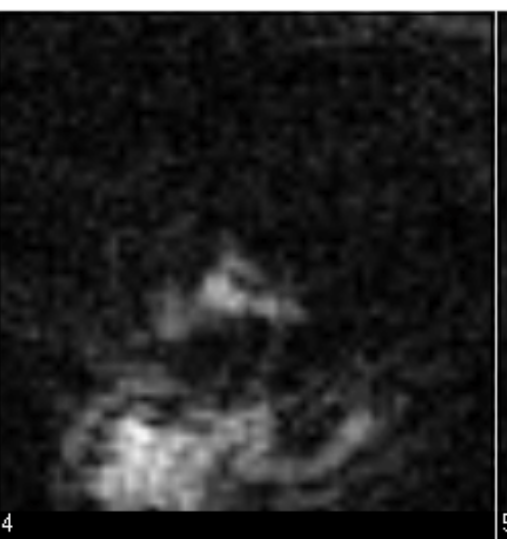

Tagged image

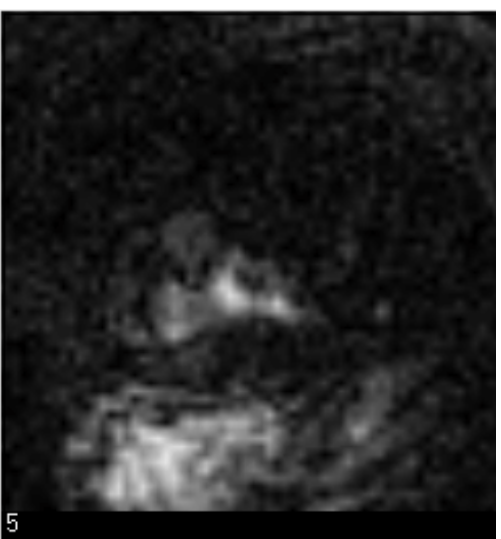

Control image

Figure 4.28: ASL data set from probe study

Apart from both blur and aliasing artifacts, the images from combined MRI-probe study (figure 4.28) had similar SNR when compared to the images from longitudinal study (figure 4.27).

$$
\begin{aligned}
& \mathrm{SNR}_{(\text {Longitudinal ASL image })}=\text { Mean }(\text { Signal }) / \text { Std. Dev. }(\text { Background })=6.15 \\
& \operatorname{SNR}_{(\text {MRI-Prove ASL image) }}=6.85
\end{aligned}
$$

Even though with little better SNR, perfusion measurements could not be obtained as the signal from the kidney was too low. The following two conditions were found to be dominant:

\section{Susceptibility from air:}

As the probe experiment involved a surgery, which exposed the kidney to the air, there was a considerable effect of the susceptibility artifact from atmospheric air. This led to the loss of signal at the anterior region of the kidney.

\section{Susceptibility from inserted probe:}

Also, there the combined oxygenation and blood flow probe inserted into the kidney, which led to a notable decrease in the SNR. 


\section{CHAPTER 5: DISCUSSION \& CONCLUSION}

\section{$\underline{5.1 \text { Discussion }}$}

\subsubsection{Longitudinal Study}

In this study, six animals were studied over a period of five months. The objective was to check $\mathrm{T}_{2} *$ stability over time. This study indicated that the $\mathrm{T}_{2}$ * values were stable over time in the posterior cortex $(97.8 \%)$ and medulla $(85 \%)$. The anterior cortex $\mathrm{T}_{2}{ }^{*}$ values were affected due to the kidney holder. An improved replacement for the kidney holder which can arrest the motion of the kidney without susceptibility artifact could lead measurement of accurate $\mathrm{T}_{2} *$ values in the anterior cortex.

Accuracy of these measurements was reasonably good as they match the values obtained in the previous studies [13]. We took measurements for the same ROI three times and found out that the $\mathrm{T}_{2}{ }^{*}$ values were within $5-8 \%$ of the published value.

\subsubsection{MRI-Probe Study}

We observed that mean arterial pressure was a good marker for indicating the change in kidney function. Correlation of $\mathrm{pO}_{2}$ and LDF to $\mathrm{T}_{2}{ }^{*}$ was observed in three animals. In the two animals with probes implanted in the medulla, the oxygenation went down with angiotensin II and up with furosemide in agreement with previously published results [34-36]. The blood flow response to these drugs, decrease with Angiotensin II and increase with furosemide, also agreed with previously published values [34-36] .

\section{Potential problems with imaging during MRI-Probe study}

Following are few issues addressed with respect to MR imaging:

\section{Signal Loss from susceptibility change from blood}

As describe in section 3.2.7, once the probe was inserted into the kidney, a gauze cloth wet with saline was placed around the kidney. This layer would keep the kidney outer surface from getting dried, which over time might change its physiology. 
In some images, where there was still bleeding, where gauze wet with blood came in direct contact with the kidney induced a noticeable signal loss in the mGRE images.

\section{Kidney Position}

To place the probe accurately in a pre-selected area of the kidney, it was important to immobilize the kidney. During scanning, a slight drift in the position of the kidney could change the probe placement (move it up or down) and alter the readings depending upon the region of the kidney. In order to overcome this problem, a pad made of silicon putty was placed beneath the kidney. Over time while exposed to body heat, the silicon pad deformed and the kidney began to sink slightly. This again changed the probe placement from its original position. Another solution to this problem was attempted. We replaced the silicon pad with clay, which unfortunately demonstrated a non-negligible susceptibility artifact during imaging.

\section{Susceptibility from air}

The anterior cortex $\mathrm{T}_{2}$ * values in the probe study varied due to the presence of air. The magnetic susceptibility of the air in addition to the presence of the plastic kidney holder caused a large relative signal loss (5-8\%) at lower TE's in the anterior cortex during the mGRE imaging. There was a $25-35 \%$ change when compared to the longitudinal $\mathrm{T}_{2} *$ values on the closed animals.

\section{Fat removal}

A layer of fat generally surrounds kidneys. Removal of this layer helped to visualize the kidney in the SPGR and FSE images. However, in the mGRE images, greater signal loss was observed over time (at later echoes) as the removal of fat lead to more exposure of the kidney to the air.

\section{Limitations of this experimental study}

1. As the probe experiments lasted for nearly three hours, longer in the early experiments while we were learning how to do the procedures, maintaining the animal physiology was a challenging task. Close monitoring and constant 
checking of its physiological parameters was very important. Any slight changes in the animal condition led to a noticeable change in the experimental readings and MR images. It was critically important that the animal remains stable when drugs are being administered to measure changes in kidney function, as kidney function is also dependent on cardiovascular and respiratory functions.

2. The probes used had a limited lifetime and were very delicate. The dye at the tip of the FQ probe is very sensitive and would lose its quenching effectiveness over time. Only one month operational guaranty was given by the manufacturer, although this has been very recently updated to 90 days. Re-use of the probe depends on its ability to detect dissolved oxygen in any liquid sample. A method to recheck the calibration would be very helpful but does not currently exist.

\section{$\underline{5.2 \text { Conclusion }}$}

At $3 \mathrm{~T}$ using mGRE, in rat kidneys, we observed that the $\mathrm{T}_{2}{ }^{*}$ values are stable to better than $10 \%$ when susceptibility effects can be neglected. The plastic holder provided good isolation from the intestines and held the kidney still. This is critical as the animals cannot hold their breath and ventilation or pacing would alter renal hemodynamics. The $\mathrm{T}_{2}$ * values are unaffected in the renal medulla and posterior cortex away from the cup.

The dual probe is MRI compatible and the measurements, while difficult, are accurate and as precise as probe placement allows. The use of two probes to measure values in both cortex and medulla, which is part of the future plan, may cause more problems with bleeding and susceptibility. Placement will be further complicated also.

At 3T, the increased signal to noise should have improved the ASL measurements but the increased susceptibility artifact overwhelmed any increased SNR. Smaller voxels will reduce this effect and improve image quality. The tagging pulse did not appear to be properly positioned and immediate work in the future must be undertaken to correct this 
problem. The tag thickness should also be tested. We would recommend that this work be accomplished in human brain first and then tested in the small animals (rats).

\subsection{Future Work \& Applications}

The mGRE pulse sequence needs no alteration with respect to the quality of images we acquire. Effective methods should be implemented to overcome the susceptibility artifacts from air and kidney holder. Also, better techniques to place and hold the probe at prescribed depths would make the investigator understand clearly the position of the probe in the kidney.

Currently, the ASL code suffers from poor image quality. Careful modifications to the pulse sequence are required to improve the image quality and give a better scope for good accurate measurements and repeatability.

It would useful to implant two probes would be used to get simultaneous reading from both cortex and medulla. This would give a better understanding of the functioning of both cortex and medulla at the same time.

At the time this thesis is being written, data from 4 control and 2 diabetic animals has been collected and analyzed. If the observed correlation of the MRI and the probe measurements continues with at least six more ( 2 control and 4 diabetic) animals, this method could be implemented successfully in a disease model. Future work would focus on a set of 4 diabetic and 2 control animals to study the disease progression and the renal physiological changes over time. Also, we anticipate that the probes would effectively be useful for validation in the diabetic model and would indicate a close correlation. 


\section{BIBLIOGRAPHY}

1. Baudelet, C. and B. Gallez, How does blood oxygen level-dependent (BOLD) contrast correlate with oxygen partial pressure (pO2) inside tumors? Magn Reson Med, 2002. 48(6): p. 980-6.

2. Wong, E.C., R.B. Buxton, and L.R. Frank, Quantitative perfusion imaging using arterial spin labeling. Neuroimaging Clin N Am, 1999. 9(2): p. 333-42.

3. http://users.ren.com/jkimball.ma.ultranet/BiologyPages/K/Kidney.html.

4. http://www.uhmc.sunysb.edu/internalmed/nephro/webpages/Part_A.htm.

5. http://academic.sun.ac.za/med_physbio/med_physiology/dept/kidney.htm.

6. Zou, A.P. and A.W. Cowley, Jr., Reactive oxygen species and molecular regulation of renal oxygenation. Acta Physiol Scand, 2003. 179(3): p. 233-41.

7. Hostetter, T.H., Prevention of the development and progression of renal disease. $\mathrm{J}$ Am Soc Nephrol, 2003. 14(7 Suppl 2): p. S144-7.

8. Hostetter, T.H., Diabetic nephropathy. N Engl J Med, 1985. 312(10): p. 642-4.

9 .http://www.clevelandclinicmeded.com/diseasemanagement/nephrology/diabeticne phropathy/diabeticnephropathy.htm.

10. http://renux.dmed.ed.ac.uk/EdREN/EdRenINFObits/Diabetic nephLong.html.

11. Caramori, M.L., P. Fioretto, and M. Mauer, The need for early predictors of diabetic nephropathy risk: is albumin excretion rate sufficient? Diabetes, 2000. 49(9): p. 1399-408.

12. O'Bryan, G.T. and T.H. Hostetter, The renal hemodynamic basis of diabetic nephropathy. Semin Nephrol, 1997. 17(2): p. 93-100.

13. Ries, M., et al., Renal diffusion and BOLD MRI in experimental diabetic nephropathy. Blood oxygen level-dependent. J Magn Reson Imaging, 2003. 17(1): p. 104-13.

14. Ogawa, Brain magnetic resonance imaging with contrast dependent on blood oxygenation. Proc Natl Acad Sci U S A, 1990. 87(24): p. 9868-72.

15. Juillard, L., et al., Blood oxygen level-dependent measurement of acute intrarenal ischemia. Kidney Int, 2004. 65(3): p. 944-50. 
16. Luh, W.-M., et al., Comparison of Simultaneously Measured Perfusion and BOLD Signal Increases During Brain Activation With T1-Based Tissue Identification. Magn. Res. Med., 2000. 44(1): p. 137-143.

17. Williams, D.S., et al., Perfusion imaging of the rat kidney with MR. Radiology, 1994. 190(3): p. 813-8.

18. Prasad, P.V., et al., Breath-hold R2* mapping with a multiple gradient-recalled echo sequence: application to the evaluation of intrarenal oxygenation. J Magn Reson Imaging, 1997. 7(6): p. 1163-5.

19. Prasad, Noninvasive evaluation of intrarenal oxygenation with BOLD MRI. Circulation, 1996. 94(12): p. 3271-5.

20. Kuperman, Differentiating between T1 and T2* changes caused by gadopentetate dimeglumine in the kidney by using a double-echo dynamic MR imaging sequence. J Magn Reson Imaging, 1996. 6(5): p. 764-8.

21. Glover, G.H., et al., Decomposition of inflow and blood oxygen level-dependent (BOLD) effects with dual-echo spiral gradient-recalled echo (GRE) fMRI. Magn Reson Med, 1996. 35(3): p. 299-308.

22. Speck, Functional imaging by I0- and $\mathrm{T}^{*}$-parameter mapping using multi-image EPI. Magn Reson Med, 1998. 40(2): p. 243-8.

23. Karam, Arterial Spin Labelling: Denmark.

24. Karger, N., et al., Quantitation of renal perfusion using arterial spin labeling with FAIR-UFLARE. Magn Reson Imaging, 2000. 18(6): p. 641-7.

25. Agus, Evaluation of changes in intrarenal oxygenation in rats using multiple gradient-recalled echo (mGRE) sequence. J Magn Reson Imaging., 1999. 9(6): p. 842-6.

26. Li, L.P., et al., Evaluation of the reproducibility of intrarenal R2* and DeltaR2* measurements following administration of furosemide and during waterload. $\mathrm{J}$ Magn Reson Imaging, 2004. 19(5): p. 610-6.

27. Williams, D.S., et al., Magnetic resonance imaging of perfusion using spin inversion of arterial water. Proc Natl Acad Sci U S A, 1992. 89(1): p. 212-6. 
28. Wang, Perfusion quantitation in transplanted rat kidney by MRI with arterial spin labeling. Perfusion quantitation in transplanted rat kidney by MRI with arterial spin labeling, 1998. 53(6): p. 1783-91.

29. Baudelet, C. and B. Gallez, Effect of anesthesia on the signal intensity in tumors using BOLD-MRI: comparison with flow measurements by Laser Doppler flowmetry and oxygen measurements by luminescence-based probes. Magn Reson Imaging, 2004. 22(7): p. 905-12.

30. Yang, Y., et al., Simultaneous perfusion and BOLD imaging using reverse spiral scanning at 3T: characterization of functional contrast and susceptibility artifacts. Magn Reson Med, 2002. 48(2): p. 278-89.

31. http://www.oxford-optronix.com/tissmon/index.htm.

32. Allen, Questions \& Answers in Magnetic Resonance Imaging. 2nd ed. 1994: C.V. Mosby.

33. Hollenberg, N.K., et al., Glomerular hemodynamics and the renin-angiotensin system in patients with type 1 diabetes mellitus. KIdney International, 2003. 63: p. 172-178.

34. Brezis, M., Y. Agmon, and F.H. Epstein, Determinants of intrarenal oxygenation. I. Effects of diuretics. Am J Physiol, 1994. 267(6 Pt 2): p. F1059-62.

35. Nobes, M.S., et al., Effects of angiotensin on renal cortical and papillary blood flows measured by laser-Doppler flowmetry. Am J Physiol, 1991. 261(6 Pt 2): p. F998-1006.

36. Mattson, D.L., Importance of the renal medullary circulation in the control of sodium excretion and blood pressure. Am J Physiol Regul Integr Comp Physiol, 2003. 284(1): p. R13-27.

37. http://www.cis.rit.edu/htbooks/mri/inside.htm.

38. Bushberg, The Essential Physics of Medical Imaging. 1994: Williams \& Wilkins.

39. Vadim, Magnetic Resonance Imaging. 2000: Academic Press.

40. Sprawls, Magnetic Resonance Imaging: Principles, Methods and Techniques. 2000: Medical Physics Publishing. 


\section{APPENDIX}

\section{A.1 Fundamentals of MRI}

Magnetic resonance imaging (MRI) is a technique used primarily in medical imaging to produce high quality images of the human body. MRI is based on the principles of nuclear magnetic resonance (NMR), a spectroscopic technique used by scientists to obtain microscopic chemical and physical information about molecules [37]. In 1946, Felix Bloch and Edward Purcell independently discovered that a magnetically energized substance bombarded with RF pulses, emitted a "tune" similar to a tuning fork. They found that the nuclei of different atoms absorbed radio waves at different frequencies. In 1952, Bloch and Purcell received the Nobel Prize for their discovery of what was referred to as Nuclear Magnetic Resonance (NMR).

Magnetic resonance imaging is based on the absorption and emission of energy in the radio frequency range of the electromagnetic spectrum. The human body is primarily composed of fat and water, approximately comprised of $63 \%$ hydrogen atoms. Hydrogen nuclei exhibit the strongest NMR signal. Thus MRI most commonly uses the NMR signal from the hydrogen's single proton. Protons possess a property called spin, which can be thought as a magnetic dipole and causes the nucleus to produce an NMR signal. Hydrogen nuclei magnetic moments are randomly oriented in the absence of an external magnetic field and are considered to have a net magnetization of zero [38].

The net magnetic moment per unit volume known as the magnetization vector (M) is given by:

$$
M=M_{x} i+M_{y} j+M_{z} k
$$

where, $\mathrm{i}, \mathrm{j}, \mathrm{k}$ are unit vectors in $\mathrm{x}, \mathrm{y}, \mathrm{z}$ directions respectively and $\mathrm{Mx}, \mathrm{My}, \mathrm{Mz}$ are magnetization in the $\mathrm{x}, \mathrm{y}, \mathrm{z}$ plane respectively.

In the absence of an external magnetic field, the net magnetization vector $\mathrm{M}$ will be zero. When placed in a strong external magnetic field $\mathrm{B}_{0}$, the magnetic moments of the 
protons align themselves and precess along the magnetic flux lines [39]. The magnetic moments of the protons precess along the z-direction (in the direction of $\mathrm{B}_{\mathrm{o}}$ ).
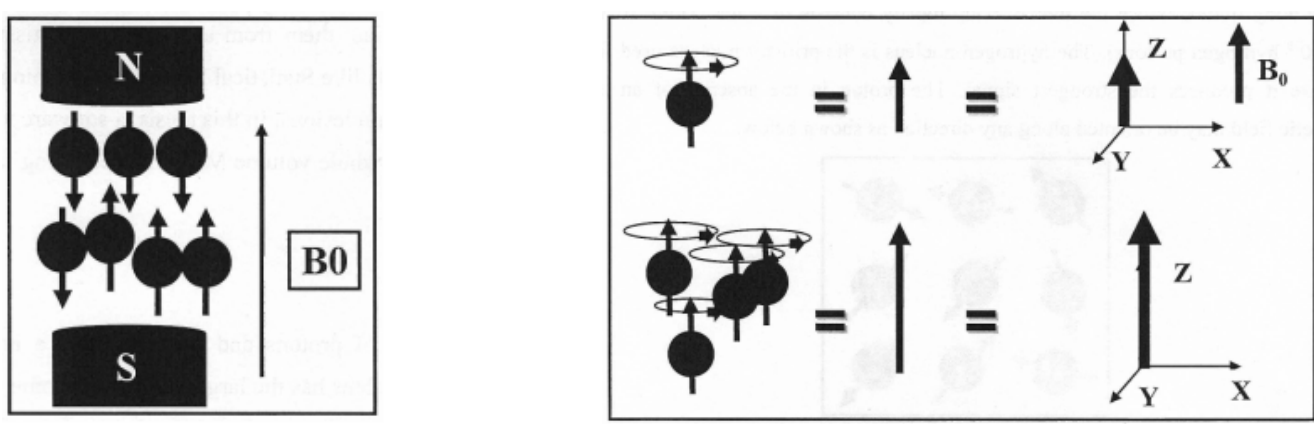

Figure A.1: Protons in a magnetic field aligned along the external magnetic field $B_{0}$. [37]

The equilibrium value of the magnitude of nuclear magnetization $\mathrm{M}_{0}$, (average of all individual nuclear magnetic dipole moments), in the presence of magnetic field $\mathrm{B}_{\mathrm{o}}$ is given as:

$$
M_{0}=\frac{N \gamma^{2} h^{2} I(I+1) B_{0}}{3 k T_{s}}
$$

Where, $\quad \mathrm{B}_{0} \quad-\quad$ Static magnetic field

$\begin{array}{lll}\mathrm{N} & - & \text { Number of nuclear spins per unit volume, } \\ \mathrm{G} & - & \text { Gyromagnetic ratio, a constant unique for each nucleus, } \\ \mathrm{H} & - & \text { Plank's constant, } \\ \mathrm{I} & - & \text { Nuclear spin, } \\ \mathrm{T}_{\mathrm{s}} & - & \text { Absolute sample temperature, } \\ \mathrm{K} & - & \text { Boltzmann's constant. }\end{array}$

The magnetization, $M_{0}$ is proportional to external magnetic field $B_{0}$. The magnetic moments exhibit the property of precessing around the field $\mathrm{B}_{\mathrm{o}}$. The frequency of precession is given by the Larmor frequency:

$$
f_{L}=\frac{\gamma B_{o}}{2 \pi}
$$


For the Hydrogen nucleus, $\frac{\gamma}{2 \pi}=42.58 \mathrm{MHz} /$ Tesla, thus the Larmor frequency will be in RF frequency region. The Larmor frequency is proportional to the magnetic field strength $\mathrm{B}_{\mathrm{o}}$ from equation (A1.3).

To obtain an MR signal, electromagnetic radiation (RF) $B_{1}$ is applied at the Larmor frequency $\left(f_{L}\right)$, perpendicular to the main magnetic field $\mathrm{B}_{\mathrm{o}}$, rotating the magnetization from its equilibrium position [39]. The protons are aligned along the $\mathrm{z}$-axis in equilibrium with the longitudinal magnetization $\left(\boldsymbol{M}_{z}\right)$ of magnitude $\boldsymbol{M}_{o}$. This alignment, when disturbed by a $90^{\circ}$ RF pulse displaces $\boldsymbol{M}_{z}$ to $\boldsymbol{M}_{x y}$.
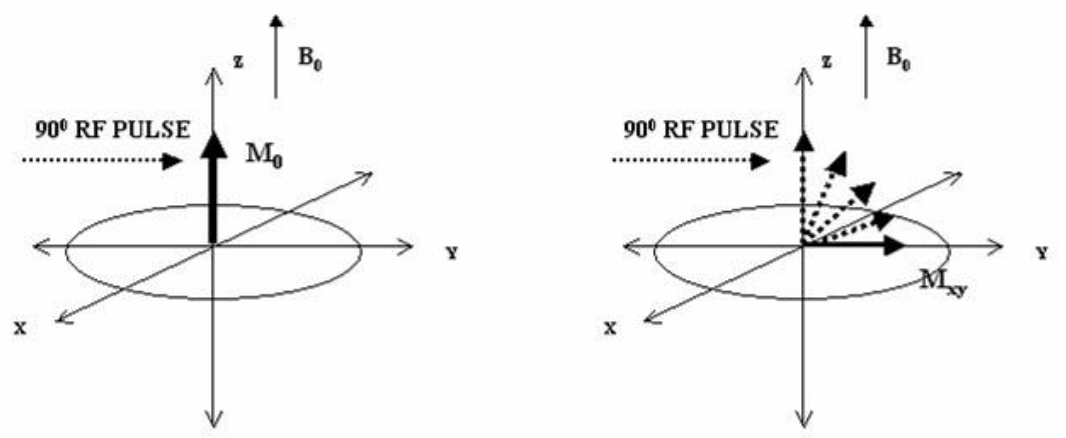

Figure A.2: Magnetization rotated by $90^{\circ}$ pulse.

The total displacement is proportional to the RF pulse energy and also the Larmor frequency. If the energy of the RF pulse is sufficient to tip the magnetization vector by $90^{\circ}$, then the $\boldsymbol{M}_{z}$ is tipped into the transverse plane $(\mathrm{x}, \mathrm{y})$. This time-varying magnetization induces flux changes, which are detected in a RF coil as MR signal.

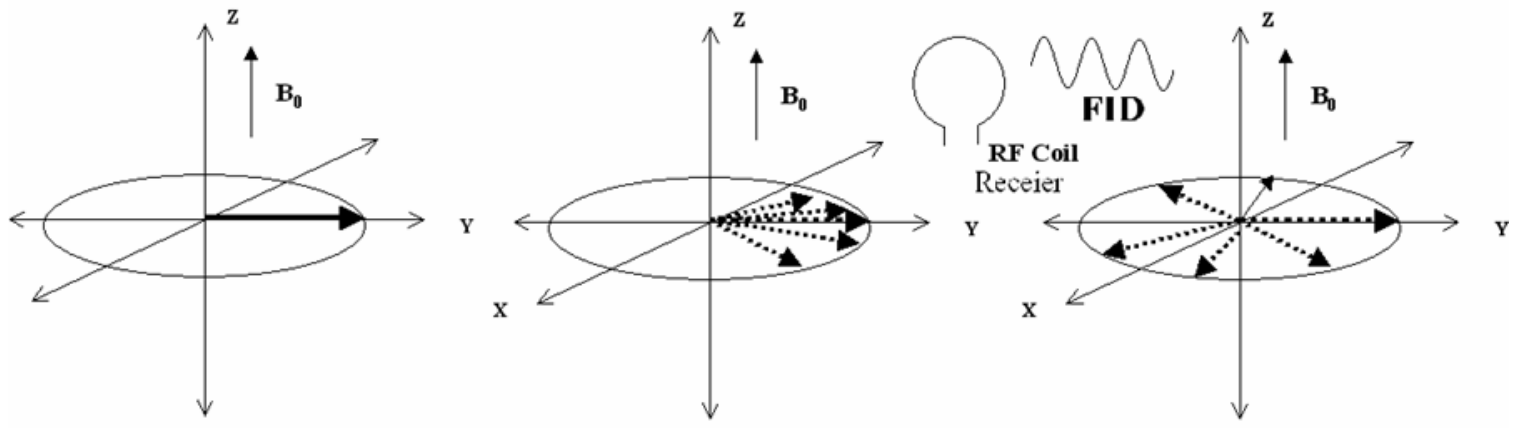

Figure A.3: Dephasing of transverse magnetization during free induction decay. 
The protons when returning to their equilibrium position along the $z$-axis undergo relaxation processes, which can be described in two ways:

1. The relaxation component of the net magnetization vector along the $z$-axis is called longitudinal or spin-lattice relaxation. The associated time constant is called $\mathrm{T}_{1}$.

2. The relaxation component of net magnetization vector in the $x y$ plane is called transverse or spin-spin relaxation and the associated time is called $\mathrm{T}_{2}$.

\section{A.1.1 Longitudinal Relaxation}

Longitudinal relaxation is the return of longitudinal magnetization to equilibrium $\left(\mathrm{B}_{0}\right)$ and is termed $\mathrm{T}_{1}$ recovery. As the hydrogen atoms release their energy they previously absorbed to the surrounding tissue (lattice), in their attempt to realign with the main magnetic field, $T_{1}$ relaxation or $T_{1}$ recovery occurs [38]. This phenomenon is sometimes referred to as "spin-lattice" relaxation and it occurs exponentially over time. That rate of recovery is a time constant and is referred to as $T_{1} . T_{1}$ recovery is the time it takes for $63 \%$ of the longitudinal magnetization to "regrow" or recover in the tissue. The equation governing this behavior as a function of the time ' $t$ ' is:

$$
M_{z}=M_{o}\left(1-\exp \left(\frac{-t}{T_{1}}\right)\right)
$$

Here, $M_{z}=$ Longitudinal Magnetization; $M_{o}=$ Equilibrium Magnetization

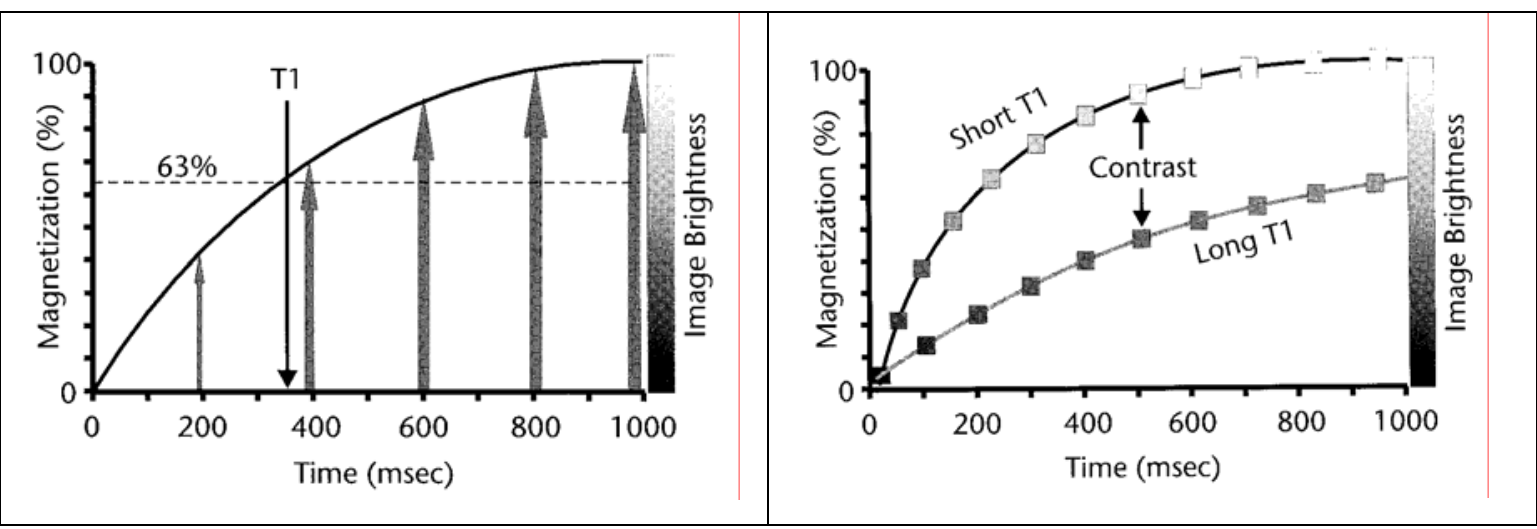

Figure A.4: Longitudinal magnetization recovery and tissue contrast. [40] 


\section{A.1.2 Transverse Relaxation}

Longitudinal magnetization recovery is accompanied by transverse relaxation. After an RF pulse, hydrogen nuclei spin in unison or in phase with each other. As the magnetic fields of all the nuclei interact with each other, energy is exchanged between those nuclei. The nuclei, which were spinning "in phase", lose their phase coherence or dephase with respect to each other. This process results in an exponential decrease in signal or decay in transverse magnetization and is known as $\mathrm{T}_{2}$ decay [32].

Due to the exchange of energy between spinning hydrogen nuclei, $\mathrm{T}_{2}$ decay is referred to as "spin-spin" relaxation. The rate of $\mathrm{T}_{2}$ decay is also expressed as a time constant. $T_{2}$ decay occurs when the transverse magnetization has decreased to $\mathbf{3 7 \%}$ of its initial value. The governing equation is given as follows:

$$
M_{X Y}=M_{X Y o} \exp \left(\frac{-t}{T_{2}}\right)
$$

Here, $M_{X Y}=$ Transverse Magnetization; $M_{X Y o}=$ Equilibrium Magnetization

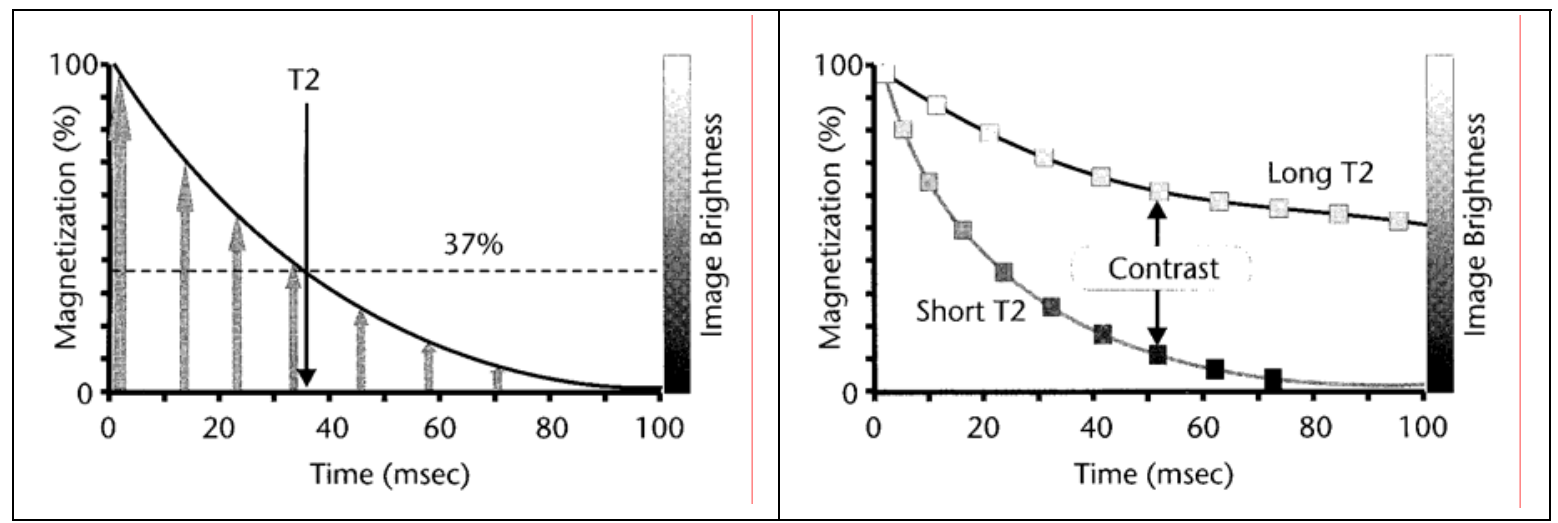

Figure A.5: Transverse magnetization decay and contrast. [40] 


\section{A.1.3 Tissue Contrast}

The difference in $T_{1}$ and $T_{2}$ relaxation constants allows differentiation between various tissues in the body. As well as $T_{1}$ - and $T_{2}$-contrast in tissues, proton density can also be measured. Proton density is a measure of number of protons per unit of tissue. Tissue contrast is affected by not only the $T_{1}$ and $T_{2}$ values of specific tissues, but the differences in the magnetic field strength, temperature changes and many other factors [32]. The user cannot control these parameters and instead obtains image contrast using various pulse sequences, with parameters such as bandwidth, flip angle, etc.

TR and TE are parameters commonly controlled by the operator that can be adjusted to vary image contrast for $\mathrm{T}_{1}$ and $\mathrm{T}_{2}$ and are usually measured in milliseconds.

- TR stands for repetition time, or the elapsed time between successive RF excitation pulses.

- TE stands for echo delay time, or the time interval between the RF pulse and the measurement of the first echo.

Generally, TR controls the $\mathrm{T}_{1}$-weighting of an MR image with a short $\mathrm{TR}$ maximizing $\mathrm{T}_{1}$-weighting. Also, $\mathrm{TE}$ controls the $\mathrm{T}_{2}$-weighting of an $\mathrm{MR}$ image with a short TE minimizing $\mathrm{T}_{2}$-weighting and a long TE maximizing $\mathrm{T}_{2}$-weighting [37].

\section{A.2 Localization of the MR signal}

During relaxation the protons trace a spiral motion, which can be descried by the Bloch equation [39]. The equation of motion of magnetization around the total magnetic field present is given by the Bloch equation as:

$$
\frac{d M}{d t}=\gamma M \times B
$$

It states that the time rate of change of change in $M$ is proportional to the cross product of $\mathrm{M}$ with $\gamma \mathrm{B}$. Hence if $\mathrm{B}$ is static, this motion corresponds to a precession of $\mathrm{M}$ 
about $\mathrm{B}$ at an angular frequency of $\gamma\|B\|$. If $\mathrm{B}$ is time varying, then the precessional frequency becomes time varying. The Bloch equation can be written to include the relaxation constants $T_{1}$ and $T_{2}$ and is given by:

$$
\frac{d M}{d t}=M \times \gamma B-\frac{\left(M_{x} i+M_{y} j\right)}{T_{2}}-\frac{\left(M_{z}-M_{o}\right) k}{T_{1}}
$$

The solution to the modified Bloch equation is shown below:

$$
\begin{aligned}
& M_{z}(t)=M_{o}\left(1-e^{-t / T_{1}}\right) \\
& M_{x}(t)=M_{x y} \cos w_{o} t\left(e^{-t / T_{2}}\right) \\
& M_{y}(t)=-M_{x y} \sin w_{o} t\left(e^{-t / T_{2}}\right)
\end{aligned}
$$

\section{A.2.1 Signal Measurement}

After the application of the RF pulses, emissions by the protons of RF radiation by the relaxation process at the resonant frequency take place [39]. Flux changes are detected in the receiver coil and the precessing magnetization in the transverse direction gives rise to an electromotive force $(\varepsilon)$ which is given as the rate of change of flux $(\varphi)$ in the coil.

$$
\varepsilon=-\frac{\partial \varphi}{\partial t}
$$

If $\mathrm{B}_{1}(x, y, z)$ is the magnetic field at a point $(x, y, z)$, the EMF in the coil is given as:

$$
d \varepsilon=-\frac{\partial}{\partial t}\left\{B_{1}(x, y, z) \bullet M(x, y, z, t)\right\} d r
$$

Thus the total EMF signal $s(t)$ is given as:

$$
\int_{\text {vol }} d \varepsilon=s(t)=-\int_{\text {vol }} \frac{\partial}{\partial t}\left\{B_{1}(x, y, z) \bullet M(x, y, z, t)\right\} d r
$$


The voltage is then amplified and demodulated to provide the MR signal.

\section{A.2.2 Signal Localization using Gradients}

Within the magnet, gradient coils are placed so that they superimpose their fields on the main magnetic field Bo. A set of gradient coils provides varying magnetic field strengths along the $x\left(G_{x}\right), y\left(G_{y}\right), z\left(G_{x}\right)$ direction. Gradients change only the spatial variation of the amplitude of $\mathrm{B}_{\mathrm{o}}$ but not the direction. Thus, the gradient coils in the three directions permit spatial information to be obtained [39]. Switching between the three gradients allows for image data signals to be obtained. Simplifying from equation (A 1.10) and adding gradient fields $G(t)$ in $x$ and $y$ direction, the received signal from an excited slice can be written as:

$$
s(t)=\int_{x} \int_{y} m(x, y) e^{-i 2 \pi k_{x}(t) x} e^{-i 2 \pi k_{y}(t) y} d x d y
$$

Thus the intensity of the signal is proportional to the magnitude of the magnetization. The above equation can be compared with 2-D Fourier transform of $\mathrm{m}$ (x, y) given as:

$$
M\left(k_{x}, k_{y}\right)=\int_{x} \int_{y} m(x, y) e^{-i 2 \pi\left(k_{x} x+k_{y} y\right)} d x d y
$$

From equations A 1.12 and $A 1.13$ we obtain the signal expression as:

$$
s(t)=M\left(\frac{\gamma}{2 \pi} \int_{0}^{t} G_{x}(\tau) d \tau, \frac{\gamma}{2 \pi} \int_{0}^{t} G_{y}(\tau) d \tau\right)=M\left(k_{x}, k_{y}\right)
$$

Thus at any time, the signal $s(t)$ equals the value of the $2 \mathrm{D}$ Fourier transform of $m(x, y)$. Thus the total signal obtained $s(t)$ is mapped to a trajectory through Fourier space. In MR raw data is stored in 2D Fourier space called " $k$ " space, where $k$ represents the spatial frequency variable. In order for the signal to be reconstructed, the trajectories of $s(t)$ need to cover a sufficient amount of $k$ space. The $k$ space data is then 2-D Fourier transformed to obtain the MR image. 


\section{A.3 MR Pulse Sequences}

Emphasizing the differences of spin density (the local hydrogen concentration) as well as $T_{1}$ and $T_{2}$ relaxation time constants of different tissues is the key to the exquisite contrast sensitivity of MR images. The pulse sequence refers to the sequence of RF gradient switching and RF detection used for MRI image acquisition. Pulse sequence physics exploits RF excitations to temporarily create spatially specific transverse magnetization during which magnetization frequency and phase are further manipulated by RF and gradient pulses[38]. RF and gradient pulses spatially encode the MR signal and give it properties dependent on the magnetic environment: proton density, $T_{1}, T_{2}$, $\mathrm{T}_{2}{ }^{*}$, chemical shift, flow effects, diffusion, magnetic susceptibility.

Tailoring the pulse sequences that is, the timing, order, polarity, and repetition frequency of the RF pulses and applied gradients can make the emitted signal dependent on theses relaxation characteristics. Present MR imaging technology relies on three major pulse sequence techniques: spin echo, inversion recovery, and gradient recalled echo sequences.

\section{A.3.1 Spin-Echo Pulse Sequence}

Spin echo is a very commonly used pulse sequence in MRI. It consists of a series of $90^{\circ}$ pulses separated by a period of TR (to allow total longitudinal recovery). Within the time period between $90^{\circ}$ pulses (TR), spin-spin interactions cause the resultant $\mathrm{M}_{\mathrm{xy}}$ signal to decay, according to the $\mathrm{T}_{2}$ time constant of the tissue (which is also influenced by the homogeneity of the magnet and therefore $\mathrm{T}_{2} *$ decay). After a time TE/2, a $180^{\circ} \mathrm{RF}$ pulse inverts the spin system and induces a rephasing of the transverse magnetization the formation of the echo at time TE [32]. Just before and after the peak amplitude of the echo, digital acquisition of the signal provides amplitude, phase and frequency information necessary for the image reconstruction process. 


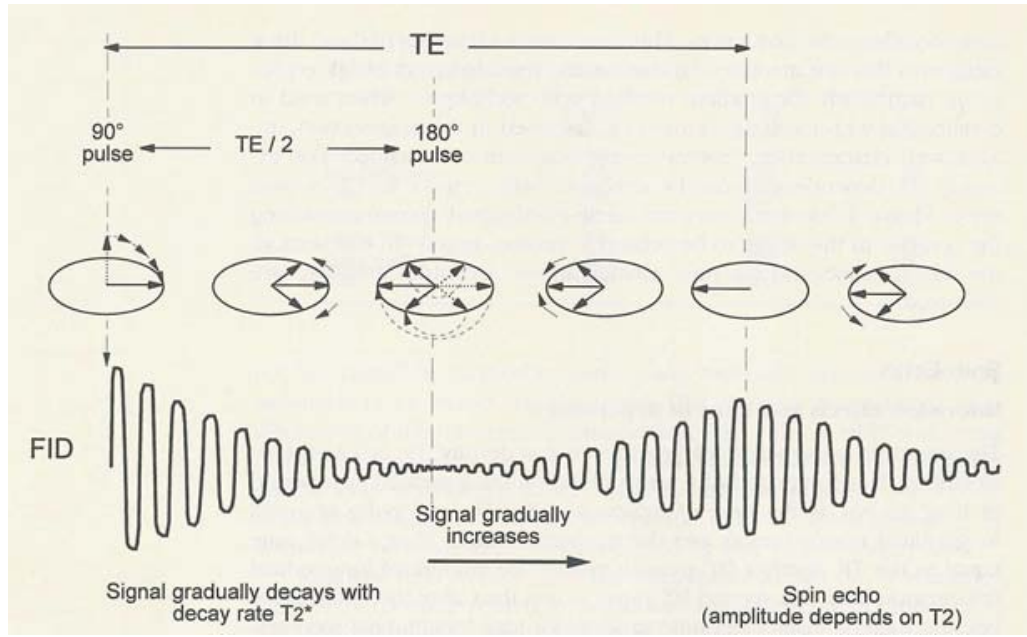

Figure A.6: The spin echo pulse sequence. [38]

Multiple echoes can be formed by repeated application of $180^{\circ}$ pulses of the spinecho pulse sequence. The elimination of extrinsic inhomogeneities gives the "true $\mathrm{T}_{2}$ " of the sample by fitting an exponential decay curve to the peak amplitude of the echoes, in the absence of gradients.

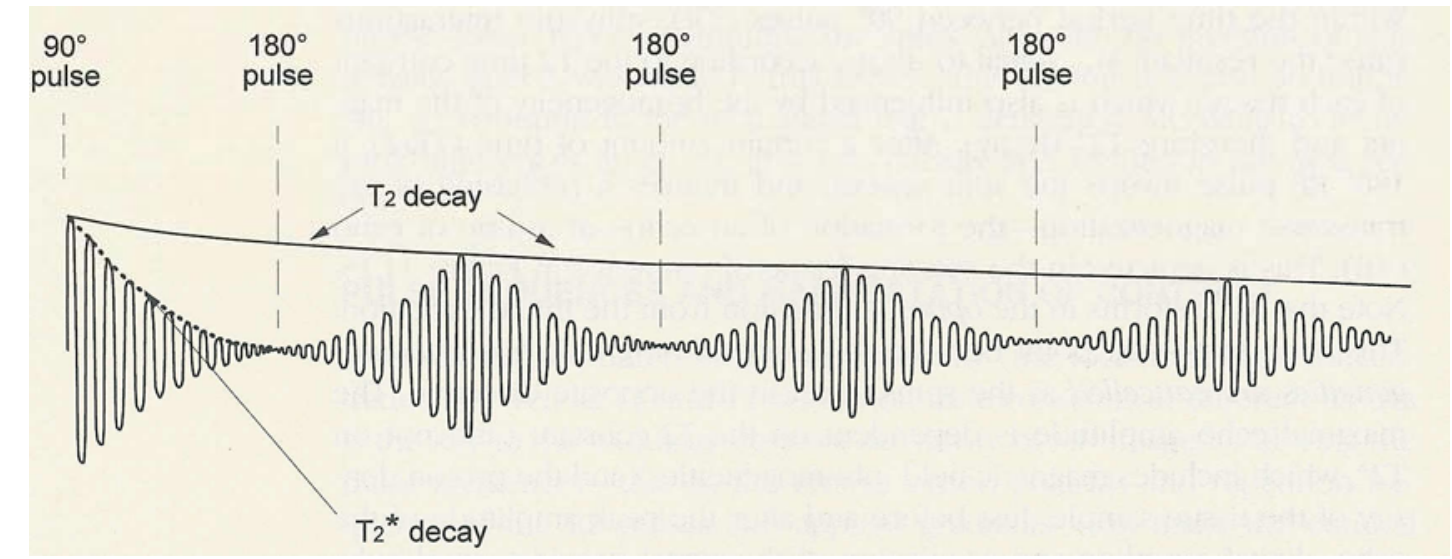

Figure A.7: $T_{2}$ decay [38]

The signal equation for a repeated spin echo sequence as a function of the repetition time, TR, and the echo time, TE, defined as the time between the $90^{\circ}$ pulse and the maximum amplitude in the echo is given as:

$$
S=k \rho\left(1-\exp \left(\frac{-T R}{T_{1}}\right)\right) \exp \left(\frac{-T E}{T_{2}}\right)
$$

Here $S$ is the amplitude of the signal after being Fourier transformed; $k$ is proportionality constant and $\rho$ is the density of spins in the sample. The timing diagram for a spin-echo 
imaging sequence has entries for the RF pulses, the gradients in the magnetic field, and the signal is listen in figure A.8.

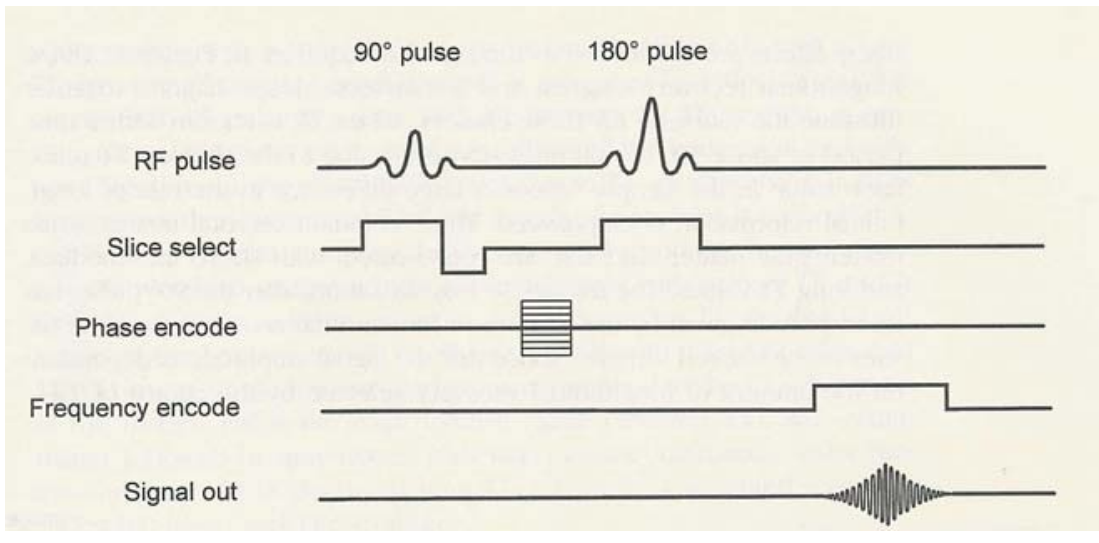

Figure A.8: A generic spin-echo pulse sequence diagram.[38]

A slice selective $90^{\circ}$ RF pulse is applied in conjunction with a slice selection gradient. At time equal to $\mathrm{TE} / 2$, a slice selective $180^{\circ}$ pulse is applied in conjunction with the slice selection gradient. A phase encoding gradient is applied between the $90^{\circ}$ and $180^{\circ}$ pulses. The phase encoding gradient is varied in 128 or 256 steps between $\mathrm{G}_{\phi_{\mathrm{m}}}$ and $\mathrm{G}_{\phi_{\mathrm{m}}}$. The frequency encoding gradient is applied after the $180^{\circ}$ pulse during the time in which the echo is collected. The recorded signal is the echo.

\section{A.3.2 Inversion Recovery (IR)}

In this sequence, an $180^{\circ}$ pulse is first applied, which inverts the net magnetization down to the $-\mathrm{Z}$ axis. The magnetization undergoes spin-lattice relaxation and returns toward its equilibrium position along the $+Z$ axis. Once magnetization is present in the $\mathrm{XY}$ plane it rotates about the $\mathrm{Z}$ axis giving a FID. The signal (S) as a function of TI when the sequence is not repeated is given below:

$$
S=k \rho\left(1-2 \exp \left(\frac{-T I}{T_{1}}\right)\right)
$$

It should be noted at this time that the zero crossing of this function occurs for TI $=\mathrm{T}_{1} \ln 2$. When an inversion recovery sequence is repeated every TR seconds, for signal averaging or imaging purposes, the signal equation becomes: 


$$
S=k \rho\left(1-2 \exp \left(\frac{-T I}{T_{1}}\right)+\exp \left(\frac{-T R}{T_{1}}\right)\right)
$$

\section{A.3.3 Gradient Recalled Echo (GRE)}

GRE uses a magnetic field gradient to induce echo formation. The gradient causes a dispersion of the frequencies of the protons under its influence. In the case of a FID signal generated after a $90 \mathrm{RF}$ pulse, the coherent transverse magnetization will rapidly be dephased. By reversing the polarity of the same gradient, the spins will be rephased at a time when the gradient field strength is balanced (equal negative and positive gradient amplitudes), resulting in a gradient echo. To complete the echo, the later gradient remains for twice the time as the initial dephasing gradient.

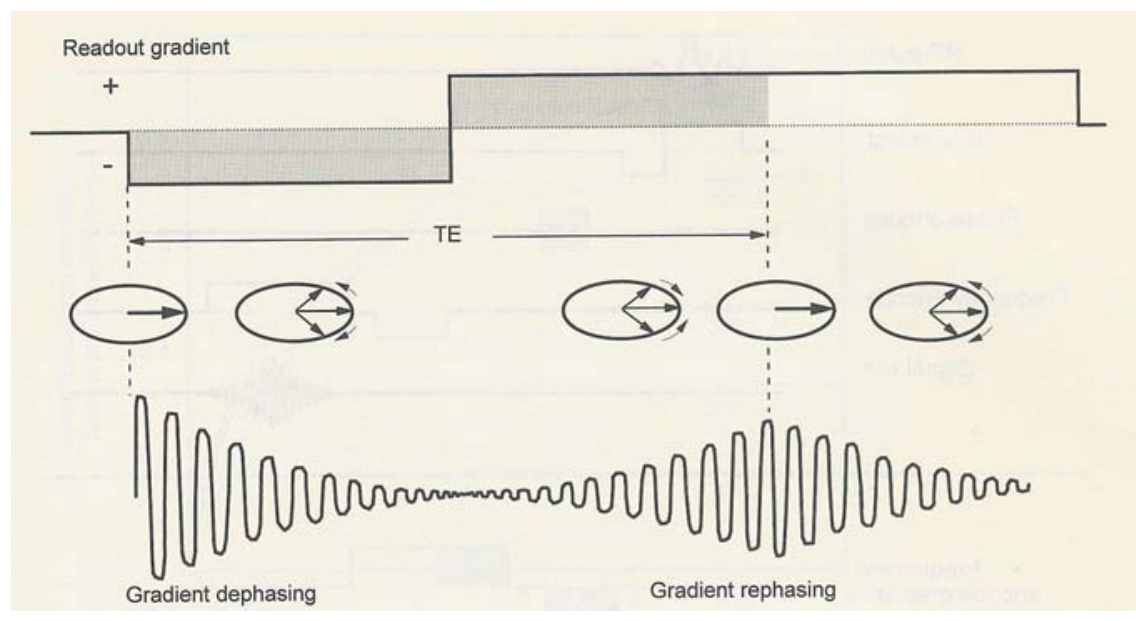

Figure A.9: The gradient echo sequence.[38]

Gradient echoes are very sensitive to magnetic field imperfections, whether arising from external magnetic field itself or from variations caused by magnetic susceptibility of the tissues. The decay of the transverse magnetization is therefore a strong function of $\mathrm{T}_{2}{ }^{*}$. A major variable determining tissue contrast in the GRE sequence is the flip angle. Depending on desired contrast, the flip angle can range from a few degrees to $90^{\circ}$. A larger flip angle will generate more transverse magnetization for a short TR than will the smaller flip angle, due to the steady-state magnetization that quickly builds up in the sample. 